МатематиЧеСКИЙ ИНСтитУт им. В. А. СтекЛовА РОССИЙСКОЙ АКАДЕМИИ НАУК

\title{
Современные проблемы математики
}

\section{Bыnуск 6}

Издание выходит с 2003 года

\section{С. П. Суетин}

Сравнительная асимптотика решений и формулы следов для некоторого класса разностных уравнений

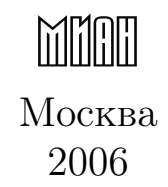


УДК $517.53+517.984+517.962$

ББК (B)22.161.5

C89

\section{Редакционный совет:}

С.И. Адян, Д.В. Аносов, О.В. Бесов, В.С. Владимиров,

А.М. Зубков, А.Д. Изаак (ответственный секретаръ),

А.А. Карачуба, В. В. Козлов, С.П. Новиков, С.П. Коновалов, А.Н. Париин (заместитель главного редактора), Ю.В. Прохоров, А.Г. Сергеев (главный редактор), А.А. Славнов, Д.В. Трещев, Е. М. Чирка

C89 Современные проблемы математики / Математический институт им. В. А. Стеклова РАН (МИАН). - М.: МИАН, 2006. Вып. 6: Сравнительная асимптотика решений и формулы следов для некоторого класса разностных уравнений / Суетин С. П. - 72 с.

ISBN 5-98419-015-X

Серия "Современные проблемы математики" - рецензируемое продолжающееся издание Математического института им. В. А. Стеклова PAН. В серии публикуются работы, отражающие научные достижения сотрудников и аспирантов МИАН. Особое внимание уделяется исследованиям, выполненным в рамках научных программ Российской академии наук. Публикация работ осуществляется по решению Редакционного совета, в который входят представители администрации и заведующие отделами МИАН. Издания серии рассылаются по стандартному обязательному списку, в библиотеки математических институтов и ведущих университетов страны.

ISBN 5-98419-015-X

(c) Математический институт им. В. А. Стеклова РАН, 2006 


\section{Оглавление}

$\S 1$ Введение . . . . . . . . . . . . . . . . 5

$\S 2$ Формулировка основных результатов . . . . . . . . . . . 18

§ 3. Доказательство теоремы 1 . . . . . . . . . . . . . . . . . . . 24

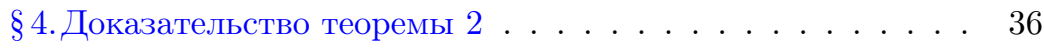

Приложение А . . . . . . . . . . . . . . . . . . . . . . . . . 40

Приложение В. Доказательство существования и вывод явных формул для $\Psi$-функции . . . . . . . . . . . . . . . . . . . . 48

Приложение C . . . . . . . . . . . . . . . . . . . . . . . . . 69

Список литературы . . . . . . . . . . . . . . . . . . 72 



\section{§1. Введение}

1. Пусть $\mu$ - положительная борелевская мера с компактным носителем supp $\mu$ на вещественной прямой $\mathbb{R}$,

$$
\widehat{\mu}(\lambda):=\int \frac{d \mu(x)}{\lambda-x}, \quad \lambda \in \widehat{\mathbb{C}} \backslash \operatorname{supp} \mu,
$$

- марковская функиия, соответствующая мере $\mu$. Всюду в дальнейшем мы будем считать меру $\mu$ единичной: $|\mu|=1$. В [1] П. Л. Чебышёв с помощью классического алгоритма Евклида сопоставил функции $\widehat{\mu}$ чебъшевскую непреръьнуюю дробъ (часто называемую теперь $J$-дробью):

$$
\begin{aligned}
\widehat{\mu}(\lambda) & =\frac{1}{\lambda-b_{1}-f_{1}(\lambda)}=\frac{1}{\lambda-b_{1}-\frac{a_{1}^{2}}{\lambda-b_{2}-f_{2}(\lambda)}} \\
& \frac{1}{\lambda-b_{1}-\frac{a_{1}^{2}}{\lambda-b_{2}-\frac{a_{2}^{2}}{\ddots}}}
\end{aligned}
$$

где все $a_{n}, b_{n}$ - вещественны, $a_{n} \neq 0$ и

$$
\sup _{n}\left|a_{n}\right|<\infty, \quad \sup _{n}\left|b_{n}\right|<\infty .
$$

Коэффициенты $a_{n}$ и $b_{n}$ строятся непосредственно по коэффициентам разложения функции $\widehat{\mu}$ в ряд Лорана в бесконечно удаленной точке $\lambda=\infty$

$$
\widehat{\mu}(\lambda)=\sum_{n=0}^{\infty} \frac{s_{n}}{\lambda^{n+1}},
$$

где

$$
s_{n}=\int x^{n} d \mu(x), \quad n=0,1,2, \ldots,
$$

Работа выполнена при поддержке РФФИ (грант № 05-01-01027), INTAS (грант № 03-51-6637) и Программы поддержки ведущих научных школ РФ (грант № НШ-4466.2006.1). 
- моменты меры $\mu$. В дальнейшем будем считать, что все $a_{n}>0$. Хорошо известно, что $n$-я подходящая дробь

$$
\frac{P_{n}}{Q_{n}}(\lambda):=\frac{1}{\lambda-b_{1}-\frac{a_{1}^{2}}{\lambda-b_{2}-\frac{a_{2}^{2}}{\lambda-b_{n-1}-\frac{a_{n-1}^{2}}{\lambda-b_{n}}}}}
$$

к непрерывной дроби (2) обладает следующим характеристическим свойством

$$
\frac{P_{n}}{Q_{n}}(\lambda)=\frac{s_{0}}{\lambda}+\frac{s_{1}}{\lambda^{2}}+\cdots+\frac{s_{2 n-1}}{\lambda^{2 n}}+O\left(\frac{1}{\lambda^{2 n+1}}\right), \quad \lambda \rightarrow \infty
$$

тем самым,

$$
\widehat{\mu}(\lambda)-\frac{P_{n}}{Q_{n}}(\lambda)=O\left(\frac{1}{\lambda^{2 n+1}}\right), \quad \lambda \rightarrow \infty .
$$

Многочлены $Q_{n}$ имеют степень ровно $n$, ортогоналъны по мере $\mu$

$$
\int Q_{n}(\lambda) \lambda^{k} d \mu(\lambda)=0, \quad k=0,1, \ldots, n-1,
$$

нормировкой $Q_{n}(\lambda)=\lambda^{n}+\cdots$ определены однозначно и удовлетворяют следующим трехчленным рекуррентным соотношениям:

$$
\begin{aligned}
& Q_{n}(\lambda)=\left(\lambda-b_{n}\right) Q_{n-1}(\lambda)-a_{n-1}^{2} Q_{n-2}(\lambda), \\
& Q_{0}(\lambda) \equiv 1, \quad Q_{1}(\lambda)=\lambda-b_{1} .
\end{aligned}
$$

Многочлены $P_{n}, \operatorname{deg} P_{n}=n-1$, определяются непосредственно по $Q_{n}$ :

$$
P_{n}(\lambda)=\int \frac{Q_{n}(\lambda)-Q_{n}(x)}{\lambda-x} d \mu(x)
$$

и называются многочленами второго рода, функция

$$
R_{n}(\lambda):=\int \frac{Q_{n}(x) d \mu(x)}{\lambda-x}, \quad \lambda \in \widehat{\mathbb{C}} \backslash \operatorname{supp} \mu,
$$

в теории ортогональных многочленов называется функиией второго рода. Все три функции $Q_{n}, P_{n}$ и $R_{n}$ связаны очевидным соотношением:

$$
\left(Q_{n} \widehat{\mu}-P_{n}\right)(\lambda)=R_{n}(\lambda)
$$


при этом (ср. с (3))

$$
R_{n}(\lambda)=\frac{1}{Q_{n}(\lambda)} \int \frac{Q_{n}^{2}(x) d \mu(x)}{\lambda-x}=O\left(\frac{1}{\lambda^{n+1}}\right), \quad \lambda \rightarrow \infty
$$

Все вышеуказанные соотношения ${ }^{1}$ в достаточно ясной форме содержатся в [1] для случая общих ортогональных многочленов (см. также [2]). Другое дело, что работа [1] была написана Чебышёвым задолго до знаменитых работ Стилтьеса 1894-1895 гг. (см. [3], а также [2]), где впервые появился интеграл Стилтьеса, причем тоже в связи с изучением непрерывных дробей. Поэтому в [1] Чебышёв имеет дело с функциями вида ${ }^{2}$

$$
f(\lambda)=\sum_{j=1}^{N} \frac{\theta_{j}^{2}}{\lambda-x_{j}}, \quad \lambda \neq x_{j}
$$

где $N<\infty$. Соответствующая непрерывная дробь (2) оказывается конечной, а ортогональные многочлены $Q_{k}$ - заданными при $k=0,1, \ldots, N$. Но для справедливости соотношений типа $(3)-(7)$ это не имеет значения. Самому Чебышёву была ясна общность полученных им в [1] результатов. Так, в [4], рассматривая функции вида

$$
\frac{1}{\pi} \int_{-1}^{1} \frac{1}{\lambda-x} \frac{d x}{\sqrt{1-x^{2}}}, \quad \sqrt{\frac{k}{\pi}} \int_{-\infty}^{\infty} \frac{e^{-k x^{2}}}{\lambda-x} d x, \quad k \int_{0}^{\infty} \frac{e^{-k x}}{\lambda-x} d x
$$

при $k>0$, он не повторял заново рассуждений [1], а пользовался уже сделанными там выводами. В связи с первой функцией (8) Чебышёв вывел явную формулу для классических многочленов Чебышёва, ортогональных на отрезке $[-1,1]$ по мере $d \mu(x)=\frac{1}{\pi}\left(1-x^{2}\right)^{-1 / 2} d x$. В дальнейшем он доказал, что именно они дают решение задачи о многочлене с фиксированным старшим коэффициентом, наименее уклоняющемся от нуля в равномерной норме на $[-1,1]$. В связи с разложением в непрерывную дробь второй и третьей функций (8) Чебышёв в 1860 году в [4] привел формулы и для двух других классических ортогональных

\footnotetext{
${ }^{1}$ А кроме них - формула Кристоффеля-Дарбу и полиномиальное воспроизводящее ядро Сегё.

${ }^{2}$ Подробнее см. приложение С.
} 
многочленов, называемых теперь (см., например, [2], [5]) соответственно многочленами Чебышёва-Эрмита и Чебышёва-Лагерра:

$$
\begin{aligned}
& Q_{n}(x)=\frac{(-1)^{n}}{(2 k)^{n}} e^{k x^{2}} \frac{d^{n} e^{-k x^{2}}}{d x^{n}}, \quad n=1,2, \ldots \\
& Q_{n}(x)=\frac{(-1)^{n}}{k^{n}} \frac{d^{n}\left(x^{n} e^{-k x}\right)}{d x^{n}},
\end{aligned}
$$

Отметим, что Эрмит рассмотрел многочлены, носящие его имя, только в 1864 году, а Лагерр - в 1879 году (см. [2]).

Тот факт, что исторически общие ортогональные многочлены впервые возникли в [1] непосредственно в связи с теорией непрерывных дробей отмечен и в монографии Сегё [6, гл. III, п. 3.5, c. 66].

2. Пусть $q_{n}(\lambda)=k_{n} \lambda^{n}+\cdots, k_{n}>0$, - соответствующие мере $\mu$ ортонормированные многочлены:

$$
\int q_{n}(x) q_{k}(x) d \mu(x)=\delta_{n k} .
$$

Для них справедливо равенство $q_{n}(\lambda)=Q_{n}(\lambda) /\left(a_{1} \ldots a_{n}\right)$ и выполняется следующее трехчленное рекуррентное соотношение:

$$
a_{n} q_{n}(\lambda)=\left(\lambda-b_{n}\right) q_{n-1}(\lambda)-a_{n-1} q_{n-2}(\lambda), \quad n=2,3, \ldots,
$$

где

$$
\begin{gathered}
q_{0}(\lambda) \equiv 1, \quad a_{1} q_{1}(\lambda)=\lambda-b_{1}, \\
a_{n}=\int \lambda q_{n-1}(\lambda) q_{n}(\lambda) d \mu(\lambda)=\frac{k_{n-1}}{k_{n}}, \\
b_{n}=\int \lambda q_{n-1}^{2}(\lambda) d \mu(\lambda) .
\end{gathered}
$$

Известно, что и наоборот, если некоторая последовательность многочленов $\left\{q_{n}(\lambda)\right\}_{n \in \mathbb{N}_{0}}, \mathbb{N}_{0}=\mathbb{N} \cup\{0\}$, удовлетворяет соотношениям (9)-(10) с вещественными ограниченными коэффициентами $b_{n}$ и $a_{n}, a_{n}>0$, то в соответствии с теоремой Фавара (см., например, [6]-[7]) эти многочлены ортонормированы относительно некоторой положительной борелевской меры $\mu$ на $\mathbb{R}$. Если же, кроме того, коэффициенты $a_{n}$ и $b_{n}$ имеют пределы, точнее

$$
a_{n} \rightarrow \frac{1}{2}, \quad b_{n} \rightarrow 0 \quad \text { при } n \rightarrow \infty,
$$


то в соответствии с теоремой Блюменталя (см., например, [7]-[8]) носитель меры $\mu$ состоит из отрезка $\Delta=[-1,1]$ и не более, чем счетного множества точек на $\mathbb{R} \backslash \Delta$, которые могут накапливаться лишь к концам этого отрезка.

Для дальнейшего использования соотношений (9)-(10) удобно переписать их в следующем виде:

$$
\begin{aligned}
a_{0} q_{-1}(\lambda)+b_{1} q_{0}(\lambda)+a_{1} q_{1}(\lambda) & =\lambda q_{0}(\lambda), \\
a_{n-1} q_{n-2}(\lambda)+b_{n} q_{n-1}(\lambda)+a_{n} q_{n}(\lambda) & =\lambda q_{n-1}(\lambda),
\end{aligned}
$$

где $n=2,3 \ldots, a_{0}=1, q_{-1}(\lambda) \equiv 0, q_{0}(\lambda) \equiv 1$. Для соответствующих ортонормированным многочленам $q_{n}(\lambda)$ многочленов второго рода $p_{n}(\lambda)$ и функций второго рода $r_{n}(\lambda)$ имеем:

$$
\begin{aligned}
p_{n}(\lambda) & :=\int \frac{q_{n}(\lambda)-q_{n}(x)}{\lambda-x} d \mu(x), \\
r_{n}(\lambda) & :=\int \frac{q_{n}(x) d \mu(x)}{\lambda-x}=\frac{1}{q_{n}(\lambda)} \int \frac{q_{n}^{2}(x) d \mu(x)}{\lambda-x} .
\end{aligned}
$$

Тем самым (см. (6)-(7))

$$
r_{n}(\lambda)=\left(q_{n} \widehat{\mu}-p_{n}\right)(\lambda)=\frac{1}{k_{n} \lambda^{n+1}}+\cdots, \quad \lambda \rightarrow \infty .
$$

Функции $p_{n}(\lambda)$ и $r_{n}(\lambda)$ также удовлетворяют рекуррентным соотношениям (13), но с другими начальными условиями:

$$
p_{-1}(\lambda) \equiv-1, \quad p_{0}(\lambda) \equiv 0, \quad r_{-1}(\lambda) \equiv 1, \quad r_{0}(\lambda)=\widehat{\mu}(\lambda) .
$$

Хорошо известно, что с теми же последовательностями $\left\{b_{n}\right\}$ и $\left\{a_{n}\right\}, a_{n}>0$, можно связать ограниченный самосопряженный оператор в пространстве $\ell^{2}=\ell^{2}(\mathbb{N})$. Точнее, пусть $\left\{\mathbf{e}_{j}\right\}_{j=1}^{\infty}$ - стандартный базис в $\ell^{2}=\ell^{2}(\mathbb{N})$. Определим оператор Якоби $J: \ell^{2} \rightarrow \ell^{2}$ на этом базисе следующим образом:

$$
\begin{aligned}
& J \mathbf{e}_{1}=b_{1} \mathbf{e}_{1}+a_{1} \mathbf{e}_{2}, \\
& J \mathbf{e}_{n}=a_{n-1} \mathbf{e}_{n-1}+b_{n} \mathbf{e}_{n}+a_{n} \mathbf{e}_{n+1}, \quad n=2,3, \ldots .
\end{aligned}
$$

Оператору $J$ соответствует бесконечная трехдиагональная матрица Якоби:

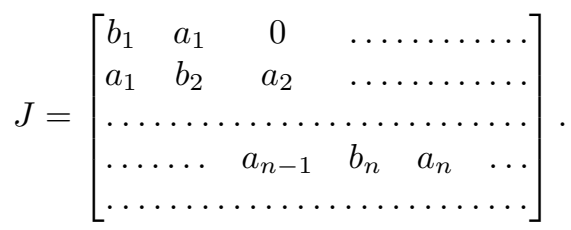


Пусть $E_{\lambda}-$ спектральное разложение оператора $J$. Тогда $\mu(\lambda):=$ $\left\langle E_{\lambda} \mathbf{e}_{1}, \mathbf{e}_{1}\right\rangle$ - неотрицательная неубывающая функция на $\mathbb{R}, d \mu(\lambda)$ - спектральная мера $J$. Функция $m(\lambda):=\left\langle(J-\lambda)^{-1} \mathbf{e}_{1}, \mathbf{e}_{1}\right\rangle$ называется функцией Вейля оператора $J$. Используя спектральную теорему, получаем для $m(\lambda)$ следующее представление:

$$
m(\lambda)=\int \frac{d \mu(x)}{x-\lambda}=-\widehat{\mu}(\lambda), \quad \lambda \in \widehat{\mathbb{C}} \backslash \sigma(J),
$$

где $\sigma(J)=\operatorname{supp} \mu-$ спектр $J$. Пусть теперь $q_{n}(\lambda), n=0,1, \ldots$, - последовательность многочленов, определенная рекуррентными соотношениями (13). Нетрудно увидеть, что $\mathbf{e}_{n}=q_{n-1}(J) \mathbf{e}_{1}$. Следовательно, в соответствии со спектральной теоремой, имеем:

$$
\begin{aligned}
\delta_{k j} & =\left\langle\mathbf{e}_{k}, \mathbf{e}_{j}\right\rangle=\left\langle q_{k-1}(J) \mathbf{e}_{1}, q_{j-1}(J) \mathbf{e}_{1}\right\rangle=\left\langle q_{j-1}(J) q_{k-1}(J) \mathbf{e}_{1}, \mathbf{e}_{1}\right\rangle \\
& =\int q_{k-1}(\lambda) q_{j-1}(\lambda) d \mu(\lambda) .
\end{aligned}
$$

Тем самым, $\left\{q_{n}\right\}_{n \in \mathbb{N}_{0}}-$ последовательность полиномов, ортонормированных относительно спектральной меры $d \mu(\lambda)$.

Предположим теперь, что выполнены условия теоремы Блюменталя, т.е.

$$
a_{n} \rightarrow \frac{1}{2}, \quad b_{n} \rightarrow 0 \quad \text { при } n \rightarrow \infty .
$$

Тогда $J=J_{0}+A$, где

$$
J_{0}=\left[\begin{array}{cccc}
0 & 1 / 2 & 0 & \ldots \\
1 / 2 & 0 & 1 / 2 & \ldots \\
\ldots & \ldots & \ldots & \ldots
\end{array}\right] .
$$

а $A$ - компактный оператор в $\ell^{2}$. Тем самым, $J$ - компактное возмущение оператора $J_{0}$. Для оператора $J_{0}$ спектр $\sigma\left(J_{0}\right)=[-1,1]$, а соответствующая спектральная мера $d \mu_{0}=\frac{2}{\pi} \sqrt{1-x^{2}} d x$. В таком случае, непосредственно из теоремы Вейля о компактном возмущении оператора вытекает, что спектр $J$ состоит из отрезка $\Delta$ и не более чем счетного множества точек на $\mathbb{R} \backslash \Delta$, которые могут накапливаться лишь к концам этого отрезка.

Вышеуказанная связь между теорией ортогональных многочленов и теорией операторов Якоби ${ }^{3}$ давно и хорошо известна,

${ }^{3} \mathrm{~B}$ [7], [12]-[13] такой оператор называется дискретным оператором Штурма-Лиувилля. 
см. [7]-[12]. Осознание этой связи в значительной степени стимулировало исследования и привело к новым интересным результатам как в той, так и в другой теории (см. прежде всего [7]-[16], где имеются дальнейшие ссылки). Однако как правило исследования шли в направлении "от дискретных операторов к ортогональным многочленам": с помощью общих методов теории операторов устанавливались определенные свойства оператора Якоби, порожденного рекуррентными соотношениями (16), а отсюда уже выводились те или иные асимптотические свойства ортогональных многочленов. В настоящей работе мы идем "от ортогональных многочленов к дискретным операторам" и в таком подходе следуем Е.М.Никишину [7], который предложил для исследования асимптотических свойств решений разностного аналога уравнения Штурма-Лиувилля использовать методы, развитые в теории ортогональных многочленов, теории непрерывных дробей и аппроксимаций Паде. В [7] он ограничился только классическим случаем (12). В дальнейшем такой подход успешно применялся им и его учениками [12]-[14] и в более общей ситуации.

3. Рассмотрим следующую систему трехчленных рекуррентных соотношений - разностный аналог уравнения ШтурмаЛиувилля на полуоси (см., например, [7], [11]):

$$
\begin{aligned}
& a_{0} y_{0}+b_{1} y_{1}+a_{1} y_{2}=\lambda y_{1}, \\
& a_{1} y_{1}+b_{2} y_{2}+a_{2} y_{3}=\lambda y_{2} \text {, } \\
& \text {........................... } \\
& a_{n-1} y_{n-1}+b_{n} y_{n}+a_{n} y_{n+1}=\lambda y_{n}, \\
& \text {............................. }
\end{aligned}
$$

где $a_{0}=1$, все $a_{n}, b_{n}-$ вещественны, $a_{n}>0$ и $\sup _{n} a_{n}<\infty$, $\sup \left|b_{n}\right|<\infty$ (выбор $a_{0}=1$ соответствует нормировке меры $\mu$ как

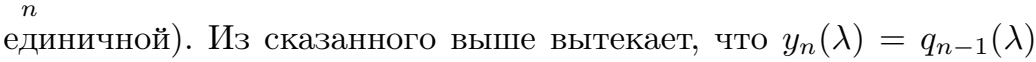
- решение системы (17) с начальными условиями $y_{0}(\lambda) \equiv 0$, $y_{1}(\lambda) \equiv 1, y_{n}(\lambda)=p_{n-1}(\lambda)$ - решение $(17)$ с начальными условиями $y_{0}(\lambda) \equiv-1, y_{1}(\lambda) \equiv 0$, наконец, $y_{n}(\lambda)=r_{n-1}(\lambda)-$ решение с начальными условиями $y_{0}(\lambda) \equiv 1, y_{1}(\lambda)=\widehat{\mu}(\lambda)$.

Положим $\lambda=\frac{1}{2}(z+1 / z)$, где $z \in \mathbb{D}, \mathbb{D}:|z|<1$. Семейство $\left\{u_{n}(z)\right\}_{n \in \mathbb{N}_{0}}, z \in \mathbb{D}$, называется решением Йоста для (17) (см. прежде всего [9]), если при каждом $z \in \mathbb{D}$ таком, что $\lambda=\frac{1}{2}(z+1 / z) \notin \sigma(J)$, последовательность $\left\{u_{n}(z)\right\}_{n \in \mathbb{N}_{0}}$ является 
решением системы (17) и имеет место следующее асимптотическое соотношение:

$$
z^{-n} u_{n}(z) \rightarrow 1 \quad \text { при } n \rightarrow \infty, z \in \mathbb{D} \text {; }
$$

функция $u_{0}(z), z \in \mathbb{D}$, называется функиией Йоста. В [9] было установлено, что если решение Йоста существует, то асимптотика ортогональных многочленов $q_{n}(\lambda)$ выражается явно в терминах функции Йоста $u_{0}(z)$ ([9], формулы (IV.8), (IV.17')). Позднее в [10] было доказано, что решение Йоста существует при условии суммируемости первого момента

$$
\sum_{n=1}^{\infty} n\left(\left|a_{n}-\frac{1}{2}\right|+\left|b_{n}\right|\right)<\infty .
$$

Если условие (18) не выполняется, то решение Йоста может и не существовать. В [15] по аналогии с непрерывным случаем вместо решения Йоста было предложено рассматривать решение Вейля $\left\{w_{n}(z)\right\}_{n \in \mathbb{N}_{0}}$ системы $(17)$ :

$$
w_{n}(z)=\left\langle\mathbf{e}_{n},(\lambda-J)^{-1} \mathbf{e}_{1}\right\rangle, \quad n=1,2, \ldots,
$$

где

$$
\lambda=\frac{1}{2}\left(z+\frac{1}{z}\right) \notin \sigma(J), \quad z \in \mathbb{D},
$$

a $w_{0}(z) \equiv 1$. Если решение Йоста существует, то между ним и решением Вейля имеется следующая легко устанавливаемая связь. Обозначим $v_{n}(z):=z^{-n} w_{n}(z)$. Тогда существует

$$
v_{\infty}(z)=\lim _{n \rightarrow \infty} z^{-n} w_{n}(z) \neq 0 \text { при } z \in \mathbb{D} \backslash \mathbb{R}
$$

и $v_{\infty}(z)=1 / u_{0}(z)$, где $u_{0}(z)$ - функция Йоста. Ввиду такой связи асимптотику типа (20) в [15] было предложено называть acusnmотикой Йоста. Этой терминологии мы будем придерживаться и в настоящей работе.

Так как $w_{0}(z) \equiv 1, w_{1}(z)=\widehat{\mu}(\lambda)$ и $\left\{w_{n}(z)\right\}$ - решение системы $(17)$, то $w_{n}(z)=r_{n-1}(\lambda)$ при всех $n=1,2, \ldots$ Таким образом, имеем: $w_{n}(z)=\left(q_{n-1} \widehat{\mu}-p_{n-1}\right)(\lambda)=c_{n}(\lambda)-m(\lambda) s_{n}(\lambda)$, где $c_{n}$ и $s_{n}$ - решения (17) соответственно типа косинуса и синуса, а $m(\lambda)$ - функция Вейля. Отметим, что используемое здесь определение 
решения Вейля несколько отличается от стандартного (см., например, [17], где имеются дальнейшие ссылки).

Под сильной (или типа Сегё) асимптотикой для ортогональных многочленов $q_{n}(\lambda)$ при $\lambda \notin \sigma(J)$ будем понимать следующее. При $n \rightarrow \infty$ существует отличный от нуля предел произведения $c_{n}(z):=z^{n} q_{n}(\lambda)$ :

$$
\lim _{n \rightarrow \infty} c_{n}(z)=c_{\infty}(z) \neq 0, \quad z \in \mathbb{D}, \quad \lambda=\frac{1}{2}\left(z+\frac{1}{z}\right) \notin \sigma(J) .
$$

Один из полученных в [15] в этом направлении результатов состоит в том, что при самых общих предположениях (12) на коэффициенты $a_{n}$ и $b_{n}$ оператора $J$ существование сильной асимптотики для ортогональных многочленов оказывается эквивалентным существованию асимптотики Йоста для решения Вейля. Точнее, справедлива следующая

Teоpema. (см. [15], теорема 2.2) Пусть $J$ - матрица Якоби, для коэффициентов $a_{n}$ u $b_{n}$ которой имеем: $a_{n} \rightarrow 1 / 2, b_{n} \rightarrow 0$ при $n \rightarrow \infty$. Если $z \in \mathbb{D}$ таково, что $\lambda=\frac{1}{2}(z+1 / z)$ не является собственным значением $J$, то существование предела $v_{\infty}(z) \neq 0$ для последовательности $v_{n}(z)$ эквивалентно существованию предела $c_{\infty}(z) \neq 0$ для последовательности $c_{n}(z)$. При этом величины $v_{\infty}(z)$ и $c_{\infty}(z)$ связаны соотношением:

$$
c_{\infty}(z) v_{\infty}(z)=\frac{1}{1-z^{2}} .
$$

В силу сказанного непосредственно из (21) вытекает следующая формула сравнительной асимптотики для $\left\{q_{n}(\lambda)\right\}_{n \in \mathbb{N}}$ и $\left\{r_{n}(\lambda)\right\}_{n \in \mathbb{N}}$ :

$$
q_{n}(\lambda) r_{n}(\lambda) \rightarrow \frac{z}{1-z^{2}}=\frac{1}{\sqrt{\lambda^{2}-1}}, \quad z \in \mathbb{D}, \quad \lambda \notin \sigma(J) .
$$

Таким образом, при самых общих предположениях (12) относительно оператора $J$ асимптотические свойства двух фундаментальных решений системы (17) оказываются тесно связанными. Отметим, что правая часть $(22)$ не зависит от $J$, а тем самым и от спектральной меры $\mu$.

Подчеркнем однако, что все приведенные выше результаты относятся к случаю, когда выполняется условие (12), т.е. параметры 
$a_{n}$ и $b_{n}$ uмеют пределы. При этом условии для носителя спектральной меры (т.е. меры ортогональности) $\mu$ имеем: ess supp $\mu=$ $[-1,1]$. Таким образом, условием (12) круг рассматриваемых вопросов заранее сужается ограничением ess supp $\mu=[-1,1]$, накладываемым явно или неявно на класс изучаемых на полуоси операторов Якоби. Ситуация, когда в ess supp $\mu$ имеется хотя бы одна лакуна, имеет принципиальные отличия от классического случая "возмущения на постоянном фоне", задаваемого условием (12), и требует иных методов исследования. Подчеркнем, что речь идет о достаточно общей ситуации, вообще говоря, отличной от случая "возмущения на периодическом фоне".

В настоящей работе рассматривается класс операторов Якоби, порождаемых борелевской мерой $\mu$ с носителем на $\mathbb{R}$, состоящим из конечного числа отрезков. При этом предполагается, что мера $\mu$ абсолютно непрерывна относительно меры Лебега и имеет специальный вид (см. ниже $\S 2$, п. 1), на сами же отрезки не накладывается никаких условий, в частности, они могут находиться "в общем положении". Цель настоящей работь - при достаточно общих условиях на меру $\mu$ получить формулу сравнительной acuмnтотики двух фундаментальных решений соответствующего этой мере разностного уравнения (17): многочленов, ортогональных относительно $\mu$, и решения Вейля, а также - acusnтотические формулы следов для коэффициентов $a_{n}$ и $b_{n}$ такого уравнения. Подчеркнем еще раз, что в своем подходе мы следуем Е.М.Никишину [7], который предложил для исследования асимптотических свойств решений дискретного аналога оператора Штурма-Лиувилля использовать методы, развитые в теории ортогональных многочленов и теории аппроксимаций Паде. Отметим, что речь идет об операторе Якоби, рассматриваемом на полуоси, т.е. в пространстве $\ell^{2}(\mathbb{N})$. Пространство $\ell^{2}(\mathbb{Z})$ мы здесь не рассматриваем, поэтому и не обсуждаем полученных для этого случая (в том числе и в [7]) результатов.

Тот факт, что асимптотические свойства двух независимых решений разностного уравнения (17) оказываются тесно связанными и в более общей ситуации, чем задано условиями (12), давно и хорошо известен. Наиболее очевидный пример этому дает классическая теорема Маркова (см. [2], [6, гл. III, п. 3.5], [18]) о сходимости чебышевских непрерывных дробей для функций вида (1), в которой фактически утверждается следующее. Пусть $\left\{y_{n}^{(1)}(\lambda)\right\}$ - решение системы (17) с начальными данными $(0,1)$, a $\left\{y_{n}^{(2)}(\lambda)\right\}$ 
- решение этой системы с начальными данными $(-1,0)$. Тогда (ср. [16], формула (4.2))

$$
\frac{y^{(2)}(\lambda)}{y^{(1)}(\lambda)} \rightarrow \widehat{\mu}(\lambda) \quad \text { равномерно внутри } \widehat{\mathbb{C}} \backslash \Delta
$$

$\Delta$ - выпуклая оболочка $\operatorname{supp} \mu$. Другой пример - результат A. A. Гончара [20] также о сходимости чебышевских непрерывных дробей, но - с комплексными коэффициентами $a_{n} \neq 0$ и $b_{n}$. Этот результат допускает следующую интерпретацию в рамках теории разностного оператора (17) с комплексными коэффициентами $a_{n} \neq 0$ и $b_{n}$. Обозначим как и выше $\left\{y_{n}^{(1)}(\lambda)\right\}-$ решение системы (17) с начальными данными $(0,1)$, a $\left\{y_{n}^{(2)}(\lambda)\right\}-$ решение этой системы с начальными данными $(-1,0)$. Пусть $K_{1} \Subset \mathbb{C}-$ множество всех предельных точек нулей функций $y_{n}^{(1)}(\lambda), \widehat{K_{1}}-$ его выпуклая оболочка. Тогда множество $K_{2}$ предельных точек нулей функций $y_{n}^{(2)}(\lambda)$ устроено следующим образом: вне компакта $\widehat{K_{1}}$ оно может иметь лишь изолированные точки. При этом

$$
\frac{y^{(2)}(\lambda)}{y^{(1)}(\lambda)} \rightarrow \widehat{\mu}(\lambda) \quad \text { равномерно внутри } \widehat{\mathbb{C}} \backslash \widehat{K_{1}}
$$

Теорема Маркова является очевидным следствием этого результата Гончара.

Отметим, что $r_{n-1}(\lambda)$ является "минимальным" решением (17) в следующем смысле. Если $y_{n}(\lambda)$ - любое другое решение $(17)$, линейно независимое с $r_{n-1}(\lambda)$, то $r_{n-1}(\lambda) / y_{n}(\lambda) \rightarrow 0$ при $n \rightarrow \infty$ и $\lambda \notin \sigma(J)$.

4. Прежде чем формулировать основные результаты настоящей работы, сделаем некоторые предварительные пояснения.

Пусть $\left\{w_{n}(z)\right\}_{n \in \mathbb{N}}$ - последовательность функций, определенных соотношениями (19). Используя равенства $\mathbf{e}_{n+1}=q_{n}(J) \mathbf{e}_{1}$, (14) и спектральную теорему получаем следующее представление для $w_{n+1}(z)$ при $\lambda=\frac{1}{2}(z+1 / z), \lambda \notin \sigma(J)=\operatorname{supp} \mu$ и $n=0,1, \ldots$ :

$$
\begin{aligned}
w_{n+1}(z) & =\left\langle\mathbf{e}_{n+1},(\lambda-J)^{-1} \mathbf{e}_{1}\right\rangle=\left\langle q_{n}(J) \mathbf{e}_{1},(\lambda-J)^{-1} \mathbf{e}_{1}\right\rangle \\
& =\left\langle q_{n}(J)(\lambda-J)^{-1} \mathbf{e}_{1}, \mathbf{e}_{1}\right\rangle=\int \frac{q_{n}(x)}{\lambda-x} d \mu(x)=r_{n}(\lambda) .
\end{aligned}
$$


Таким образом, последовательность функций $w_{n}(z)$, определенная (19) и начальным условием $w_{0}(z) \equiv 1$, совпадает с последовательностью функций второго рода $r_{n-1}(\lambda)$, если положить $r_{-1}(\lambda) \equiv 1$. Тем самым, доказано, что последовательность $\left\{w_{n}(z)\right\}_{n \in \mathbb{N}_{0}}$ - решение системы (17). Отметим, что для вронскиана двух решений $(17) r_{n}(\lambda)$ и $q_{n}(\lambda)$ имеем: $W_{n}(q, r)=a_{n}\left(q_{n} r_{n-1}-\right.$ $\left.r_{n} q_{n-1}\right)=1$.

Как было отмечено выше, при условиях (12) справедлива формула сравнительной асимптотики для двух независимых решений $\left\{q_{n-1}(\lambda)\right\}_{n \in \mathbb{N}_{0}}$ и $\left\{r_{n-1}(\lambda)\right\}_{n \in \mathbb{N}_{0}}$ разностного уравнения (17):

$$
q_{n}(\lambda) r_{n}(\lambda) \rightarrow \frac{1}{\sqrt{\lambda^{2}-1}} \quad \text { при } n \rightarrow \infty, \text { где } \lambda \in \widehat{\mathbb{C}} \backslash \sigma(J) .
$$

В [21] Е. А. Рахмановым было, в частности, установлено, что соотношение (23) имеет место для мер $\mu$ таких, что $\operatorname{supp} \mu=[-1,1]$ и $\mu^{\prime}>0$ почти всюду на $[-1,1]$. А. А. Гончар в [18] доказал, что $(23)$ остается справедливым, если к такой мере добавить конечное число точечных масс, расположенных вне отрезка $[-1,1]$. Аналог теоремы Гончара оказывается справедлив [19] и для случая, когда в $\operatorname{ess} \operatorname{supp} \mu$ имеется конечное число лакун $\left(\alpha_{j}, \beta_{j}\right), j=1,2, \ldots, g$, т.е. носитель меры $\mu$ состоит из $\operatorname{ess} \operatorname{supp} \mu=[-1,1] \backslash \bigcup_{j=1}^{g}\left(\alpha_{j}, \beta_{j}\right)$ и конечного числа точек на $\mathbb{R}$, расположенных вне отрезка $[-1,1]$. Основная цель настоящей работы - получить аналог формулы (23) для случая, когда носитель меры $\mu$ cocmoum из нескольких отрезков.

Настоящая работа является фактически продолжением работ [22] и [23], §7, результаты которых относились прежде всего к теории сходимости аппроксимаций Паде и были основаны на формулах сильной асимптотики для ортогональных многочленов $Q_{n}(\lambda)$ и функций второго рода $R_{n}(\lambda)$, рассматриваемых и изучаемых в этих работах соответственно как знаменатели диагональных аппроксимаций Паде $P_{n} / Q_{n}$ марковской функции $\widehat{\mu}$ и функции остатка (см. (6)). Как хорошо известно, в $g$ лакунах $\left(\alpha_{j}, \beta_{j}\right)$ между отрезками носителя меры $\mu$ могут располагаться $\leqslant g$ нулей ортогональных многочленов $Q_{n}$ (иначе говоря, “ложных" полюсов диагональных аппроксимаций Паде, подробнее об этом понятии см. [24], где имеются дальнейшие ссылки), которые в "общем положении" всюду плотны в этих лакунах (см. [22], [23]). В [22] было показано, что этот же результат имеет место и для 
нулей $R_{n}$, т.е. для дополнительных точек интерполяции марковской функции $\widehat{\mu}$ диагональными аппроксимациями Паде $P_{n} / Q_{n}$. Точнее, в [22] было установлено, что по заданному набору отрезков, составляющих ess supp $\mu$, некоторым естественным образом определяется нелинейная система из $g$ дифференциальных уравнений относительно непрерывного переменного $t$, аналогичная известной системе Дубровина. Эта система интегрируется преобразованием Абеля, ее решение задается квазипериодической функцией от непрерывного переменного $t$ со значениями в $g$-мерном вещественном торе. Оказывается, что динамика этого решения на торе после подстановки вместо непрерывного переменного $t \in \mathbb{R}$ дискретного переменного $n \in \mathbb{N}$ полностью определяет как динамику нулей $Q_{n}$, так и нулей $R_{n}$, оказавшихся в лакунах между отрезками. При этом "начальные данные" для решения однозначно определяются мерой $\mu$, а роль периодов $\omega_{j}$ в описании квазипериодичности решения выполняют взятые в бесконечно удаленной точке гармонические меры $\omega_{j}(\infty), j=1,2, \ldots, g+1$, отрезков, составляющих ess supp $\mu$. Отсюда уже, поскольку в общем положении гармонические меры $\omega_{j}(\infty)$ рационально независимы, и вытекали упомянутые выше утверждения о том, что как нули $Q_{n}$, так и нули $R_{n}$ всюду плотны в лакунах между отрезками. Однако как [22], так и [23] были посвящены в первую очередь изучению сходимости диагональных аппроксимаций Паде, которое фактически сводится к исследованию асимптотического поведения их полюсов, т.е. нулей ортогональных полиномов $Q_{n}$. Для вывода в интересующем нас случае нескольких отрезков аналога формулы (23) сравнительной асимптотики ортогональных многочленов и функций второго рода нам придется проводить полное исследование асимптотики их нулей, причем - одновременно, т.е. при одних и тех же значениях индекса $n \in \mathbb{N}$ (ср. [22], теоремы 1 и 3). Вывод соответствующих асимптотических формул проводится по следующей ставшей уже стандартной схеме (см. [22]-[27]). Сначала при произвольном $n \in \mathbb{N}$ в "явном виде" строится решение специальной краевой задачи Римана на двулистной гиперэллиптической римановой поверхности; это решение мы будем в дальнейшем называть $\Psi$-функцией. ${ }^{4}$ Затем выводится (обобщенное) сингулярное интегральное уравнение Наттолла (см. [28], где это уравнение получено впервые для классического случая одного отрезка), связывающее при каждом $n \in \mathbb{N}$ между собой $\Psi$-функцию,

\footnotetext{
${ }^{4}$ Разумеется эта функция зависит от $n$; подробнее см. ниже.
} 
ортогональные многочлены $Q_{n}$ и функцию остатка $R_{n}$ (см. (35)$(36))$. Из определения и явного представления $\Psi$-функции вытекает, что она обладает специальными свойствами. На этом и основываются дальнейшие рассуждения.

Настоящая работа устроена следующим образом. Во втором параграфе формулируются основные результаты работы. Третий параграф посвящен доказательству теоремы 1 , четвертый - доказательству теоремы 2. В приложении А приводятся некоторые стандартные сведения о гиперэллиптических римановых поверхностях, используемые в работе. В приложении В выводится явное представление $\Psi$-функции в терминах абелевых дифференциалов. В приложении $\mathrm{C}$ кратко излагается содержание работы Чебышёва [1], в которой он впервые ввел общие ортогональные многочлены.

Автор выражает благодарность И.Е. Егоровой за полезные обсуждения.

\section{§ 2. Формулировка основных результатов}

1. Теперь нам будет удобно ввести некоторые новые обозначения. Будем считать, что носитель меры $\mu$ состоит из непересекающихся отрезков $\Delta_{j}=\left[e_{2 j-1}, e_{2 j}\right], j=1, \ldots, g+1$, расположенных на вещественной прямой $\mathbb{R}, g \geqslant 1, e_{1}<\cdots<e_{2 g+2}$. Меру $\mu$ будем считать абсолютно непрерывной относительно меры Лебега на $S:=\bigsqcup_{j=1}^{g+1} \Delta_{j}, S=\operatorname{supp} \mu$, и такой, что

$$
\frac{d \mu}{d x}=\frac{1}{\pi} \frac{\rho(x)}{\sqrt{-h(x)}}>0 \quad \text { на } S,
$$

где $h(\lambda)=\prod_{j=1}^{2 g+2}\left(\lambda-e_{j}\right)$, причем в области $D=\widehat{\mathbb{C}} \backslash S$ выбрана та ветвь квадратного корня, которая положительна при положительных значениях аргумента. Тем самым, $\sqrt{-h(x)}>0$ при $x \in\left[e_{2 g+1}, e_{2 g+2}\right]$, на остальных отрезках значение корня определяется аналитическим продолжением. Весовая функция $\rho$ предполагается голоморфной и отличной от нуля на $S$. Тем самым,

$$
\widehat{\mu}(\lambda)=\frac{1}{\pi} \int_{S} \frac{1}{\lambda-x} \frac{\rho(x) d x}{\sqrt{-h(x)}}, \quad \lambda \notin \operatorname{supp} \mu .
$$

Через $\omega_{k}(\lambda)=\omega\left(\lambda ; \Delta_{k}, D\right), k=1, \ldots, g+1$, будем обозначать гармоническую меру (в точке $\lambda \in D$ ) отрезка $\Delta_{k}$ относительно 
области $D, g(\lambda, \infty)=g_{D}(\lambda, \infty)-$ функция Грина области $D=$ $\widehat{\mathbb{C}} \backslash S$ с особенностью в бесконечно удаленной точке $\lambda=\infty$.

Пусть $\mathfrak{R}$ - гиперэллиптическая риманова поверхность рода $g$, заданная уравнением $w^{2}=h(\lambda)$. Будем считать, что $\mathfrak{R}$ реализована как двулистное разветвленное в точках $\left\{e_{j}\right\}$ накрытие римановой сферы $\widehat{\mathbb{C}}$ таким образом, что переход с одного листа на другой осуществляется по верхнему $\Delta_{j}^{+}$и нижнему $\Delta_{j}^{-}$берегам отрезков $\Delta_{j}$. Тем самым, над каждой точкой $\widehat{\mathbb{C}}$ за исключением точек ветвления $\left\{e_{j}\right\}$ лежат ровно две точки римановой поверхности, а каждому отрезку $\Delta_{j}$ соответствует на $\mathfrak{R}$ замкнутая аналитическая (в комплексной структуре $\mathfrak{R})$ кривая $\boldsymbol{\Gamma}_{j}, j=1, \ldots, g+1,-$ цикл на $\mathfrak{R}$; положим $\boldsymbol{\Gamma}=\bigcup_{j=1}^{g+1} \boldsymbol{\Gamma}_{j}$. Выбранная в $D=\widehat{\mathbb{C}} \backslash S$ ветвь квадратного корня удовлетворяет условию $\sqrt{h(\lambda)} / \lambda^{g+1} \rightarrow 1$ при $\lambda \rightarrow \infty$. Функция $w: w^{2}=h(\lambda)$ однозначна на $\mathfrak{R}$. Первым (открытым) листом $D^{(1)}$ поверхности $\mathfrak{R}$ будем считать тот, на котором $w=\sqrt{h(\lambda)}$. На втором листе $D^{(2)}$ имеем: $w=-\sqrt{h(\lambda)}$. Для точек римановой поверхности $\mathfrak{R}$ будем использовать обозначение $\boldsymbol{\lambda}=(\lambda, w)$, где $w= \pm \sqrt{h(\lambda)} ;$ при этом для точек первого листа $\lambda^{(1)}=(\lambda, \sqrt{h(\lambda)})$, а для точек второго $\lambda^{(2)}=(\lambda,-\sqrt{h(\lambda)})$. Вместо $\boldsymbol{\lambda}=(\lambda, \pm \sqrt{h(\lambda)})$ иногда будем писать коротко $\boldsymbol{\lambda}=(\lambda, \pm)$. Область $D^{(1)}$ будем как правило отождествлять с "физической" областью $D$. Для $\boldsymbol{\lambda}=\lambda^{(1)}$ будем часто писать просто $w(\lambda)$ вместо $w(\boldsymbol{\lambda})$; тем самым, приобретает смысл и запись $w^{ \pm}(x)=\sqrt{h(x \pm i 0)}, x \in S$. Каноническая проекция pr $: \mathfrak{R} \rightarrow \widehat{\mathbb{C}}$ определяется соотношением pr $\boldsymbol{\lambda}=\lambda$, в частности $\operatorname{pr} D^{(1)}=\operatorname{pr} D^{(2)}=D, \operatorname{pr} \boldsymbol{\Gamma}=S$. Замкнутые циклы на $\mathfrak{R}$, соответствующие замкнутым лакунам $\left[e_{2 j}, e_{2 j+1}\right], j=1, \ldots, g$, будем обозначать через $\mathbf{L}_{j}$. Тем самым, $\operatorname{pr} \mathbf{L}_{j}=\left[e_{2 j}, e_{2 j+1}\right]$.

Другие стандартные сведения о гиперэллиптических римановых поверхностях, которые нам здесь понадобятся, приведены в приложении А.

2. Рассмотрим следующую систему из $g$ дифференциальных уравнений относительно (неупорядоченного) набора $g$ точек $\boldsymbol{\lambda}_{1}(t), \ldots, \boldsymbol{\lambda}_{g}(t)$ на $\mathfrak{R}, t \in \mathbb{R}$ (или, эквивалентно, относительно дивизора $d(t)=\boldsymbol{\lambda}_{1}(t)+\cdots+\boldsymbol{\lambda}_{g}(t)$ на $\left.S^{g} \mathfrak{R}\right)$ :

$$
\begin{aligned}
\frac{d \lambda_{j}}{d t}= & -\frac{w\left(\boldsymbol{\lambda}_{j}\right)}{\prod_{k \neq j}\left(\lambda_{j}-\lambda_{k}\right)} \\
& \times \int_{e_{2 g+2}}^{+\infty} \frac{\prod_{k \neq j}\left(x-\lambda_{k}\right)}{w(x)} d x, \quad j=1,2, \ldots, g .
\end{aligned}
$$


Как показано в [22], теорема 2 (см. также п. 6 приложения В) если все $\lambda_{j}(0)=\operatorname{pr} \boldsymbol{\lambda}_{j}(0) \in\left[e_{2 j}, e_{2 j+1}\right], j=1, \ldots, g$, то система (26) интегрируется в явном виде преобразованием Абеля, ее решение задается квазипериодической функцией непрерывного переменного $t$ с группой периодов $\omega_{1}(\infty), \ldots, \omega_{g}(\infty)$ и со значениями в $g$-мерном вещественном торе. Тем самым, все $\boldsymbol{\lambda}_{j}(t) \in \mathbf{L}_{j}$, $j=1, \ldots, g$, при $t>0$. Следовательно, все $\lambda_{j}(t)=\operatorname{pr} \boldsymbol{\lambda}_{j}(t) \in$ $\left[e_{2 j}, e_{2 j+1}\right], j=1, \ldots, g$, при $t>0$. Отметим, что система (26) приводит к системе уравнений Видома-Рахманова [29], [30]

$$
\begin{gathered}
\sum_{j=1}^{g} \varepsilon_{j} \omega_{k}\left(\lambda_{j}\right)=(g-2 t) \omega_{k}(\infty)-\frac{2}{\pi} \int_{S} \log |\rho(\zeta)| \frac{\partial \omega_{k}(\zeta)}{\partial n_{\zeta}^{+}} d \zeta \quad(\bmod 2), \\
k=1, \ldots, g
\end{gathered}
$$

описывающих движение дивизора $d(t)=\boldsymbol{\lambda}_{1}(t)+\cdots+\boldsymbol{\lambda}_{g}(t)$ на торе $\mathbf{L}_{1} \times \cdots \times \mathbf{L}_{g}$, где $\varepsilon_{j}= \pm 1$ в зависимости от $\boldsymbol{\lambda}_{j}=\left(\lambda_{j}, \pm\right)$. Учитывая геометрический смысл гармонической меры (см. ниже п. 7 приложения В), эти последние уравнения естественно трактовать следующим образом: дивизор $d(t)=\boldsymbol{\lambda}_{1}(t)+\cdots+\boldsymbol{\lambda}_{g}(t)$, удовлетворяющий системе $(26)$, движется с постоянной "угловой" скоростью (подробнее см. п. 6 приложения В).

Основным результатом настоящей работы является следующая

Теорема 1. При условии (24) для $\lambda \notin \operatorname{supp} \mu$ имеем:

$$
q_{n}(\lambda) r_{n}(\lambda)=\frac{\prod_{j=1}^{g}\left(\lambda-\lambda_{j}(n)\right)}{\sqrt{h(\lambda)}}+o\left(\delta^{n}\right) \text { nрu } n \rightarrow \infty,
$$

где $\delta \in(0,1)$ зависит от $\rho$, величины $\lambda_{1}(n), \ldots, \lambda_{g}(n)$ coответствуют решению $\boldsymbol{\lambda}_{1}(t), \ldots, \boldsymbol{\lambda}_{g}(t)$ системы $(26)$, взятому при $t=n \in \mathbb{N} ;$ начальные условия $\boldsymbol{\lambda}_{1}(0), \ldots, \boldsymbol{\lambda}_{g}(0)$ определяются мерой $\mu$, при этом, $\lambda_{j}(0) \in\left[e_{2 j}, e_{2 j+1}\right]$ для всех $j=1, \ldots, g$.

Доказательство теоремы 1 основано на стандартной технике [22], [23] исследования асимптотических свойств многочленов, ортогональных на нескольких отрезках, и состоит в сведении задачи об асимптотике к исследованию свойств $\Psi$-функции и свойств решения специального сингулярного интегрального уравнения Наттолла. Эти же свойства лежат в основе доказательства следующего результата. 
Теорема 2. При условии (24) справедливы следующие асимптотические формуль следов для коэффициентов $b_{n} u a_{n}$ разностного уравнения (17):

$$
\begin{aligned}
b_{n}= & \frac{1}{2} \sum_{j=1}^{2 g+2} e_{j}-\sum_{j=1}^{g} \lambda_{j}(n-1)+o\left(\delta^{n}\right), \quad n \rightarrow \infty, \\
a_{n}= & \operatorname{cap} S \cdot \exp \left\{\frac{1}{2} \sum_{j=1}^{g} g\left(\boldsymbol{\lambda}_{j}(n), \infty\right)\right. \\
& \left.-\frac{1}{2} \sum_{j=1}^{g} g\left(\boldsymbol{\lambda}_{j}(n-1), \infty\right)\right\}\left(1+o\left(\delta^{n}\right)\right), n \rightarrow \infty,
\end{aligned}
$$

где $\delta \in(0,1)$ зависит от $\rho$.

Здесь $g(\boldsymbol{\lambda}, \infty)$ - однозначное продолжение функции Грина $g_{D}(\lambda, \infty)$ области $D$ на всю риманову поверхность $\mathfrak{R}$.

Отметим, что асимптотическая формула (29) вытекает непосредственно из результатов Видома [30], §6, теорема 6.2 и $\S 9$, теорема 9.1, но с $o(1)$ вместо $o\left(\delta^{n}\right)$ как в $(29)$.

Теорема 1 может быть эквивалентным образом переформулирована в терминах функции Грина $G(\lambda, n, m):=\left\langle\mathbf{e}_{n},(\lambda-J)^{-1} \mathbf{e}_{m}\right\rangle$ оператора $J$. Точнее, пусть $G(\lambda, n, n)$ - диагональная функция Грина оператора $J$. Из спектральной теоремы вытекает, что $G(\lambda, n+1, n+1)=q_{n}(\lambda) r_{n}(\lambda)$. Следовательно, соотношение $(27)$ принимает вид:

$$
G(\lambda, n+1, n+1)=\frac{\prod_{j=1}^{g}\left(\lambda-\lambda_{j}(n)\right)}{\sqrt{h(\lambda)}}+o\left(\delta^{n}\right) \text { при } n \rightarrow \infty .
$$

3. Зафиксируем произвольное $n \in \mathbb{N}$ и рассмотрим на римановой поверхности $\mathfrak{R}$ следующую краевую задачу Римана.

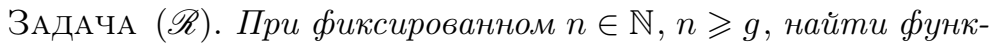
ииюю $\Psi=\Psi_{n}$ такую, что:

$1^{\circ} \Psi$ (кусочно) мероморфна на $\mathfrak{R} \backslash \boldsymbol{\Gamma}=D^{(1)} \sqcup D^{(2)}$;

$2^{\circ}$ дивизор $(\Psi)=(n-g) \infty^{(2)}+\boldsymbol{\lambda}_{1}+\cdots+\boldsymbol{\lambda}_{g}-n \infty^{(1)}$;

$3^{\circ}$ на $\boldsymbol{\Gamma}$ выполнено краевое условие: $\rho(x) \Psi^{+}(\mathbf{x})=\Psi^{-}(\mathbf{x}), \mathbf{x} \in \mathbf{\Gamma}$.

В п. $2^{\circ}$ точки $\boldsymbol{\lambda}_{j}$ - "свободные" нули $\Psi$-функции - зависят от $n$, под $\Psi^{+}(\mathbf{x})$ в п. $3^{\circ}$ понимаются предельные значения функции 
$\Psi(\boldsymbol{\lambda})$ при $D^{(1)} \ni \boldsymbol{\lambda} \rightarrow \mathbf{x} \in \boldsymbol{\Gamma}$, аналогичный смысл придается и $\Psi^{-}(\mathbf{x})$. Так как вес $\rho$ - марковский (см. (24)), то $\boldsymbol{\lambda}_{j} \in \mathbf{L}_{j}$, т.е. в каждой лакуне $L_{j}=\left[e_{2 j}, e_{2 j+1}\right]$ лежит ровно по одной точке $\lambda_{j}$. При этом допускается, чтобы какая-нибудь из точек $\boldsymbol{\lambda}_{j} \in \boldsymbol{\Gamma}$, т.е. совпадала бы с концом лакуны: $\boldsymbol{\lambda}_{j}=e_{2 j}$ или $\boldsymbol{\lambda}_{j}=e_{2 j+1}$. В таком случае эта точка считается как нулем $\Psi^{+}$, так и нулем $\Psi^{-}$. Как будет видно ниже, благодаря тому, что функция $\rho$ голоморфна на $\boldsymbol{\Gamma}$, в такой ситуации сохраняются все основные свойства $\Psi$ функции, в том числе - представление (30)-(33) (см. ниже).

Функция $\Psi$, решающая задачу $(\mathscr{R})$, всегда существует, при этом так как род $g$ поверхности $\mathfrak{R}$ положителен, то нули и полюсы $\Psi$ на $\mathfrak{R}$ оказываются связанными определенными соотношениями, аналогичными соотношениям Абеля для мероморфной функции на $\mathfrak{R}$, а дивизор $d=\boldsymbol{\lambda}_{1}+\cdots+\boldsymbol{\lambda}_{g}$ является решением проблемы обращения Якоби. Анализ данных этой проблемы показывает, что при оговоренном выше условии (24) на вес $\rho$ ее решение всегда таково, что $\lambda_{j}=\operatorname{pr} \boldsymbol{\lambda}_{j} \in\left[e_{2 j}, e_{2 j+1}\right]$, т.е. в каждой замкнутой лакуне $L_{j}$ между отрезками $\Delta_{1}, \ldots, \Delta_{g+1}$ лежит ровно по одной точке $\operatorname{pr} \boldsymbol{\lambda}_{j}$. Значит, дивизор $d=\boldsymbol{\lambda}_{1}+\cdots+\boldsymbol{\lambda}_{g}$ неспециальный, а следовательно, такая проблема обращения Якоби имеет единственной решение. Отсюда вытекает, что $\Psi$-функция, решающая задачу $(\mathscr{R})$, единственна с точностью до нормировки и имеет в бесконечно удаленной точке $\boldsymbol{\lambda}=\infty^{(1)}$ полюс в точности $n$-го порядка. При этом оказывается (подробнее см. $\S 3$, п. 4 ниже), что равномерно внутри области $\widehat{\mathbb{C}} \backslash \widehat{S}$, где $\widehat{S}$ - выпуклая оболочка $S$, справедлива асимптотическая формула

$$
Q_{n}(\lambda)=\Psi\left(\lambda^{(1)}\right)(1+o(1)), \quad n \rightarrow \infty
$$

здесь полиномы $Q_{n}$ нормированы так: старший коэффициент $Q_{n}$ равен старшему (т.е. при $\lambda^{n}$ ) коэффициенту $\Psi$-функции. Такой нормировки $Q_{n}$ мы будем придерживаться всюду в дальнейшем.

Нетрудно видеть, что для любого $\lambda \in \mathbb{C} \backslash S$ выполняется соотношение $\Psi\left(\lambda^{(1)}\right) \Psi\left(\lambda^{(2)}\right) \equiv$ const $\prod_{j=1}^{g}\left(\lambda-\lambda_{j}\right)$, где const $\neq 0$. В дальнейшем мы будем придерживаться следующей нормировки ${ }^{5} \Psi$ -

\footnotetext{
${ }^{5}$ Таким условием $\Psi$-функция определяется однозначно с точностью до знака \pm , выбор знака мы уточним в дальнейшем.
} 
функции:

$$
\Psi\left(\lambda^{(1)}\right) \Psi\left(\lambda^{(2)}\right) \equiv \prod_{j=1}^{g}\left(\lambda-\lambda_{j}\right)
$$

Нетрудно найти и явный вид этой функции. При $\boldsymbol{\lambda} \in \mathfrak{R} \backslash \boldsymbol{\Gamma}$ и в предположении, что $\lambda_{j} \in\left(e_{2 j}, e_{2 j+1}\right)$ имеем:

$$
\Psi(\boldsymbol{\lambda})=\Phi(\boldsymbol{\lambda})^{n-g} e^{A(\boldsymbol{\lambda} ; \rho)} \mathscr{F}_{n}(\boldsymbol{\lambda}) .
$$

Здесь $\Phi(\boldsymbol{\lambda})=e^{G(\boldsymbol{\lambda}, \infty)}-($ многозначная) отображающая функция, $G(\lambda, \infty)=g(\lambda, \infty)+i g^{*}(\lambda, \infty)-$ комплексная функция Грина области $D$,

$$
\begin{aligned}
A(\boldsymbol{\lambda} ; \rho)= & w(\boldsymbol{\lambda})\left[\frac{1}{2 \pi i} \int_{S} \frac{\log \rho(x)}{x-\lambda} \frac{d x}{w^{+}(x)}+\frac{1}{2} c_{g+1}\right. \\
& \left.+\frac{1}{2 \pi i} \sum_{k=1}^{g} v_{k} \int_{\Delta_{k}} \frac{1}{x-\lambda} \frac{d x}{w^{+}(x)}\right],
\end{aligned}
$$

$v_{k}=2 \int_{S} \log \rho(x) d \Omega_{k}^{+}(x)$ (при $g=0 \quad \exp \left\{A\left(\lambda^{(1)} ; \rho\right)\right\}-$ функция Сегё). Функция

$$
\mathscr{F}_{n}(\boldsymbol{\lambda})=\exp \left[\sum_{j=1}^{g} \Omega\left(\boldsymbol{\lambda}_{j}, \infty^{(1)} ; \boldsymbol{\lambda}\right)+2 \pi i \sum_{k=1}^{g} \theta_{k} \Omega_{k}(\boldsymbol{\lambda})\right],
$$

величины $^{6} \theta_{k}=\theta_{k}(n)=\ell_{k}(n)+\left\{(n-g) \omega_{k}(\infty)\right\}$, целые числа $\ell_{k}(n) \in \mathbb{Z}$ равномерно ограничены при $n \rightarrow \infty$, а дивизор $d=$ $\boldsymbol{\lambda}_{1}+\cdots+\boldsymbol{\lambda}_{g}$, где $\boldsymbol{\lambda}_{j}=\boldsymbol{\lambda}_{j}(n)$, является (единственным) решением проблемы обращения Якоби

$$
\begin{aligned}
\sum_{j=1}^{g} \Omega_{k}\left(\boldsymbol{\lambda}_{j}\right) \equiv \frac{i}{\pi} & \int_{S} \log \rho(x) d \Omega_{k}^{+}(x) \\
& -\sum_{j=1}^{g}\left\{\left(n-g+\frac{1}{2}\right) \omega_{j}(\infty)\right\} B_{k j}, \quad k=1, \ldots, g .
\end{aligned}
$$

$\mathrm{B}(31)$ величина $c_{g+1}=c_{g+1}(n), e^{c_{g+1}}=\prod_{j=1}^{g}\left(e_{2 g+2}-\lambda_{j}\right)$. Отметим, что равномерно ограниченные целые числа $\ell_{k}(n), k=1, \ldots, g$,

\footnotetext{
6 Здесь и в (33) $\{\cdot\}$ обозначает дробную часть соответствующего числа.
} 
возникают в (32) в связи с неоднозначностью абелевых интегралов $\Omega_{k}(\boldsymbol{\lambda})$ для $\boldsymbol{\lambda} \in \partial \widetilde{\Re}$ при интегрировании по путям, лежащим в $\widetilde{\mathfrak{R}}, \widetilde{\mathfrak{R}}$ - рассеченная риманова поверхность $\mathfrak{R}$ (подробнее см. приложения А и В). Полином $X_{g}(\lambda):=\prod_{j=1}^{g}\left(\lambda-\lambda_{j}\right)$ является фактически неизвестным "полиномиальным параметром" задачи $(\mathscr{R})$.

Так как функция $\rho$ голоморфна и отлична от нуля на $S$, то правая часть представления (30) имеет смысл как голоморфная функция и при $\boldsymbol{\lambda} \in D^{(1)} \sqcup \boldsymbol{\Gamma}$ для дивизора $d=\boldsymbol{\lambda}_{1}+\cdots+\boldsymbol{\lambda}_{g}$, удовлетворяющего условиям (33). Тем самым, под функцией $\Psi(\boldsymbol{\lambda})$, $\boldsymbol{\lambda} \in D^{(1)} \sqcup \boldsymbol{\Gamma}$, естественно понимать правую часть представления (30). Аналогичное справедливо и для функции $\Psi(\boldsymbol{\lambda})$ при $\boldsymbol{\lambda} \in D^{(2)} \sqcup \boldsymbol{\Gamma}$. На $\boldsymbol{\Gamma}$ эти два голоморфных продолжения не совпадают: для них выполняется краевое условие $3^{\circ}$, где, вообще говоря, функция $\rho \not \equiv 1$.

Вывод явных формул (30)-(33) для $\Psi$-функции дан в приложении В.

\section{§3. Доказательство теоремы 1}

1. В этом пункте мы выведем стандартным образом [22]-[27] сингулярное интегральное уравнение Наттолла (ср. с [28], где подобное уравнение получено несколько иным методом для классического случая $g=0$ и $S=[-1,1])$ для некоторой функции, мероморфной на $\mathfrak{R} \backslash \boldsymbol{\Gamma}$ и связанной с функциями $\Psi, R_{n}$ и $Q_{n}$ (см. ниже формулу (35)), и покажем, что асимптотика свободных нулей $R_{n}$ и блуждающих нулей $Q_{n}$ (т.е. ложных полюсов диагональных аппроксимаций Паде $\left.P_{n} / Q_{n}\right)$ полностью определяется асимптотическим при $n \rightarrow \infty$ поведением точек $\boldsymbol{\lambda}_{1}(n), \ldots, \boldsymbol{\lambda}_{g}(n)$, удовлетворяющих проблеме обращения Якоби (33), а асимптотика самих функций $R_{n}$ и $Q_{n}$ - асимптотическим поведением функции $\Psi(\boldsymbol{\lambda})$ (которая, напомним, зависит от $n$ ).

Непосредственно из определения (5) функции остатка $R_{n}$ и представления (25) получаем, что

$$
R_{n}^{+}(x)-R_{n}^{-}(x)=\frac{2 Q_{n}(x) \rho(x)}{w^{+}(x)}, \quad x \in S \backslash\left\{e_{1}, \ldots, e_{2 g+2}\right\} .
$$

Определим кусочно мероморфную на $\mathfrak{R} \backslash \boldsymbol{\Gamma}$ функцию $F(\boldsymbol{\lambda})$ следующим образом: 


$$
F(\boldsymbol{\lambda})=\Psi(\boldsymbol{\lambda}) \cdot \begin{cases}R_{n}(\lambda) w(\lambda), & \boldsymbol{\lambda} \in D^{(1)}, \\ 2 Q_{n}(\lambda), & \boldsymbol{\lambda} \in D^{(2)},\end{cases}
$$

где функция $\Psi(\boldsymbol{\lambda}), \boldsymbol{\lambda} \in \mathfrak{R} \backslash \boldsymbol{\Gamma}$, определена в (30)-(33). Функция $F$ имеет полюсы только в точках $\infty^{(1)}, \infty^{(2)}$, каждый порядка $g$, а из (34) вытекает, что для скачка $F$ на $\boldsymbol{\Gamma}$ имеем:

$$
F^{+}(\mathbf{x})-F^{-}(\mathbf{x})=V^{-}(\mathbf{x}) \frac{1}{\rho(x)}, \quad \mathbf{x} \in \mathbf{\Gamma},
$$

где функция $V(\boldsymbol{\lambda})=-\Psi(\boldsymbol{\lambda}) R_{n}(\lambda) w(\lambda)=\Psi(\boldsymbol{\lambda}) R_{n}(\lambda) w(\boldsymbol{\lambda}), \boldsymbol{\lambda} \in$ $D^{(2)}$, мероморфна на втором листе. Действительно, положим $\Psi_{1}(\lambda)=\Psi\left(\lambda^{(1)}\right), \Psi_{2}(\lambda)=\Psi\left(\lambda^{(2)}\right), \lambda \in D$. Из краевого условия $3^{\circ}$ задачи $(\mathscr{R})$ вытекает, что $\rho(x) \Psi_{1}^{ \pm}(x)=\Psi_{2}^{\mp}(x)$ при $x \in S$. Умножим обе части $(34)$ на $w^{+}(x) \Psi_{1}^{+}(x)$ и пользуясь последним краевым условием преобразуем (34) к виду:

$$
\begin{aligned}
R_{n}^{+}(x) w^{+}(x) \Psi_{1}^{+}(x)+ & \frac{1}{\rho(x)} R_{n}^{-}(x) w^{-}(x) \Psi_{2}^{-}(x) \\
& =2 Q_{n}(x) \Psi_{2}^{-}(x), \quad x \in S .
\end{aligned}
$$

Аналогично получаем

$$
\begin{aligned}
\frac{1}{\rho(x)} R_{n}^{+}(x) w^{+}(x) \Psi_{2}^{+}(x)+R_{n}^{-}(x) w^{-}(x) \Psi_{1}^{-}(x) & \\
= & 2 Q_{n}(x) \Psi_{2}^{+}(x), \quad x \in S .
\end{aligned}
$$

В совокупности эти соотношения и дают нужные краевые условия для функции $F(\boldsymbol{\lambda})$, заданной равенством (35).

Интегральная формула типа Коши (А.11) для $F$ принимает следующий вид:

$F(\boldsymbol{\lambda})=-\frac{1}{2 \pi i} \int_{\boldsymbol{\Gamma}^{+}} V^{-}(\boldsymbol{\zeta}) \frac{1}{\rho(\zeta)} d \Omega(\boldsymbol{\zeta} ; \boldsymbol{\lambda})+p_{n}(\lambda), \quad \boldsymbol{\lambda} \notin \boldsymbol{\Gamma}, \operatorname{deg} p_{n} \leqslant g$,

где дифференциал $d \Omega(\boldsymbol{\zeta} ; \boldsymbol{\lambda})$ определен (А.10). Рассмотрим эту формулу для $\boldsymbol{\lambda} \in D^{(1)}$. Так как функция $\rho(\zeta)$ голоморфна на каждой связной компоненте $\Delta_{j}$ компакта $S$, то $\rho(\zeta)$ голоморфна на каждой кривой $\boldsymbol{\Gamma}_{j}$. Поэтому контур $\boldsymbol{\Gamma}$ можно не меняя значения интеграла (36) покомпонентно продеформировать в близкий 
контур $\Gamma^{(2)}$, также состоящий из $(g+1)$-й компоненты и целиком расположенный на втором листе $D^{(2)}$ в некоторой окрестности $\boldsymbol{\Gamma}$, в которой голоморфна и отлична от нуля функция $\rho(\zeta)$ (напомним, что функция $V(\boldsymbol{\zeta})$ определена и голоморфна на всем втором листе $\left.D^{(2)}\right)$. Полученная интегральная формула задает голоморфное продолжение функции $F(\boldsymbol{\lambda}), \boldsymbol{\lambda} \in D^{(1)}$, через контур $\boldsymbol{\Gamma}$ на второй лист римановой поверхности вплоть до контура $\Gamma^{(2)}$ (отметим, что для $\boldsymbol{\lambda} \in D^{(2)}$ это продолжение не совпадает с функцией $F(\boldsymbol{\lambda}), \boldsymbol{\lambda} \in D^{(2)}$, определенной (35)). Таким образом, получаем для $\lambda \in D$ :

$$
F\left(\lambda^{(1)}\right)=-\frac{1}{2 \pi i} \int_{\Gamma^{(2)}} V\left(\zeta^{(2)}\right) \frac{1}{\rho(\zeta)} d \Omega\left(\zeta^{(2)} ; \lambda^{(1)}\right)+p_{n}(\lambda) .
$$

Непосредственно из определения функций $F(\boldsymbol{\lambda})$ и $V(\boldsymbol{\lambda})=V\left(\lambda^{(2)}\right)$ и с учетом тождества $\Psi\left(\lambda^{(1)}\right) \Psi\left(\lambda^{(2)}\right)=X_{g}(\lambda)$, для $\boldsymbol{\lambda} \in D^{(2)}$ имеем:

$$
\begin{aligned}
V(\boldsymbol{\lambda}) & =V\left(\lambda^{(2)}\right)=R_{n}(\zeta) w\left(\zeta^{(2)}\right) \Psi\left(\zeta^{(2)}\right) \\
& =-R_{n}(\zeta) w\left(\zeta^{(1)}\right) \Psi\left(\zeta^{(1)}\right) \frac{\Psi\left(\zeta^{(2)}\right)}{\Psi\left(\zeta^{(1)}\right)} \\
& =-\Psi\left(\zeta^{(1)}\right) R_{n}(\zeta) w\left(\zeta^{(1)}\right) \frac{\Psi\left(\zeta^{(1)}\right) \Psi\left(\zeta^{(2)}\right)}{\Psi\left(\zeta^{(1)}\right)^{2}}=-F\left(\zeta^{(1)}\right) \frac{X_{g}(\zeta)}{\Psi\left(\zeta^{(1)}\right)^{2}} .
\end{aligned}
$$

Нетрудно видеть, что операция инволюции $\boldsymbol{\lambda}^{*}=(\lambda, \mp w)$ при $\boldsymbol{\lambda}=$ $(\lambda, \pm w)$ обладает следующим свойством: $d \Omega\left(\boldsymbol{\zeta}^{*}, \boldsymbol{\lambda}\right)=d \Omega\left(\boldsymbol{\zeta}, \boldsymbol{\lambda}^{*}\right)$. Следовательно, $d \Omega\left(\zeta^{(2)}, \lambda^{(1)}\right)=d \Omega\left(\zeta^{(1)}, \lambda^{(2)}\right)$ и с учетом сохранения относительно области $D^{(1)}$ ориентации кривых интегрирования, интегральное представление для $F_{1}(\lambda):=F\left(\lambda^{(1)}\right)=$ $\Psi\left(\lambda^{(1)}\right) R_{n}(\lambda) w\left(\lambda^{(1)}\right)$ и $\lambda \in D$ преобразуется к следующему виду:

$$
\begin{aligned}
F_{1}(\lambda) & =-\frac{1}{2 \pi i} \int_{\Gamma^{(1)}} F\left(\zeta^{(1)}\right) \frac{X_{g}(\zeta)}{\Psi\left(\zeta^{(1)}\right)^{2}} \frac{1}{\rho(\zeta)} d \Omega\left(\zeta^{(1)} ; \lambda^{(2)}\right)+p_{n}(\lambda) \\
& =-\frac{1}{2 \pi i} \int_{\Gamma^{+}} F^{+}\left(\zeta^{(1)}\right) \frac{X_{g}(\zeta)}{\left[\Psi^{+}\left(\zeta^{(1)}\right)\right]^{2}} \frac{1}{\rho(\zeta)} d \Omega^{+}\left(\zeta^{(1)} ; \lambda^{(2)}\right)+p_{n}(\lambda) .
\end{aligned}
$$

Аналогично, для $F_{2}(\lambda):=F\left(\lambda^{(2)}\right)=2 \Psi\left(\lambda^{(2)}\right) Q_{n}(\lambda)$ при $\lambda \in D$ имеем:

$$
F_{2}(\lambda)=-\frac{1}{2 \pi i} \int_{\Gamma^{+}} F^{+}\left(\zeta^{(1)}\right) \frac{X_{g}(\zeta)}{\left[\Psi^{+}\left(\zeta^{(1)}\right)\right]^{2}} \frac{1}{\rho(\zeta)} d \Omega^{+}\left(\zeta^{(1)} ; \lambda^{(1)}\right)+p_{n}(\lambda)
$$


(ср. (37)-(38) с формулой (3.9) из [28], где рассмотрен классический случай $g=0, S=[-1,1])$. Подчеркнем, что эти представления справедливы в общем случае - при любом расположении точек $\boldsymbol{\lambda}_{j}$ на $\mathfrak{R}$. Действительно, при $\boldsymbol{\lambda}_{j} \neq e_{2 j}, e_{2 j+1}$ функции, стоящие под знаком интеграла в (37)-(38), голоморфны на $\boldsymbol{\Gamma}_{j}$ и $\boldsymbol{\Gamma}_{j+1}$ так как функция $\Psi$ не обращается в нуль на этих компонентах $\boldsymbol{\Gamma}$. Если же $\boldsymbol{\lambda}_{j}$ совпадает с концом лакуны, то двукратный нуль в знаменателе, происходящий от функции $\Psi^{2}$, компенсируется нулем функции $F(\boldsymbol{\lambda})$ на $\boldsymbol{\Gamma}$ в точке $\boldsymbol{\lambda}_{j}$ и нулем полинома $X_{g}(\lambda)$.

Формулы (37)-(38) лежат в основе наших последующих рассуждений. Поясним, какого рода информацию мы собираемся из них извлечь. Предположим, например, что по некоторой подпоследовательности $\Lambda \subset \mathbb{N}$ имеем: $\lambda_{j}(n) \Subset\left(e_{2 j}, e_{2 j+1}\right)$ при $n \rightarrow \infty$, $j=1, \ldots, g$. Тогда из (37) мы получим, что для $n \in \Lambda$ асимптотики функции $F_{1}(\lambda):=F\left(\lambda^{(1)}\right)$ и полинома $p_{n}$ в области $D$ совпадают. Функция $F_{1}(\lambda)$ имеет в $D$ нули в тех точках $\lambda_{j}$, для которых $\boldsymbol{\lambda}_{j}=\lambda_{j}^{(1)}$. Следовательно, полином $p_{n}$ обращается в нуль в близких точках. Аналогичный результат вытекает из (38) для полинома $p_{n}$ и функции $F_{2}(\lambda):=F\left(\lambda^{(2)}\right)$. Из определения функций $F_{1}$ и $F_{2}$ вытекает, что их нули, порожденные нулями $\lambda_{1}, \ldots, \boldsymbol{\lambda}_{g}$ функции $\Psi$, различны между собой. Следовательно, для достаточно больших $n \in \Lambda$ полином $p_{n}$, степень которого $\leqslant g$, имеет ровно $g$ нулей в $D$. Тем самым, $\operatorname{deg} p_{n}=g$ и при должной нормировке асимптотика $p_{n}$ совпадает с асимптотикой полинома $\prod_{j=1}^{g}\left(\lambda-\lambda_{j}(n)\right)$. Отсюда уже вытекает асимптотика оставшихся нулей функций $F_{1}$ и $F_{2}$ в $D$. Но их нулями в $D$, отличными от точек $\lambda_{j}$, могут быть только нули $R_{n}$ и $Q_{n}$ соответственно. Тем самым,

$$
Q_{n}(\lambda) R_{n}(\lambda)=\varkappa_{n}^{2}\left(\frac{\prod_{j=1}^{g}\left(\lambda-\lambda_{j}(n)\right)}{\sqrt{h(\lambda)}}+o(1)\right) \text { при } n \rightarrow \infty, n \in \Lambda .
$$

Следовательно,

$$
q_{n}(\lambda) r_{n}(\lambda)=\frac{\prod_{j=1}^{g}\left(\lambda-\lambda_{j}(n)\right)}{\sqrt{h(\lambda)}}+o(1) \text { при } n \rightarrow \infty, n \in \Lambda .
$$

2. Изложим теперь приведенные выше соображения более формально и в полной общности, не ограничиваясь каким-либо частным случаем в поведении точек $\lambda_{j}(n)$. Подчеркнем, что цель 
наших исследований - доказать, что асимптотическое поведение $\boldsymbol{\lambda}_{j}(n)$ как нулей функции $\Psi$ полностъю определяет асимптотическое поведение других возможных нулей функции $F(\boldsymbol{\lambda})$, которыми как следует из (35) могут быть лишь нули функции остатка $R_{n}$ или ортогонального полинома $Q_{n}$.

Для произвольного $\varepsilon>0$ положим

$$
\begin{aligned}
D(\varepsilon) & =\{\lambda \in \widehat{\mathbb{C}}: g(\lambda, \infty)>\varepsilon\}, \\
S(\varepsilon) & =\{\lambda \in \widehat{\mathbb{C}}: g(\lambda, \infty)<\varepsilon\}-\text { окрестность компакта } S, \\
\Gamma(\varepsilon) & =\partial D(\varepsilon)=\{\lambda \in \widehat{\mathbb{C}}: g(\lambda, \infty)=\varepsilon\}, \\
D(0) & =D, \quad S(0)=S .
\end{aligned}
$$

Так как $\rho \neq 0$ и голоморфна на $S$, то $\rho \neq 0$ и голоморфна в $S\left(\varepsilon_{0}\right)$ при некотором $\varepsilon_{0}>0$. В дальнейшем мы рассматриваем только $\varepsilon \in\left(0, \varepsilon_{0}\right)$. Зафиксируем произвольное $\varepsilon \in\left(0, \varepsilon_{0}\right)$ и некоторую последовательность $\left\{\varepsilon_{n}\right\}$ такую, что $\varepsilon_{n} \in(\varepsilon / 3,2 \varepsilon / 3)$ и

$$
\begin{aligned}
& \max _{\lambda \in \Gamma\left(\varepsilon_{n}\right)}\left|p_{n}(\lambda)\right|=O\left(\min _{\lambda \in \Gamma\left(\varepsilon_{n}\right)}\left|p_{n}(\lambda)\right|\right), \\
& \max _{\lambda \in \Gamma\left(\varepsilon_{n}\right)}\left|X_{g}(\lambda)\right|=O\left(\min _{\lambda \in \Gamma\left(\varepsilon_{n}\right)}\left|X_{g}(\lambda)\right|\right)
\end{aligned}
$$

при $n \rightarrow \infty$; тем самым, $X_{g}(\lambda)=\prod_{j=1}^{g}\left(\lambda-\lambda_{j}\right) \neq 0$ и $p_{n}(\lambda) \neq 0$ при $\lambda \in \Gamma\left(\varepsilon_{n}\right)$. Так как функция $F_{1}(\lambda):=F\left(\lambda^{(1)}\right)$ голоморфна в $D \backslash\{\infty\}$ и дифференциал $d \Omega\left(\zeta^{(1)} ; \lambda^{(2)}\right)$ не имеет особенности при $\lambda, \zeta \in D$ (см. (А.12)), то в (37) для $\lambda \in D$ мы можем заменить контур интегрирования $\boldsymbol{\Gamma} \subset \mathfrak{R}$ на контур $\Gamma^{(1)}\left(\varepsilon_{n}\right)$ :

$$
F_{1}(\lambda)=-\frac{1}{2 \pi i} \int_{\Gamma^{(1)}\left(\varepsilon_{n}\right)} F_{1}(\zeta) \frac{X_{g}(\zeta)}{\Psi\left(\zeta^{(1)}\right)^{2}} \frac{1}{\rho(\zeta)} d \Omega\left(\zeta^{(1)} ; \lambda^{(2)}\right)+p_{n}(\lambda),
$$

а формула (40) будет справедлива и для $\lambda \in \Gamma\left(\varepsilon_{n}\right)=\partial D\left(\varepsilon_{n}\right)$. В таком случае учитывая найденное ранее представление для функции $\Psi$ и выбор величин $\varepsilon_{n}$ (см. (39)), получаем, что равномерно по $\lambda \in \Gamma\left(\varepsilon_{n}\right)$ выполняется следующее соотношение

$$
\left|F_{1}(\lambda)-p_{n}(\lambda)\right|=o(1) \cdot \max _{\zeta \in \Gamma\left(\varepsilon_{n}\right)}\left|F_{1}(\zeta)\right|=o(1) \cdot M_{n}
$$


где $M_{n}=\max _{\zeta \in \Gamma\left(\varepsilon_{n}\right)}\left|F_{1}(\zeta)\right|, o(1)=O\left(\delta^{n}\right), \delta=\delta_{n}=e^{-2 \varepsilon_{n}}<e^{-2 \varepsilon / 3}<1$.

Нетрудно видеть, что из (41) вытекает соотношение

$$
m_{n}:=\max _{\lambda \in \Gamma\left(\varepsilon_{n}\right)}\left|p_{n}(\lambda)\right|=M_{n} \cdot(1+o(1))
$$

Тем самым из (41) с учетом (39) получаем

$$
\begin{aligned}
\left|F_{1}(\lambda)-p_{n}(\lambda)\right| & =o(1) \cdot m_{n} \\
& =o(1) \min _{\lambda \in \Gamma\left(\varepsilon_{n}\right)}\left|p_{n}(\lambda)\right|<\left|p_{n}(\lambda)\right|, \quad \lambda \in \Gamma\left(\varepsilon_{n}\right) .
\end{aligned}
$$

Отметим, что непосредственно отсюда по теореме Руше уже вытекает, что для функций $F_{1}$ и $p_{n}$ разность числа их нулей и полюсов в области $D\left(\varepsilon_{n}\right)$ одинакова. Пусть теперь $t=3 \varepsilon / 4>\varepsilon_{n}$,

$$
m_{n}(t)=\max _{\lambda \in \Gamma(t)}\left|p_{n}(\lambda)\right|, \quad M_{n}(t)=\max _{\lambda \in \Gamma(t)}\left|F_{1}(\lambda)\right|
$$

Так как $p_{n}-$ полином степени $\leqslant g$, то $m_{n} \leqslant m_{n}(t)$. Аналитическая функция $\left(F_{1}(\lambda)-p_{n}(\lambda)\right) / \Phi^{g}(\lambda)$ многозначна в $D$, но имеет однозначный модуль (здесь и далее $\Phi(\lambda)=e^{G(\lambda, \infty)}-$ отображающая функция). Поэтому к ней применим принцип максимума модуля. Следовательно,

$$
\begin{aligned}
\max _{\lambda \in \Gamma(t)}\left|\frac{F_{1}(\lambda)-p_{n}(\lambda)}{\Phi^{g}(\lambda)}\right| & \leqslant \max _{\lambda \in \Gamma\left(\varepsilon_{n}\right)}\left|\frac{F_{1}(\lambda)-p_{n}(\lambda)}{\Phi^{g}(\lambda)}\right| \\
& =O(1) \max _{\lambda \in \Gamma\left(\varepsilon_{n}\right)}\left|F_{1}(\lambda)-p_{n}(\lambda)\right| .
\end{aligned}
$$

Тем самым

$$
\max _{\lambda \in \Gamma(t)}\left|F_{1}(\lambda)-p_{n}(\lambda)\right|=o(1) \cdot m_{n}=o(1) \cdot m_{n}(t) .
$$

Отсюда легко вытекает, что $M_{n}(t)=m_{n}(t) \cdot(1+o(1))$.

Заменим теперь в $(38)$ для $F_{2}(\lambda):=F\left(\lambda^{(2)}\right)$ контур интегрирования $\boldsymbol{\Gamma} \subset \mathfrak{R}$ на контур $\Gamma\left(\varepsilon_{n}\right) \subset D$ и рассмотрим эту формулу для $\lambda \in \Gamma(t)$ (напомним, что $\left.t=3 \varepsilon / 4>2 \varepsilon / 3>\varepsilon_{n}\right)$ :

$$
\begin{aligned}
F_{2}(\lambda)=-\frac{1}{2 \pi i} \int_{\Gamma\left(\varepsilon_{n}\right)} & F_{1}(\zeta) \frac{X_{g}(\zeta)}{\Psi\left(\zeta^{(1)}\right)^{2}} \\
\times & \frac{1}{\rho(\zeta)} d \Omega\left(\zeta^{(1)} ; \lambda^{(1)}\right)+p_{n}(\lambda), \quad \lambda \in \Gamma(t) .
\end{aligned}
$$


Тогда, учитывая выбор параметра $t$ и полученные выше соотношения между $M_{n}, m_{n} . M_{n}(t)$ и $m_{n}(t)$, имеем

$$
\max _{\lambda \in \Gamma(t)}\left|F_{2}(\lambda)-p_{n}(\lambda)\right|=o(1) \cdot M_{n}=o(1) \cdot m_{n}=o(1) \cdot m_{n}(t) .
$$

Введем теперь временно новую нормировку: положим $m_{n}(t)=1$ и сохраним прежние обозначения для остальных величин. Тогда из (41) и (43) для многозначных аналитических в $D$ функций $F_{1}(\lambda) / \Phi^{g}(\lambda), F_{2}(\lambda) / \Phi^{g}(\lambda)$ и $p_{n}(\lambda) / \Phi^{g}(\lambda)$, имеющих в $D$ однозначные модули, получаем

$$
\begin{aligned}
& \left|\frac{F_{1}(\lambda)}{\Phi^{g}(\lambda)}-\frac{p_{n}(\lambda)}{\Phi^{g}(\lambda)}\right|=o(1) \quad \text { равномерно по } \lambda \in \overline{D_{t}}, \\
& \left|\frac{F_{2}(\lambda)}{\Phi^{g}(\lambda)}-\frac{p_{n}(\lambda)}{\Phi^{g}(\lambda)}\right|=o(1) \quad \text { равномерно по } \lambda \in \overline{D_{t}}
\end{aligned}
$$

(предполагается, что в левых частях (44) и (45) выбирается одна и та же ветвь многозначной функции $\Phi)$. Рассмотрим последовательность функций $\left\{p_{n} / \Phi^{g}\right\}$. По принципу максимума модуля для аналитических функций с однозначным модулем имеем: $\left|p_{n}(\lambda) / \Phi^{g}(\lambda)\right| \leqslant e^{-t g}$ при $\lambda \in \overline{D_{t}}$ равномерно по $n$ (здесь мы учли новую нормировку $\left.m_{n}(t)=1\right)$. С другой стороны, применяя принцип максимума модуля к полиномам $p_{n}$ в области $S(\tau)$ при произвольном $\tau>t$, получаем: $\max _{\lambda \in \Gamma(\tau)}\left|p_{n}(\lambda)\right|>m_{n}(t)=1$. Тем самым, функция, тождественно равная нулю, не является предельной точкой последовательности $\left\{p_{n} / \Phi^{g}\right\}$ (в топологии равномерной сходимости на компактных подмножествах области $D_{t}$ ). Следовательно, если мы применим к последовательностям функций $F_{1} / \Phi^{g}, F_{2} / \Phi^{g}$ и $p_{n} / \Phi^{g}$ теорему Гурвица, то в силу соотношений (44) и (45) получим, что асимптотическое поведение нулей этих функций, расположенных в области $D_{t}$, одинаково. Так как $|\Phi(\lambda)| \neq 0$ в $D$ и любая однозначная в окрестности точки $\lambda=\infty$ ветвь $\Phi$ имеет полюс первого порядка в точке $\lambda=\infty$, то значит асимптотическое поведение нулей и полюсов функций $F_{1}, F_{2}$ и $p_{n}$, расположенных в области $D_{t}$, также одинаково. Поскольку $\operatorname{deg} p_{n} \leqslant g$, то отсюда, в частности, сразу следует, что функции $F_{1}$ и $F_{2}$ имент в $D_{t}$ не более, чем по $g$ нулей.

Сделаем теперь дальнейшие выводы из того, что функции $F_{1}$ и $F_{2}$ имеют асимптотически одинаковое поведение нулей в $D_{t}$. Из явного вида (35) функции $F(\boldsymbol{\lambda})$ вытекает, что возможные нули 
функции $F_{1}$ - это или точки $\lambda_{j}$ при $\boldsymbol{\lambda}_{j}=\lambda_{j}^{(1)}$, или нули функции остатка $R_{n}$, а возможные нули функции $F_{2}$ - это или точки $\lambda_{j}$ при $\boldsymbol{\lambda}_{j}=\lambda_{j}^{(2)}$, или нули полинома $Q_{n}$. В силу доказанного, и те, и другие точки порождают асимптотически близкие к ним нули полинома $p_{n}$. Если общее число асимптотически различных нулей функций $F_{1}$ и $F_{2}$ равно $g$, то значит имеется ровно $g$ близких к ним нулей полинома $p_{n}$. Так как $\operatorname{deg} p_{n} \leqslant g$, то получаем полное асимптотическое описание полинома $p_{n}, \operatorname{deg} p_{n}=g$, в терминах нулей $F_{1}$ и $F_{2}$.

Предположим теперь, что для некоторой последовательности $\Lambda \subset \mathbb{N}$ имеем: все $\lambda_{j}(n) \Subset\left(e_{2 j}, e_{2 j+1}\right), j=1, \ldots, g, n \in \Lambda$. Так как $t=3 \varepsilon / 4$, а $\varepsilon>0$ произвольно, то для достаточно малого $\varepsilon$ все $\lambda_{j} \in D_{t}$ и попарно различны между собой. Те точки $\lambda_{j}$, для которых $\boldsymbol{\lambda}_{j}=\lambda_{j}^{(1)}$, являются нулями $F_{1}$ и в силу доказанного "притягивают" нули $p_{n}$. Остальные $\lambda_{j}, \boldsymbol{\lambda}_{j}=\lambda_{j}^{(2)},-$ это нули $F_{2}$, которые также "притягивают" нули $p_{n}$. Так как все точки $\lambda_{1}, \ldots, \lambda_{g}$ различны между собой и число их равно $g$, то мы получили полное описание всех нулей $p_{n}$. Точнее, $\operatorname{deg} p_{n}=g$ для всех достаточно больших $n \in \Lambda$ и $\alpha_{n}^{-1} p_{n}(\lambda)=X_{g}(\lambda)+o(1), n \in \Lambda, n \rightarrow \infty$, где $\alpha_{n}$ - старший коэффициент $p_{n}$.

Наконец, рассмотрим случай, когда для некоторого $k \in$ $\{1, \ldots, g\}$ точка $\lambda_{k}(n)$ стремится к концу лакуны: $\lambda_{k}(n) \rightarrow e_{2 k}$ или $\lambda_{k}(n) \rightarrow e_{2 k+1}$ при $n \rightarrow \infty$. Покажем, что и в этом случае $\operatorname{deg} p_{n}=g$ для достаточно больших $n$ и нули $p_{n}$ "притягиваются" точками $\lambda_{1}, \ldots, \lambda_{g}$. Выберем как и выше в представлении (40) для функции $F_{1}(\lambda)$ в качестве $\Gamma^{(1)}$ кривую $\Gamma^{(1)}(\varepsilon)=\{\lambda: g(\lambda, \infty)=\varepsilon\}$, $\varepsilon \in\left(0, \varepsilon_{0}\right)$, и запишем это представление для $\lambda \in D$ в следующем виде (см. (40)):

$$
F_{1}(\lambda)=-\frac{1}{2 \pi i} \int_{\Gamma^{(1)}(\varepsilon)} F_{1}(\zeta) \frac{X_{g}(\zeta)}{\Psi\left(\zeta^{(1)}\right)^{2}} \frac{1}{\rho(\zeta)} d \Omega\left(\zeta^{(1)} ; \lambda^{(2)}\right)+p_{n}(\lambda) .
$$

Будем считать, что этой формулой функция $F_{1}$ задана на $D^{(1)}$, т.е. для $\boldsymbol{\lambda} \in D^{(1)}$ :

$$
F_{1}(\boldsymbol{\lambda})=-\frac{1}{2 \pi i} \int_{\Gamma^{(1)}(\varepsilon)} F_{1}\left(\zeta^{(1)}\right) \frac{X_{g}(\zeta)}{\Psi\left(\zeta^{(1)}\right)^{2}} \frac{1}{\rho(\zeta)} d \Omega\left(\zeta^{(1)} ; \boldsymbol{\lambda}^{*}\right)+p_{n}(\lambda) .
$$

В силу свойств дифференциала $d \Omega(\boldsymbol{\zeta} ; \boldsymbol{\lambda})$, правая часть этого равенства голоморфно продолжается по $\boldsymbol{\lambda}$ на $D^{(2)}$ вплоть до кривой $\Gamma^{(2)}(\varepsilon)$, лежащей на втором листе $D^{(2)}$ "над" кривой $\Gamma(\varepsilon)=$ 
$\Gamma^{(1)}(\varepsilon)$. Тем самым, функция $F_{1}(\boldsymbol{\lambda}), \boldsymbol{\lambda} \in D^{(1)}$, голоморфно продолэсается на второй лист $D^{(2)}$ вплоть до кривой $\Gamma^{(2)}(\varepsilon)$ (это продолжение, вообще говоря, не совпадает с с функцией $F(\boldsymbol{\lambda})$, $\left.\lambda \in D^{(2)}\right)$. С учетом определения функции $F_{1}(\lambda):=F\left(\lambda^{(1)}\right)$ получаем, что на второй лист голоморфно продолжается произведение $\Psi\left(\lambda^{(1)}\right) R_{n}(\lambda) w(\lambda), \lambda \in D$. Обратимся теперь к краевым условиям на функцию $\Psi: \rho(\mathbf{x}) \Psi^{+}(\mathbf{x})=\Psi^{+}(\mathbf{x}), \mathbf{x} \in \mathbf{\Gamma}$. Функция $\rho$ голоморфна и отлична от нуля в некоторой окрестности $S$. Поэтому заменив в этих краевых условиях кривую $\Gamma$ на кривую $\Gamma^{(2)}(\varepsilon)$ при произвольном $\varepsilon \in\left(0, \varepsilon_{0}\right)$, мы, как легко увидеть, получим краевую задачу, решением которой является та же самая функция $\Psi$ (это видно и непосредственно из явных формул (30)-(33)). Таким образом, функция $\Psi\left(\lambda^{(1)}\right), \lambda \in D$, голоморфно продолжается на второй лист вплоть до кривой $\Gamma^{(2)}(\varepsilon)$. Выберем теперь $\varepsilon_{n} \in(\varepsilon / 3,2 \varepsilon / 3)$ так, чтобы при $n \rightarrow \infty$ выполнялись соотношения (39). Рассуждения, вполне аналогичные приведенным выше и опирающиеся на принцип максимума модуля и теорему Гурвица для многозначных аналитических функций в области $D(n)=D^{(1)} \sqcup \boldsymbol{\Gamma} \sqcup S^{(2)}\left(\varepsilon_{n}\right)$ (отметим, что функция $\Phi$ также аналитически продолжается в эту область), показывают, что в этой ситуации имеет место аналог соотношения (44):

$$
\left|\frac{F_{1}(\boldsymbol{\lambda})}{\Phi^{g}(\boldsymbol{\lambda})}-\frac{p_{n}(\boldsymbol{\lambda})}{\Phi^{g}(\boldsymbol{\lambda})}\right|=o(1) \quad \text { равномерно по } \boldsymbol{\lambda} \in \overline{D(n)},
$$

а все нули полинома $p_{n}$ "притягиваются" к точкам $\lambda_{1}, \ldots, \lambda_{n}$ при $n \rightarrow \infty$. Поясним более подробно ту часть рассуждений, которая касается аналитического продолжения функций с первого листа $D^{(1)}$ через кривую $\boldsymbol{\Gamma}$ на второй лист $D^{(2)}$. Предположим для определенности, что $\lambda_{k} \rightarrow e_{2 k}$ при $n \rightarrow \infty$, тем самым, фактически речь идет о продолжении через кривую $\boldsymbol{\Gamma}_{k}$, $\operatorname{pr} \boldsymbol{\Gamma}_{k}=\Delta_{k}=\left[e_{2 k-1}, e_{2 k}\right]$. Будем считать для простоты, что $\Delta_{k}=[-1,1]$. Тогда с помощью функции $u=\lambda+\sqrt{\lambda^{2}-1}$, обратной функции Жуковского, двулистная гиперэллиптическая риманова поверхность $\mathfrak{R}$ конформно отображается на риманову сфеpy $\widehat{\mathbb{C}}$, из которой удалены $2 g$ отрезков вида $\left[\alpha_{j}, \beta_{j}\right],\left[1 / \beta_{j}, 1 / \alpha_{j}\right]$, $j=1, \ldots, g$, где все отрезки $\left[\alpha_{j}, \beta_{j}\right]$ лежат во внешности единичного круга $|u|>1$, а соответствующие им парные отрезки $\left[1 / \beta_{j}, 1 / \alpha_{j}\right]$ - внутри него. При этом верхние берега парных отрезков отождествляются ("склеиваются") между собой, аналогичное 
правило действует и для нижних берегов. При таком отождествлении получаем, очевидно, сферу с $g$ ручками. Первому (открытому) листу $D^{(1)}$ римановой поверхности $\mathfrak{R}$ соответствует внешность единичного круга $|u|>1$ с разрезами по отрезкам $\left[\alpha_{j}, \beta_{j}\right]$, $j=1, \ldots, g$, второму листу $D^{(2)}$ - внутренность единичного круга $|u|<1$ с разрезами по отрезкам $\left[1 / \beta_{j}, 1 / \alpha_{j}\right], j=1, \ldots, g$; см. рис. 1. Обратное отображение с помощью функции Жуковского $\lambda=\frac{1}{2}(u+1 / u)$ задает параметрическое представление $\mathfrak{R}$ и делает очевидным процесс аналитического продолжения через кривую $\boldsymbol{\Gamma}_{k}=\{\lambda(u):|u|=1\}$ с первого листа римановой поверхности на второй. Действительно, для весовой функции $\rho$ имеем: $\rho(\lambda)=\rho\left(\frac{1}{2}(u+1 / u)\right)$, для полинома $p_{n}: p_{n}(\lambda)=p_{n}\left(\frac{1}{2}(u+1 / u)\right)$. Отметим, что из такого симметричного представления вытекает, что каждой точке $\lambda_{k} \rightarrow e_{2 k}$ соответствуют два близких нуля продолженной функции $p_{n}\left(\frac{1}{2}(u+1 / u)\right)$. Однако никакого противоречия в этом нет, так как это означает, что продолженная на второй лист функция $F_{1}(\boldsymbol{\lambda})$ также имеет в окрестности точки $e_{2 k}$ два нуля: один нуль происходит от функции $\Psi(\boldsymbol{\lambda})$, а второй - от функции остатка $R_{n}(\lambda)$ (см. (35)).

Итак, мы показали, что в общей ситуации $\operatorname{deg} p_{n}=g$ для достаточно больших $n$ и асимптотика $g$ нулей полинома $p_{n}$ полностью определяется асимптотическим поведением точек $\lambda_{1}, \ldots, \lambda_{n}$, точнее,

$$
\alpha_{n}^{-1} p_{n}(\lambda)=X_{g}(\lambda)+o(1), \quad n \rightarrow \infty
$$

где $\alpha_{n}$ - старший коэффициент $p_{n}$. С учетом последнего равенства нетрудно видеть, что полученные выше соотношения (44)(45) сохранятся, если вместо нормировки $m_{n}(t)=1$ мы будем считать, что старший коэффициент полинома $p_{n}$ равен 2 : $p_{n}(\lambda)=2 \lambda^{g}+\cdots$. Тем самым,

$$
\begin{aligned}
& p_{n}(\lambda)=2 X_{g}(\lambda)+o(1), \quad n \rightarrow \infty, \text { равномерно внутри } \mathbb{C}, \\
& p_{n}(\lambda)=2 X_{g}(\lambda)(1+o(1)), \quad n \rightarrow \infty, \text { равномерно на } \Gamma\left(\varepsilon_{n}\right) .
\end{aligned}
$$

Следовательно, во всех полученных выше соотношениях $p_{n}$ можно заменить на $2 X_{g}$. Кроме того, как нетрудно видеть, в этих соотношениях величина $o(1)=o\left(\delta^{n}\right)$, где $\delta \in(0,1)$ и зависит от $\rho$.

3. С помощью соотношений (44)-(45) и (48) уже легко завершается доказательство теоремы 1. Действительно, в силу опреде- 


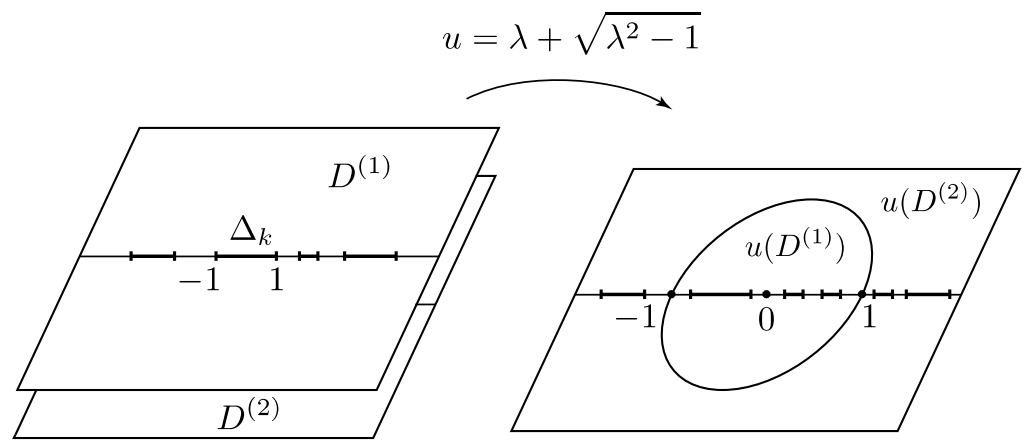

Рис. 1. При $\Delta_{k}=[-1,1]$ с помощью функции $u=\lambda+\sqrt{\lambda^{2}-1}$, обратной функции Жуковского, двулистная гиперэллиптическая риманова поверхность $\mathfrak{R}$ конформно отображается на риманову сферу $\widehat{\mathbb{C}}$, из которой удалены $2 g$ отрезков вида $\left[\alpha_{j}, \beta_{j}\right]$, $\left[1 / \beta_{j}, 1 / \alpha_{j}\right], j=1, \ldots, g$, где все отрезки $\left[\alpha_{j}, \beta_{j}\right]$ лежат во внешности единичного круга $|u|>1$, а соответствующие им парные отрезки $\left[1 / \beta_{j}, 1 / \alpha_{j}\right]-$ внутри него. При этом верхние берега парных отрезков отождествляются ("склеиваются") между собой, аналогичное правило действует и для нижних берегов.

ления функций $F_{1}$ и $F_{2}$ из $(44)-(45)$ и (48) получаем:

$$
\begin{aligned}
\Psi\left(\lambda^{(1)}\right) R_{n}(\lambda) w(\lambda) & =2 X_{g}(\lambda)(1+o(1)) \quad \text { равномерно на } \Gamma\left(\varepsilon_{n}\right), \\
\Psi\left(\lambda^{(2)}\right) Q_{n}(\lambda) & =X_{g}(\lambda)(1+o(1)) \quad \text { равномерно на } \Gamma\left(\varepsilon_{n}\right) .
\end{aligned}
$$

Перемножая теперь левые и правые части этих соотношений и пользуясь тем, что $\Psi\left(\lambda^{(1)}\right) \Psi\left(\lambda^{(2)}\right)=X_{g}(\lambda)$, получим

$$
\begin{aligned}
Q_{n}(\lambda) R_{n}(\lambda) & =\frac{2 X_{g}(\lambda)}{w(\lambda)}(1+o(1)) \\
& =\frac{2 X_{g}(\lambda)}{w(\lambda)}+o(1) \quad \text { равномерно на } \Gamma\left(\varepsilon_{n}\right) .
\end{aligned}
$$

Но $\varepsilon_{n} \in(\varepsilon / 3,2 \varepsilon / 3)$, тем самым, равномерно в $\overline{D(\varepsilon)}=\{\lambda$ : $g(\lambda, \infty) \geqslant \varepsilon\}$ имеем:

$$
Q_{n}(\lambda) R_{n}(\lambda)=\frac{2 X_{g}(\lambda)}{w(\lambda)}+o(1), \quad n \rightarrow \infty .
$$


Отсюда, так как

$$
Q_{n}(\lambda) R_{n}(\lambda)=\frac{1}{\pi} \int_{S} \frac{Q_{n}^{2}(x)}{\lambda-x} \frac{\rho(x) d x}{\sqrt{-h(x)}}
$$

вытекает, что

$$
\frac{1}{\pi} \int_{S} \frac{Q_{n}^{2}(x) \rho(x) d x}{\sqrt{-h(x)}}=2+o(1), \quad n \rightarrow \infty
$$

Тем самым, окончательно имеем:

$$
q_{n}(\lambda) r_{n}(\lambda)=\frac{X_{g}(\lambda)}{\sqrt{h(\lambda)}}+o\left(\delta^{n}\right), \quad n \rightarrow \infty, \delta \in(0,1),
$$

равномерно в $\overline{D(\varepsilon)}=\{\lambda: g(\lambda, \infty) \geqslant \varepsilon\}$. Поскольку $\varepsilon>0-$ произвольно, теорема 1 доказана.

4. При подходящей нормировке полиномов $Q_{n}(\lambda)$ - знаменателей диагональных аппроксимаций Паде функции $\widehat{\mu}-$ их сильная асимптотика внутри области $\widehat{\mathbb{C}} \backslash \widehat{S}$ и на компакте $S$ описывается в терминах $\Psi$-функции, решающей задачу $(\mathscr{R})$, следующим образом.

ПРЕДЛОЖЕНИЕ. Пусть голоморфная на $S$ функиия $\rho$ удовле-

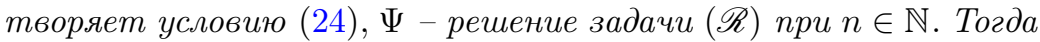
при подходящей нормировке полиномов $Q_{n}(\lambda)$ имеем:

$1^{\circ} . Q_{n}(\lambda)=\Psi(\lambda)\left(1+o\left(\delta^{n}\right)\right)$ nри $n \rightarrow \infty$ равномерно внутри $\widehat{\mathbb{C}} \backslash \widehat{S}$;

$2^{\circ} \cdot Q_{n}(x) X_{g}(x)=\left(\Psi^{+}(x)+\Psi^{-}(x)\right) X_{g}(x)+o\left(\delta^{n}\right)$ nри $n \rightarrow \infty$ равномерно на $S$.

Здесь $\Psi(\lambda)=\Psi\left(\lambda^{(1)}\right)$, под $\Psi^{+}(x)\left(\Psi^{-}(x)\right)$ понимаются верхние (соответственно, нижние) предельные значения $\Psi(\lambda)$ на $S$. Функция $\Psi$, решающая задачу $(\mathscr{R})$, имеет в точке $\boldsymbol{\lambda}=\infty^{(1)}$ полюс в точности $n$-го порядка. Так как $\operatorname{deg} Q_{n}=n$ при всех $n$ и $\Psi\left(\lambda^{(1)}\right) / \lambda^{n} \rightarrow \varkappa_{n} \neq 0$ при $\lambda \rightarrow \infty$, где $\varkappa_{n} \in \mathbb{R}$, то естественно нормировать $Q_{n}$ условием: $Q_{n}(\lambda)=\varkappa_{n} \lambda^{n}+\cdots$. С помощью представления (30)-(33) величину $\varkappa_{n}$ нетрудно найти и в явном виде (см. приложение В), при этом, так как $\varkappa_{n} \in \mathbb{R}$, можно считать, что $\varkappa_{n}>0$; таким выбором знака у $\varkappa_{n}$ однозначно определяется и сама $\Psi$-функция. 
ДокАЗАТЕЛЬСтво. Пункт $1^{\circ}$ предложения вытекает непосредственно из соотношения (49) и равенства $\Psi\left(\lambda^{(1)}\right) \Psi\left(\lambda^{(2)}\right)=$ $X_{g}(\lambda)$.

Для доказательства п. $2^{\circ}$ поступим следующим образом. Из (47) и (48) вытекает, что равномерно на $\boldsymbol{\Gamma}$ имеем: $F_{1}(\boldsymbol{\lambda})=$ $2 X_{g}(\lambda)+o(1), n \rightarrow \infty, o(1)=o\left(\delta^{n}\right)$. Пользуясь определением функций $F_{1}$ и $F$ перепишем последнее соотношение в виде двух равенств для $x \in S$ следующим образом:

$$
\begin{aligned}
& \Psi^{+}(x) R_{n}^{+}(x) w^{+}(x)=2 X_{g}(x)+o(1), \\
& \Psi^{-}(x) R_{n}^{-}(x) w^{-}(x)=2 X_{g}(x)+o(1) .
\end{aligned}
$$

Умножим обе части первого равенства на $\Psi^{-}(x)$, а второго - на $\Psi^{+}(x)$, сложим получившиеся соотношения и воспользуемся равенствами (34) и $\rho(x) \Psi^{+}(x) \Psi^{-}(x)=X_{g}(x)$. Получим

$$
Q_{n}(x) X_{g}(x)=\left(\Psi^{+}(x)+\Psi^{-}(x)\right) X_{g}(x)+o(1),
$$

что и требовалось доказать.

\section{§4. Доказательство теоремы 2}

1. Доказательство первой части теоремы 2 - формулы (28) - не составляет труда и следует непосредственно из теоремы 1 . Действительно, с одной стороны

$$
q_{n}(\lambda) r_{n}(\lambda)=\frac{1}{\pi} \int_{S} \frac{q_{n}^{2}(x)}{\lambda-x} \frac{\rho(x) d x}{\sqrt{-h(x)}},
$$

с другой

$$
\begin{aligned}
q_{n}(\lambda) r_{n}(\lambda)= & \frac{X_{g}(\lambda)}{\sqrt{h(\lambda)}}+o\left(\delta^{n}\right), \\
& n \rightarrow \infty, \delta \in(0,1), \text { равномерно в } \overline{D(\varepsilon)},
\end{aligned}
$$

где $\overline{D(\varepsilon)}=\{\lambda: g(\lambda, \infty) \geqslant \varepsilon\}, \varepsilon>0-$ произвольно. Пользуясь (51) разложим функцию $q_{n}(\lambda) r_{n}(\lambda)$ в ряд по степеням $1 / \lambda$ в бесконечно удаленной точке:

$$
q_{n}(\lambda) r_{n}(\lambda)=\frac{d_{0}}{\lambda}+\frac{d_{1}}{\lambda^{2}}+\cdots,
$$


где

$$
d_{0}=\frac{1}{\pi} \int_{S} \frac{q_{n}^{2}(x) \rho(x) d x}{\sqrt{-h(x)}}=1, \quad d_{1}=\frac{1}{\pi} \int_{S} \frac{x q_{n}^{2}(x) \rho(x) d x}{\sqrt{-h(x)}} .
$$

Непосредственно из рекуррентной формулы (9) сдедует (см. (11)), что $d_{1}=b_{n+1}$. В силу (52) коэффициенты $d_{0}, d_{1}, \ldots$ с точностью до $o\left(\delta^{n}\right)$ совпадают с соответствующими лорановскими коэффициентами $c_{0}, c_{1}, \ldots$ функции $X_{g}(\lambda) / \sqrt{h(\lambda)}$. Так как $c_{0}=1$, то

$$
c_{1}=\lim _{\lambda \rightarrow \infty} \lambda\left(\frac{\lambda X_{g}(\lambda)}{\sqrt{h(\lambda)}}-1\right)=\lim _{\lambda \rightarrow \infty} \lambda\left(\frac{\lambda X_{g}(\lambda)-\sqrt{h(\lambda)}}{\lambda^{g}}\right) .
$$

Прямые вычисления дают для этой величины следующее представление:

$$
c_{1}=\frac{1}{2} \sum_{j=1}^{2 g+2} e_{j}-\sum_{j=1}^{g} \lambda_{j}(n)
$$

Тем самым,

$$
b_{n+1}=\frac{1}{2} \sum_{j=1}^{2 g+2} e_{j}-\sum_{j=1}^{g} \lambda_{j}(n)+o\left(\delta^{n}\right), \quad n \rightarrow \infty .
$$

Формула (28) доказана.

2. Для доказательства (29) поступим следующим образом. Из рекуррентной формулы (9) вытекает (см. (11)), что

$$
a_{n}=\frac{1}{\pi} \int_{S} x q_{n-1}(x) q_{n}(x) \frac{\rho(x) d x}{\sqrt{-h(x)}}=\frac{k_{n-1}}{k_{n}},
$$

$k_{n}>0$ - старший коэффициент ортонормированного полинома $q_{n}$. Из (50) получаем, что $k_{n}$ и $\varkappa_{n}>0$ - старший коэффициент полинома $Q_{n}$ - связаны соотношением $\varkappa_{n}=k_{n}(\sqrt{2}+o(1))$, где $o(1)=o\left(\delta^{n}\right)$. Следовательно, $a_{n}=\frac{\varkappa_{n-1}}{\varkappa_{n}}(1+o(1))$. Но величина $\varkappa_{n}$ связана с нормировкой $\Psi$-функции: $\Psi(\lambda)=\varkappa_{n} \lambda^{n}+\cdots$ при $\lambda \rightarrow$ $\infty$. Пользуясь явным представлением (30)-(33) для $\Psi$-функции найдем теперь асимптотику отношения величин $\varkappa_{n-1}$ и $\varkappa_{n}$ при $n \rightarrow \infty$.

Tак как $\Psi\left(\lambda^{(1)}\right) \Psi\left(\lambda^{(2)}\right) \equiv \prod_{j=1}^{g}\left(\lambda-\lambda_{j}\right)$, то

$$
\Psi\left(\lambda^{(2)}\right)=\frac{1}{\varkappa_{n} \lambda^{n-g}}+\cdots, \quad \lambda \rightarrow \infty .
$$


Следовательно,

$$
\frac{\Psi\left(\lambda^{(1)}\right)}{\Psi\left(\lambda^{(2)}\right)}=\varkappa_{n}^{2} \lambda^{2 n-g}+\cdots, \quad \lambda \rightarrow \infty,
$$

и

$$
\varkappa_{n}^{2}=\lim _{\lambda \rightarrow \infty} \frac{\Psi\left(\lambda^{(1)}\right)}{\Psi\left(\lambda^{(2)}\right)} \lambda^{g-2 n},
$$

при этом можно считать, что $\lambda>0$. C помощью явных формул (30)-(33) получаем

$$
\frac{\Psi\left(\lambda^{(1)}\right)}{\Psi\left(\lambda^{(2)}\right)}=\Phi^{2 n-2 g} e^{A\left(\lambda^{(1)} ; \rho\right)-A\left(\lambda^{(2)} ; \rho\right)} \frac{\mathscr{F}_{n}\left(\lambda^{(1)}\right)}{\mathscr{F}_{n}\left(\lambda^{(2)}\right)},
$$

где первый сомножитель ведет себя в бесконечности как

$$
(\operatorname{cap} S)^{2 g-2 n} \lambda^{2 n-2 g}+\cdots,
$$

а второй не зависит от $n$. Тем самым,

$$
\frac{\varkappa_{n-1}^{2}}{\varkappa_{n}^{2}}=\lim _{\lambda \rightarrow \infty}\left[\frac{\lambda^{2}}{\Phi^{2}(\lambda)} \cdot \frac{\mathscr{F}_{n-1}\left(\lambda^{(1)}\right)}{\mathscr{F}_{n-1}\left(\lambda^{(2)}\right)} \cdot \frac{\mathscr{F}_{n}\left(\lambda^{(2)}\right)}{\mathscr{F}_{n}\left(\lambda^{(1)}\right)}\right] .
$$

Преобразуем теперь величину $\mathscr{F}_{n}\left(\lambda^{(1)}\right) / \mathscr{F}_{n}\left(\lambda^{(2)}\right)=\exp \left(\varphi_{n}(\lambda)\right)$, где (см. (32))

$$
\begin{gathered}
\varphi_{n}(\lambda)=\sum_{j=1}^{g}\left[\Omega\left(\boldsymbol{\lambda}_{j}(n), \infty^{(1)} ; \lambda^{(1)}\right)-\Omega\left(\boldsymbol{\lambda}_{j}(n), \infty^{(1)} ; \lambda^{(2)}\right)\right] \\
+2 \pi i \sum_{j=1}^{g} \theta_{j}(n)\left(\Omega_{j}\left(\lambda^{(1)}\right)-\Omega_{j}\left(\lambda^{(2)}\right)\right) .
\end{gathered}
$$

По правилу (А.7) перестановки пределов интегрирования и параметров для $a$-нормированных абелевых дифференциалов имеем:

$$
\begin{aligned}
\Omega\left(\boldsymbol{\lambda}_{j}, \infty^{(1)} ; \lambda^{(1)}\right)-\Omega\left(\boldsymbol{\lambda}_{j}, \infty^{(1)} ; \lambda^{(2)}\right) \\
=-\int_{\lambda^{(1)}}^{\lambda^{(2)}} d \Omega\left(\boldsymbol{\lambda}_{j}, \infty^{(1)} ; \boldsymbol{\zeta}\right) \\
=-\int_{\boldsymbol{\lambda}_{j}}^{\infty^{(1)}} d \Omega\left(\lambda^{(1)}, \lambda^{(2)} ; \boldsymbol{\zeta}\right)(\bmod 2 \pi i) \\
=\int_{\infty^{(1)}}^{\boldsymbol{\lambda}_{j}} d \Omega\left(\lambda^{(1)}, \lambda^{(2)} ; \boldsymbol{\zeta}\right)(\bmod 2 \pi i) .
\end{aligned}
$$


Так как при $x \in \mathbb{R} \backslash S$

$$
d G\left(x^{(1)}, x^{(2)}, \boldsymbol{\zeta}\right)+2 \pi i \sum_{j=1}^{g} \omega_{j}(x) d \Omega_{j}(\boldsymbol{\zeta})+d \Omega\left(x^{(1)}, x^{(2)}, \boldsymbol{\zeta}\right)=0
$$

(см. (А.6)), то при $\lambda>e_{2 g+2}$

$$
\begin{aligned}
\int_{\infty^{(1)}}^{\boldsymbol{\lambda}_{j}} d \Omega\left(\lambda^{(1)}, \lambda^{(2)} ; \boldsymbol{\zeta}\right) \\
=-\int_{\infty^{(1)}}^{\boldsymbol{\lambda}_{j}} d G\left(\lambda^{(1)}, \lambda^{(2)} ; \boldsymbol{\zeta}\right)-2 \pi i \sum_{k=1}^{g} \omega_{k}(\lambda) \int_{\infty^{(1)}}^{\boldsymbol{\lambda}_{j}} d \Omega_{k}(\boldsymbol{\zeta}) \\
=-\left(g\left(\boldsymbol{\lambda}_{j}, \lambda\right)-g\left(\infty^{(1)}, \lambda\right)\right)+i \beta_{j}(n) \\
\quad-2 \pi i \sum_{k=1}^{g} \omega_{k}(\lambda)\left(\Omega_{k}\left(\boldsymbol{\lambda}_{j}\right)-\Omega_{k}\left(\infty^{(1)}\right)\right)
\end{aligned}
$$

где $\beta_{j}(n) \in \mathbb{R}$. Воспользуемся теперь тем, что $\Omega_{k}\left(\lambda^{(1)}\right)-\Omega_{k}\left(\lambda^{(2)}\right)=$ $2 \Omega_{k}\left(\lambda^{(1)}\right)$, и формулой (В.12). Тогда из $(56),(57)$ и (В.12) получим

$$
\begin{aligned}
\varphi_{n}(\lambda)=- & \sum_{j=1}^{g}\left[g\left(\boldsymbol{\lambda}_{j}, \lambda\right)-g\left(\infty^{(1)}, \lambda\right)\right] \\
& +2 \pi i \sum_{k=1}^{g} \omega_{k}(\lambda)\left(\sum_{j=1}^{g} \theta_{j}(n) B_{k j}\right) \\
& +2 \pi i \sum_{j=1}^{g} \theta_{j}(n) \cdot 2 \Omega_{j}\left(\lambda^{(1)}\right)+i \beta(n)+C_{1}(\lambda),
\end{aligned}
$$

где $\beta(n) \in \mathbb{R}, \theta_{j}=\theta_{j}(n)=\ell_{j}(n)+\left\{(n-g) \omega_{j}(\infty)\right\}$, величины $\ell_{j}(n) \in$ $\mathbb{Z}$ и равномерно ограничены при $n \rightarrow \infty$, а $C_{1}(\lambda)$ не зависит от $n$. Поменяем в (58) порядок суммирования во втором слагаемом и воспользуемся тем, что (см. (А.8))

$$
\sum_{k=1}^{g} \omega_{k}(\lambda) B_{k j}=-2 i \operatorname{Im} \Omega_{j}\left(\lambda^{(1)}\right), \quad \lambda \in D .
$$

Тогда это второе слагаемое примет вид:

$$
2 \pi i \sum_{k=1}^{g} \omega_{k}(\lambda)\left(\sum_{j=1}^{g} \theta_{j} B_{k j}\right)=2 \pi i \sum_{j=1}^{g} \theta_{j}\left(\sum_{k=1}^{g} \omega_{k}(\lambda) B_{k j}\right)=
$$




$$
=2 \pi i \sum_{j=1}^{g} \theta_{j}\left(-2 i \operatorname{Im} \Omega_{j}\left(\lambda^{(1)}\right)\right)=-2 \pi i \sum_{j=1}^{g} \theta_{j}\left(2 i \operatorname{Im} \Omega_{j}\left(\lambda^{(1)}\right)\right) .
$$

Следовательно, из (58) имеем:

$$
\varphi_{n}(\lambda)=-\sum_{j=1}^{g} g\left(\boldsymbol{\lambda}_{j}, \lambda\right)+i \beta(n)+C_{2}(\lambda)
$$

где $\beta(n) \in \mathbb{R}$, a $C_{2}(\lambda)$ не зависит от $n$. Окончательно из последней формулы и (55) получаем:

$$
\frac{\varkappa_{n-1}^{2}}{\varkappa_{n}^{2}}=(\operatorname{cap} S)^{2} \exp \left\{\sum_{j=1}^{g} g\left(\boldsymbol{\lambda}_{j}(n), \infty\right)-\sum_{j=1}^{g} g\left(\boldsymbol{\lambda}_{j}(n-1), \infty\right)\right\},
$$

тем самым,

$$
\begin{aligned}
a_{n}= & \frac{\varkappa_{n-1}}{\varkappa_{n}}\left(1+o\left(\delta^{n}\right)\right) \\
= & \operatorname{cap} S \cdot \exp \left\{\frac{1}{2} \sum_{j=1}^{g} g\left(\boldsymbol{\lambda}_{j}(n), \infty\right)-\frac{1}{2} \sum_{j=1}^{g} g\left(\boldsymbol{\lambda}_{j}(n-1), \infty\right)\right\} \\
& \quad \times\left(1+o\left(\delta^{n}\right)\right) .
\end{aligned}
$$

Асимптотическая формула (29), а вместе с ней и теорема 2, доказаны.

\section{Приложение A}

1. Приведем некоторые необходимые нам стандартные сведения о гиперэллиптических римановых поверхностях. В обозначениях и терминологии мы здесь придерживаемся в основном [31] (см. также [32]-[33]).

Пусть $\mathfrak{R}$ - гиперэллиптическая риманова поверхность рода $g \geqslant 1$, заданная уравнением $w^{2}=h(\lambda)$, где $h(\lambda)=\prod_{j=1}^{2 g+2}\left(\lambda-e_{j}\right)$, $e_{1}<\cdots<e_{2 g+2}$. Будем считать, что $\mathfrak{R}$ реализована как двулистное разветвленное в точках $\left\{e_{j}\right\}$ накрытие римановой сферы $\widehat{\mathbb{C}}$ таким образом, что переход с одного листа на другой осуществляется по верхнему $\Delta_{j}^{+}$и нижнему $\Delta_{j}^{-}$берегам отрезков 
$\Delta_{j}=\left[e_{2 j-1}, e_{2 j}\right], j=1, \ldots, g+1$. Тем самым, над каждой точкой $\widehat{\mathbb{C}}$ за исключением точек ветвления $\left\{e_{j}\right\}$ лежат ровно две точки римановой поверхности, а каждому отрезку $\Delta_{j}$ соответствует на $\mathfrak{R}$ замкнутая аналитическая (в комплексной структуре $\mathfrak{R}$ ) кривая $\boldsymbol{\Gamma}_{j}, j=1, \ldots, g+1,-$ цикл на $\mathfrak{R} ;$ положим $\boldsymbol{\Gamma}=\bigcup_{j=1}^{g+1} \boldsymbol{\Gamma}_{j}$. Выберем в $D=\widehat{\mathbb{C}} \backslash S$ ту ветвь квадратного корня, которая положительна при положительных значениях аргумента. Тем самым, $\sqrt{h(\lambda)} / \lambda^{g+1} \rightarrow 1$ при $\lambda \rightarrow \infty$. Функция $w: w^{2}=h(\lambda)$ однозначна на $\mathfrak{R}$. Первым (открытым) листом $D^{(1)}$ поверхности $\mathfrak{R}$ будем считать тот, на котором $w=\sqrt{h(\lambda)}$. На втором листе $D^{(2)}$ имеем: $w=-\sqrt{h(\lambda)}$. Для точек римановой поверхности $\mathfrak{R}$ будем использовать обозначение $\boldsymbol{\lambda}=(\lambda, w)$, где $w= \pm \sqrt{h(\lambda)}$; при этом для точек первого листа $\lambda^{(1)}=(\lambda, \sqrt{h(\lambda)})$, а для точек второго $\lambda^{(2)}=(\lambda,-\sqrt{h(\lambda)})$. Вместо $\boldsymbol{\lambda}=(\lambda, \pm \sqrt{h(\lambda)})$ иногда мы будем писать коротко $\boldsymbol{\lambda}=(\lambda, \pm)$. Область $D^{(1)}$ будем как правило отождествлять с "физической" областью $D$. Для $\boldsymbol{\lambda}=\lambda^{(1)}$ будем часто писать просто $w(\lambda)$ вместо $w(\boldsymbol{\lambda})$; тем самым, приобретает смысл и запись $w^{ \pm}(x)=\sqrt{h(x \pm i 0)}, x \in S$. Каноническая проекция $\operatorname{pr}: \mathfrak{R} \rightarrow \widehat{\mathbb{C}}$ определяется соотношением pr $\boldsymbol{\lambda}=\lambda$, в частности $\operatorname{pr} D^{(1)}=\operatorname{pr} D^{(2)}=D, \operatorname{pr} \boldsymbol{\Gamma}=S$. Замкнутые циклы на $\mathfrak{R}$, соответствующие замкнутым лакунам $L_{j}=\left[e_{2 j}, e_{2 j+1}\right], j=1, \ldots, g$, будем обозначать через $\mathbf{L}_{j}$. Тем самым $\operatorname{pr} \mathbf{L}_{j}=\left[e_{2 j}, e_{2 j+1}\right]$.

Ориентируем кривые $\boldsymbol{\Gamma}_{j}$ так, что при обходе по $\boldsymbol{\Gamma}_{j}$ область $D^{(1)}$ остается слева, а $D^{(2)}$ - справа. Примем ориентированные кривые $\boldsymbol{\Gamma}_{j}, j=1, \ldots, g$, за $a$-циклы $\mathbf{a}_{j}$ на $\mathfrak{R}$ и стандартным образом [31], [32] дополним их $b$-циклами $\mathbf{b}_{j}$ до базиса гомологий $\left\{\mathbf{a}_{j}, \mathbf{b}_{j}\right\}_{j=1, \ldots, g}$ на $\mathfrak{R}$. Пусть $\left\{d \Omega_{k}\right\}$ - соответствующий $a-$ нормированный базис голоморфных абелевых дифференциалов: $\oint_{\mathbf{a}_{j}} d \Omega_{k}=\delta_{k j}, k, j=1, \ldots, g$. Матрица $\mathbf{B}=\left\|B_{k j}\right\|_{k, j=1, \ldots, g}$, где $B_{k j}=\oint_{\mathbf{b}_{j}} d \Omega_{k}-b$-периоды базисных дифференциалов, является матрицей Римана: она симметрична, $B_{k j}=B_{j k}$, а ее мнимая часть положительно определена, $\left\|\operatorname{Im} B_{j k}\right\|>0$. В рассматриваемом здесь нами случае, когда все точки $e_{j} \in \mathbb{R}$, все величины $B_{k j}$ - чисто мнимые. Абелевы интегралы $\Omega_{k}(\boldsymbol{\lambda})=\int_{e_{2 g+2}}^{\boldsymbol{\lambda}} d \Omega_{k}$ определены на $\mathfrak{R}$ однозначно по модулю их $a$ и $b$-периодов. Так как $\Omega_{k}^{\prime}(\lambda)=s_{k}(\lambda) / w(\lambda)$, где $s_{k}$ - полином степени $g-1$, то имеет смысл обозначение $d \Omega_{k}^{ \pm}(x)=s_{k}(x) d x / w^{ \pm}(x)$ при $x \in S$. 
Если на $\mathfrak{R} \backslash \boldsymbol{\Gamma}$ задана функция $F(\boldsymbol{\lambda})$, то под $F^{+}(\mathbf{x})$ понимаются ее предельные значения при $\boldsymbol{\lambda} \rightarrow \mathbf{x} \in \boldsymbol{\Gamma}, \boldsymbol{\lambda} \in D^{(1)}$, если они существуют; аналогичный смысл придается и $F^{-}(\mathbf{x})$ при $\boldsymbol{\lambda} \in D^{(2)}$, $\boldsymbol{\lambda} \rightarrow \mathrm{x} \in \boldsymbol{\Gamma}$.

Фиксируем стандартный базис $\mathbf{e}_{1}, \mathbf{e}_{2}, \ldots, \mathbf{e}_{g}$ в $\mathbb{R}^{g}:\left(\mathbf{e}_{k}\right)_{j}=\delta_{k j}$. Тогда векторы $\mathbf{e}_{1}, \ldots, \mathbf{e}_{g}, \mathbf{B e}_{1}, \ldots, \mathbf{B e}_{g}$ линейно независимы над $\mathbb{R}$ и образуют базис в $\mathbb{C}^{g}$. Факторпространство $\mathbb{C}^{g} /\{\mathrm{N}+\mathrm{BM}\}$ по целочисленной решетке, $\mathrm{N}, \mathrm{M} \in \mathbb{Z}^{g}$, является $2 g$-мерным вещественным тором $\mathbb{T}^{2 g}$ и называется многообразием Якоби Јас $\Re$ римановой поверхности $\mathfrak{R}$. Любой вектор $\mathbf{v} \in \mathbb{C}^{g}$ однозначно представим в виде $\mathbf{v}=\mathbf{x}+\mathbf{B y}+\mathbf{N}+\mathbf{B M}=\mathbf{x}+\mathbf{B y}(\bmod$ периодов $)$, $0 \leqslant(\mathbf{x})_{j},(\mathbf{y})_{j}<1, \mathrm{~N}, \mathrm{M} \in \mathbb{Z}^{g} ;$ иногда для краткости знак сравнения по модулю периодов базисных дифференциалов $\left\{d \Omega_{k}\right\}$ мы будем опускать и писать $\mathbf{v} \equiv \mathbf{x}+\mathbf{B y}$.

Неупорядоченные наборы точек $\left(\boldsymbol{\lambda}_{1}, \ldots, \boldsymbol{\lambda}_{g}\right), \boldsymbol{\lambda}_{j} \in \mathfrak{R}$, образуют $g$-ю симметрическую степень $S^{g} \mathfrak{R}$ римановой поверхности $\mathfrak{R}$. Пусть $\boldsymbol{\lambda}_{0}$ - некоторая фиксированная точка ${ }^{7}$ римановой поверхности $\mathfrak{R}$. Вектор-функция $A\left(\boldsymbol{\lambda}_{1}, \ldots, \boldsymbol{\lambda}_{g}\right)=\left(A_{1}, \ldots, A_{g}\right)$ с координатами

$$
A_{k}=A_{k}\left(\boldsymbol{\lambda}_{1}, \ldots, \boldsymbol{\lambda}_{g}\right) \equiv \sum_{j=1}^{g} \Omega_{k}\left(\boldsymbol{\lambda}_{j}\right) \equiv \sum_{j=1}^{g} \int_{\boldsymbol{\lambda}_{0}}^{\boldsymbol{\lambda}_{j}} d \Omega_{k},
$$

где $k=1, \ldots, g$, определяет отображение Абеля $A: S^{g} \mathfrak{R} \rightarrow$ Јас $\mathfrak{R}$ (пути интегрирования в (А.1) - одни и те же для всех $k=1, \ldots, g$ ). Если задан вектор $\mathbf{v}=\left(v_{1}, \ldots, v_{g}\right) \in \mathrm{Jac} \mathfrak{R}$ (точнее, класс $[\mathbf{v}]$ эквивалентных $\mathbf{v}$ векторов), то задача нахождения неупорядоченного набора точек $\left(\boldsymbol{\lambda}_{1}, \ldots, \boldsymbol{\lambda}_{g}\right) \in S^{g} \Re$, для которого

$$
A_{k}\left(\boldsymbol{\lambda}_{1}, \ldots, \boldsymbol{\lambda}_{g}\right)=v_{k} \quad(\bmod \text { периодов }),
$$

где $k=1, \ldots, g$, называется проблемой обращения Якоби. Проблема обращения Якоби всегда разрешима, но, вообще говоря, не единственным образом. Неупорядоченный набора точек $\left(\boldsymbol{\lambda}_{1}, \ldots, \boldsymbol{\lambda}_{g}\right) \in S^{g} \mathfrak{R}$ будем называть дивизором и использовать обозначение $d=\boldsymbol{\lambda}_{1}+\cdots+\boldsymbol{\lambda}_{g}$.

2. Для любых двух различных точек $\boldsymbol{\lambda}_{1}$ и $\boldsymbol{\lambda}_{2}$ римановой поверхности $\mathfrak{R}$ существует абелев дифференциал третьего рода, голоморфный всюду на $\mathfrak{R}$, за исключением этих двух точек, в которых он имеет простые полюсы с вычетами соответственно +1

\footnotetext{
${ }^{7} \mathrm{~B}$ дальнейшем мы выбираем $\boldsymbol{\lambda}_{0}=e_{2 g+2}$.
} 
и - 1 ; такой дифференциал называется нормальным. Нормальный дифференциал будет определен однозначно, если потребовать, чтобы все его $a$-периоды были равны нулю; будем называть такой дифференциал $a$-нормированным абелевым дифференциалом третьего рода и обозначать $d \Omega\left(\boldsymbol{\lambda}_{1}, \boldsymbol{\lambda}_{2} ; \boldsymbol{\lambda}\right)$, где дифференцирование - по переменной $\lambda$. Другой способ однозначно задать (нормировать) дифференциал третьего рода - потребовать, чтобы все его периоды были чисто мнимыми. Для таких дифференциалов будем использовать обозначение $d G\left(\boldsymbol{\lambda}_{1}, \boldsymbol{\lambda}_{2} ; \boldsymbol{\lambda}\right)$, предполагая при этом, что вычеты в точках $\boldsymbol{\lambda}_{1}$ и $\boldsymbol{\lambda}_{2}$ равны соответственно -1 и +1 . К подобным дифференциалам относится дифференциал комплексной функции Грина с особенностью в бесконечно удаленной точке $G(\lambda, \infty)=g(\lambda, \infty)+i g^{*}(\lambda, \infty)$ многосвязной области $D=\widehat{\mathbb{C}} \backslash S$ (здесь $g^{*}(\lambda, \infty)$ - функция, гармонически сопряженная к функции Грина $g(\lambda, \infty))$. Функция $G(\lambda, \infty)$ многозначна в $D$ и

$$
G(\lambda, \infty)=\int_{e_{2 g+2}}^{\lambda} \frac{P_{g}(\zeta)}{\sqrt{h(\zeta)}} d \zeta, \quad \lambda \in D,
$$

где $P_{g}(\lambda)=P_{g}(\lambda ; h)=\lambda^{g}+\cdots-$ вещественный полином, все нули которого лежат в лакунах $\left(e_{2 j}, e_{2 j+1}\right), j=1, \ldots, g$. Дифференциал $d G=d G\left(\infty^{(1)}, \infty^{(2)} ; \boldsymbol{\lambda}\right)=P_{g}(\lambda) d \lambda / w(\boldsymbol{\lambda})$ определен на всей римановой поверхности $\mathfrak{R}$, имеет чисто мнимые периоды и простые полюсы в точках $\boldsymbol{\lambda}=\infty^{(1)}$ и $\boldsymbol{\lambda}=\infty^{(2)}$ с вычетами соответственно -1 и +1 . Тем самым, функция $u(\boldsymbol{\lambda})=\operatorname{Re} G(\boldsymbol{\lambda}, \infty)$, где $G(\boldsymbol{\lambda}, \infty)=\int_{e_{2 g+2}}^{\boldsymbol{\lambda}} d G,-$ однозначная функция на $\mathfrak{R}$ и для $\boldsymbol{\lambda}=\lambda^{(1)}$ имеем: $u\left(\lambda^{(1)}\right)=g(\lambda, \infty)$. Функция $u(\boldsymbol{\lambda})$ задает естественное продолжение функции Грина $g(\lambda, \infty)$ на всю риманову поверхность $\mathfrak{R}: g(\boldsymbol{\lambda}, \infty)=u(\boldsymbol{\lambda})$.

Приведем используемые в дальнейшем "явные" представления для некоторых $a$-нормированных абелевых дифференциалов третьего рода:

$$
\begin{aligned}
d \Omega\left(\lambda_{1}^{(1)}, \lambda_{2}^{(1)} ; \boldsymbol{\lambda}\right)= & \frac{w(\boldsymbol{\lambda})+w\left(\boldsymbol{\lambda}_{1}\right)}{\lambda-\lambda_{1}} \frac{d \lambda}{2 w(\boldsymbol{\lambda})} \\
& -\frac{w(\boldsymbol{\lambda})+w\left(\boldsymbol{\lambda}_{2}\right)}{\lambda-\lambda_{2}} \frac{d \lambda}{2 w(\boldsymbol{\lambda})}+\frac{p_{1}(\lambda)}{2 w(\boldsymbol{\lambda})} d \lambda, \\
d \Omega\left(\lambda_{1}^{(1)}, \infty^{(2)} ; \boldsymbol{\lambda}\right)= & \frac{w(\boldsymbol{\lambda})+w\left(\boldsymbol{\lambda}_{1}\right)}{\lambda-\lambda_{1}} \frac{d \lambda}{2 w(\boldsymbol{\lambda})}-\frac{\lambda^{g}+p_{2}(\lambda)}{2 w(\boldsymbol{\lambda})} d \lambda,
\end{aligned}
$$




$$
d \Omega\left(\infty^{(1)}, \infty^{(2)} ; \boldsymbol{\lambda}\right)=-\frac{\lambda^{g}+p_{3}(\lambda)}{w(\boldsymbol{\lambda})} d \lambda,
$$

где $p_{j} \in \mathbb{C}[\lambda], \operatorname{deg} p_{j} \leqslant g-1$.

3. Как хорошо известно [30], $a$-периоды дифференциала комплексной функции Грина связаны с гармоническими мерами $\omega_{j}(\infty)$ следующим образом:

$$
\oint_{\mathbf{a}_{k}} d G\left(\infty^{(1)}, \infty^{(2)} ; \boldsymbol{\lambda}\right)=-2 \pi i \omega_{k}(\infty) .
$$

Кроме того, из вещественности $\mathfrak{R}$ вытекает, что все

$$
\oint_{\mathbf{b}_{k}} d G\left(\infty^{(1)}, \infty^{(2)} ; \boldsymbol{\lambda}\right)=0 .
$$

В дальнейшем нам понадобится следующее соотношение ([25], формула (26)) между дифференциалом $d G$ и $a$-нормированным дифференциалом $d \Omega$ :

$$
d G+2 \pi i \sum_{j=1}^{g} \omega_{j}(\infty) d \Omega_{j}+d \Omega=0 .
$$

Справедливость (А.3) следует непосредственно из того, что слева в этой формуле стоит голоморфный абелев дифференциал с нулевыми $a$-периодами.

Рассеченная риманова поверхность $\widetilde{\mathfrak{R}}$ получается из $\mathfrak{R}$ проведением сечений по базисным циклам $\mathbf{a}_{k}, \underline{\sim}_{k}, k=1, \ldots, g$, поверхности: $\widetilde{\Re}=\mathfrak{R} \backslash\left(\bigcup_{k=1}^{g} \mathbf{a}_{k} \cup \bigcup_{k=1}^{g} \mathbf{b}_{k}\right) ; \widetilde{\Re}$ связна (переход с одного листа на другой осуществляется через $\left.\boldsymbol{\Gamma}_{g+1}\right)$ и односвязна. Для $b$-периодов $a$-нормированного абелева дифференциала $d \Omega\left(\boldsymbol{\lambda}_{1}, \boldsymbol{\lambda}_{2} ; \boldsymbol{\lambda}\right)$ справедливо следующее соотношение Римана [32]:

$$
\begin{aligned}
\int_{\mathbf{b}_{k}} d \Omega\left(\boldsymbol{\lambda}_{1}, \boldsymbol{\lambda}_{2} ; \boldsymbol{\zeta}\right) & =-2 \pi i \int_{\boldsymbol{\lambda}_{1}}^{\boldsymbol{\lambda}_{2}} d \Omega_{k}(\boldsymbol{\zeta}) \\
& =2 \pi i\left(\Omega_{k}\left(\boldsymbol{\lambda}_{1}\right)-\Omega_{k}\left(\boldsymbol{\lambda}_{2}\right)\right), \quad k=1, \ldots, g
\end{aligned}
$$

где интеграл берется по произвольному пути, соединяющему точки $\boldsymbol{\lambda}_{1}$ и $\boldsymbol{\lambda}_{2}$ и лежащему в $\widetilde{\Re}$. Используя (А.4), вычислим (нулевые) $b$-периоды левой части (А.3). Получим следующее соотношение

$$
\int_{\infty(1)}^{\infty^{(2)}} d \Omega_{k}=\sum_{j=1}^{g} \omega_{j}(\infty) B_{k j}, \quad k=1, \ldots, g .
$$


Откуда вытекает равенство

$$
2 \Omega_{k}\left(\infty^{(2)}\right)=-2 \Omega_{k}\left(\infty^{(1)}\right)=\sum_{j=1}^{g} \omega_{j}(\infty) B_{k j}, \quad k=1, \ldots, g
$$

(напомним, что $\Omega_{k}\left(e_{2 g+2}\right)=0$ в $\widetilde{\mathfrak{R}}$ ).

Формула (А.3) допускает следующее обобщение для $x \in \mathbb{R} \backslash S$

$$
d G\left(x^{(1)}, x^{(2)}, \boldsymbol{\zeta}\right)+2 \pi i \sum_{j=1}^{g} \omega_{j}(x) d \Omega_{j}(\boldsymbol{\zeta})+d \Omega\left(x^{(1)}, x^{(2)}, \boldsymbol{\zeta}\right)=0,
$$

где $d G\left(x^{(1)}, x^{(2)}, \boldsymbol{\zeta}\right)$ - нормальный абелев дифференциал с чисто мнимыми периодами и с двумя простыми полюсами в точках $x^{(1)}$ и $x^{(2)}$, имеющий в них вычеты -1 и +1 соответственно, $d \Omega\left(x^{(1)}, x^{(2)}, \boldsymbol{\zeta}\right)$ - $a$-нормированный абелев дифференциал с двумя простыми полюсами в точках $x^{(1)}$ и $x^{(2)}$, имеющий вычеты в них +1 и -1 соответственно. Функция $u(\boldsymbol{\lambda} ; x):=$ $\operatorname{Re} \int_{e_{2 g+2}}^{\boldsymbol{\lambda}} d G\left(x^{(1)}, x^{(2)}, \boldsymbol{\zeta}\right)$ однозначна на $\mathfrak{R}$ и при $\boldsymbol{\lambda}=\lambda^{(1)}$ справедливо равенство $u(\boldsymbol{\lambda} ; x)=g(\lambda, x)$, где $g(\lambda, x)=g_{D}(\lambda, x)$ - функция Грина области $D$ с особенностью в точке $\lambda=x$. Тем самым, $g(\lambda, x)$ продолжается на всю $\mathfrak{R}$ как однозначная гармоническая функция в $\mathfrak{R} \backslash\left\{x^{(1)}, x^{(2)}\right\}$.

Как известно (см., например, [31], формула (1.5)) справедливо следующее правило перестановки пределов интегрирования с параметрами для $a$-нормированного дифференциала:

$$
\int_{\mathbf{z}_{1}}^{\mathbf{z}_{2}} d \Omega\left(\boldsymbol{\lambda}_{1}, \boldsymbol{\lambda}_{2} ; \boldsymbol{\zeta}\right)=\int_{\boldsymbol{\lambda}_{1}}^{\boldsymbol{\lambda}_{2}} d \Omega\left(\mathbf{z}_{1}, \mathbf{z}_{2} ; \boldsymbol{\zeta}\right) \quad(\bmod 2 \pi i),
$$

где пути интегрирования лежат в $\widetilde{\Re}$.

Наконец, имеет место следующая связь между гармоническими мерами $\omega_{k}(\lambda)$ отрезков $\Delta_{k}, k=1, \ldots, g$, и абелевыми интегралами $\Omega_{j}(\boldsymbol{\lambda}), j=1, \ldots, g$ :

$$
\sum_{k=1}^{g} B_{k j} \omega_{k}(\lambda)=-2 i \operatorname{Im} \Omega_{j}\left(\lambda^{(1)}\right), \quad \lambda \in D,
$$

или, эквивалентно,

$$
\omega_{k}(\lambda)=-2 \operatorname{Re} \sum_{j=1}^{g} B^{k j} \Omega_{j}\left(\lambda^{(1)}\right), \quad \lambda \in D,
$$


где $\left\|B^{k j}\right\|_{k, j=1, \ldots, g}=\mathbf{B}^{-1}$ (cp. [22], формула (26)). Действительно, все периоды абелева интеграла $\Omega_{j}$ в области $D$ - вещественные. Тем самым, правая часть (А.8) - однозначная гармоническая функция в $D$. Так как величины $B_{k j}$ чисто мнимые, то как нетрудно увидеть, эта правая часть принимает на отрезках $\Delta_{k}$, $k=1, \ldots, g$, значения, равные $B_{k j}$, и нулевое значение - на отрезке $\Delta_{g+1}$. Точно также, очевидно, ведет себя и левая часть (А.8).

4. Дивизором $d$ на римановой поверхности $\mathfrak{R}$ будем называть формальный символ $d=\nu_{1} \boldsymbol{\lambda}_{1}+\nu_{2} \boldsymbol{\lambda}_{2}+\cdots+\nu_{m} \boldsymbol{\lambda}_{m}$, где $\boldsymbol{\lambda}_{j} \in \mathfrak{R}$, $\nu_{j} \in \mathbb{Z} ;|d|=\nu_{1}+\cdots+\nu_{g}-$ степень дивизора $d$; если точки $\boldsymbol{\lambda}_{j}$ попарно различны и все $\nu_{j}>0$, то дивизор $d-$ иелый. Произвольной функции $F$, мероморфной на $\mathfrak{R}$ (тем самым, $F \in \mathbb{C}(\lambda, w)$, т.е. $F=r_{1}(\lambda)+r_{2}(\lambda) w(\boldsymbol{\lambda})$, где $r_{1}, r_{2} \in \mathbb{C}(\lambda)$ - рациональные функции переменной $\lambda)$, сопоставим дивизор $(F)$ ее нулей и полюсов: $(F)=\nu_{1} \boldsymbol{\lambda}_{1}+\cdots+\nu_{\ell} \boldsymbol{\lambda}_{\ell}-\mu_{1} \boldsymbol{\zeta}_{1}-\cdots-\mu_{m} \boldsymbol{\zeta}_{m}$, где $\boldsymbol{\lambda}_{j}, \boldsymbol{\zeta}_{j} \in \mathfrak{R}-$ соответственно нули и полюсы $F$, а $\nu_{j}, \mu_{j}>0$ - их кратности; такие дивизоры называются главными. По теореме Абеля дивизор $d=\nu_{1} \boldsymbol{\lambda}_{1}+\cdots+\nu_{\ell} \boldsymbol{\lambda}_{\ell}-\mu_{1} \boldsymbol{\zeta}_{1}-\cdots-\mu_{m} \boldsymbol{\zeta}_{m}-$ главный тогда и только тогда, когда $|d|=0$ и для всех $k=1, \ldots, g$

$$
\sum_{j=1}^{\ell} \nu_{j} \Omega_{k}\left(\boldsymbol{\lambda}_{j}\right)-\sum_{j=1}^{m} \mu_{j} \Omega_{k}\left(\boldsymbol{\zeta}_{j}\right)=0 \quad(\bmod \text { периодов })
$$

(пути интегрирования здесь - одни и те же для всех $k$ ). Два целых дивизора $d_{1}$ и $d_{2}$ эквивалентны, если их разность $d_{1}-d_{2}-$ главный дивизор. Эквивалентные дивизоры и только они являются решением проблемы обращения Якоби для одного и того же вектора $\mathbf{v}$ из Јас $\mathfrak{R}$. Дивизор $d=\nu_{1} \boldsymbol{\lambda}_{1}+\cdots+\nu_{m} \boldsymbol{\lambda}_{m}-$ специ альный, если соответствующая проблема обращения Якоби имеет не единственное решение; тем самым, он имеет (нетривиальные) эквивалентные ему дивизоры.

Известно (см., например, [31] и [33]), что два целых дивизора степени $g, d_{1}=\boldsymbol{\lambda}_{1}+\cdots+\boldsymbol{\lambda}_{g}$ и $d_{2}=\boldsymbol{\zeta}_{1}+\cdots+\boldsymbol{\zeta}_{g}$, заданных на гиперэллиптической римановой поверхности рода $g$, эквивалентны тогда и только тогда, когда $d_{1}=t_{1}^{(1)}+t_{1}^{(2)}+\cdots+t_{2 m}^{(1)}+t_{2 m}^{(2)}+$ $\boldsymbol{\lambda}_{2 m+1}+\cdots+\boldsymbol{\lambda}_{g}, d_{2}=\tau_{1}^{(1)}+\tau_{1}^{(2)}+\cdots+\tau_{2 m}^{(1)}+\tau_{2 m}^{(2)}+\boldsymbol{\lambda}_{2 m+1}+\cdots+\boldsymbol{\lambda}_{g}$, $m \leqslant g / 2$. Тем самым, имеется полное описание специальных дивизоров степени $g$. Непосредственно отсюда вытекает следующее свойство отображения Абеля $A: S^{g} \mathfrak{R} \rightarrow$ Јас $\mathfrak{R}$. Пусть $\mathscr{U} \subset S^{g} \mathfrak{R}$ - множество, устроенное следующим образом: $\mathscr{U}$ представляет 
собой (неупорядоченное) произведение $g$ непересекающихся компактов $\mathscr{U}_{j}$ в $D^{(1)}, \mathscr{U}_{j} \cap \mathscr{U}_{k}=\varnothing$ при $j \neq k$. Тогда $A^{-1}(A(\mathscr{U}))=\mathscr{U}$ и ограничение $A$ на $\mathscr{U}$ - биекция. Сказанное справедливо, в частности, для случая, когда $\mathscr{U}=\mathbf{L}_{1} \times \cdots \times \mathbf{L}_{g}$.

5. Предположим, что на $\mathfrak{R} \backslash \Gamma$ задана (кусочно) мероморфная функция $F(\boldsymbol{\lambda})$, имеющая полюсы только в бесконечно удаленных точках $\boldsymbol{\lambda}=\infty^{(1)}$ и $\boldsymbol{\lambda}=\infty^{(2)}$ каждый порядка $\leqslant g$, предельные "некасательные" значения которой на $\Gamma$ существуют и удовлетворяют условию:

$$
F^{+}(\zeta)-F^{-}(\zeta)=V(\zeta), \quad \zeta \in \Gamma
$$

где функция $V$ голоморфна на $\boldsymbol{\Gamma}$ как функция переменных $\lambda$ и $w$. Тогда с помощью мероморфного абелева дифференциала

$$
d \Omega(\boldsymbol{\zeta} ; \boldsymbol{\lambda})=\frac{w(\boldsymbol{\zeta})+w(\boldsymbol{\lambda})}{\zeta-\lambda} \frac{d \zeta}{2 w(\boldsymbol{\zeta})},
$$

где дифференцирование - по первому аргументу $\boldsymbol{\zeta}$, функция $F(\boldsymbol{\lambda})$ восстанавливается по граничным условиям (А.9) интегралом типа Коши:

$$
F(\boldsymbol{\lambda})=-\frac{1}{2 \pi i} \int_{\boldsymbol{\Gamma}^{+}} V(\boldsymbol{\zeta}) d \Omega(\boldsymbol{\zeta} ; \boldsymbol{\lambda})+p(\lambda), \quad \boldsymbol{\lambda} \notin \boldsymbol{\Gamma},
$$

где $p(\lambda)$ - некоторый многочлен, $\operatorname{deg} p \leqslant g$, а контур $\boldsymbol{\Gamma}^{+}$ориентирован положительно относительно области $D^{(1)}$ : область остается слева при обходе по контуру.

Действительно, легко проверяются следующие свойства дифференциала (А.10):

$$
\frac{1}{2 \pi i} \int_{\boldsymbol{\Gamma}^{+}} P(\zeta) d \Omega(\zeta ; \boldsymbol{\lambda})=\left\{\begin{array}{cc}
-P(\lambda) / 2, & \boldsymbol{\lambda} \in D^{(1)}, \\
P(\lambda) / 2, & \boldsymbol{\lambda} \in D^{(2)}
\end{array}\right.
$$

где $P$ - произвольный полином, $\operatorname{deg} P \leqslant g ;$ дифференциал $d \Omega(\boldsymbol{\zeta} ; \boldsymbol{\lambda})$ имеет полюсы только в точках $\boldsymbol{\lambda}, \infty^{(1)}, \infty^{(2)}$ с вычетами соответственно $1,-1 / 2,-1 / 2$. Если функция $V$ голоморфна на $\boldsymbol{\Gamma}$, то функция

$$
F_{V}(\boldsymbol{\lambda}):=-\frac{1}{2 \pi i} \int_{\boldsymbol{\Gamma}^{+}} V(\boldsymbol{\zeta}) d \Omega(\boldsymbol{\zeta} ; \boldsymbol{\lambda}), \quad \boldsymbol{\lambda} \notin \boldsymbol{\Gamma},
$$

обладает следующими свойствами: 
1) $F_{V}$ голоморфна в $D^{(1)} \backslash\left\{\infty^{(1)}\right\}$ и $D^{(2)} \backslash\left\{\infty^{(2)}\right\}$;

2) $F_{V}(\boldsymbol{\lambda})=O\left(\lambda^{g}\right)$ при $\boldsymbol{\lambda} \rightarrow \infty^{(1)}$ или $\boldsymbol{\lambda} \rightarrow \infty^{(2)}$;

3) справедлива формула Сохоцкого-Племеля

$$
F_{V}^{ \pm}\left(\boldsymbol{\zeta}_{0}\right)= \pm \frac{1}{2} V\left(\boldsymbol{\zeta}_{0}\right)-\frac{1}{2 \pi i} \int_{\boldsymbol{\Gamma}^{+}} V(\boldsymbol{\zeta}) d \Omega\left(\boldsymbol{\zeta} ; \boldsymbol{\zeta}_{0}\right),
$$

где $\boldsymbol{\zeta}_{0} \in \boldsymbol{\Gamma}$, а последний интеграл понимается в смысле главного значения;

4) $F_{V}^{+}\left(\boldsymbol{\zeta}_{0}\right)-F_{V}^{-}\left(\boldsymbol{\zeta}_{0}\right)=V\left(\boldsymbol{\zeta}_{0}\right), \boldsymbol{\zeta}_{0} \in \boldsymbol{\Gamma}$.

Рассмотрим разность $v(\boldsymbol{\lambda})=F(\boldsymbol{\lambda})-F_{V}(\boldsymbol{\lambda})$. В силу (А.9) и свойства 4) эта функция продолжается на всю $\mathfrak{R}$ как мероморфная функция, т.е $v \in \mathbb{C}(\lambda, w)$, а следовательно, имеет вид $v(\boldsymbol{\lambda})=$ $r_{1}(\lambda)+r_{2}(\lambda) w(\boldsymbol{\lambda}), r_{1}, r_{2} \in \mathbb{C}(\lambda)$. Так как $v$ может иметь полюсы порядка $\leqslant g$ и только в точках $\infty^{(1)}$ и $\infty^{(2)}$, то $r_{j}=p_{j} \in \mathbb{C}[z]$. Следовательно, $v(\boldsymbol{\lambda})=p_{1}(\lambda)+p_{2}(\lambda) w(\boldsymbol{\lambda})$. Учитывая, что $|w(\boldsymbol{\lambda})| \sim|\lambda|^{g+1}$ при $\boldsymbol{\lambda} \rightarrow \infty^{(1)}, \infty^{(2)}$, получаем $p_{2} \equiv 0, \operatorname{deg} p_{1} \leqslant g$. Формула (А.11) доказана.

Отметим наконец, что при $\boldsymbol{\zeta}=\boldsymbol{\zeta}^{(1)}, \boldsymbol{\lambda}=\lambda^{(1)}$ или $\boldsymbol{\zeta}=\boldsymbol{\zeta}^{(2)}$, $\boldsymbol{\lambda}=\lambda^{(2)}$ для дифференциала (А.10) имеем

$$
d \Omega\left(\zeta^{(1)} ; \lambda^{(1)}\right)=d \Omega\left(\zeta^{(2)} ; \lambda^{(2)}\right)=\frac{\sqrt{h(\zeta)}+\sqrt{h(\lambda)}}{\zeta-\lambda} \frac{d \zeta}{2 \sqrt{h(\zeta)}} ;
$$

а при $\zeta=\zeta^{(2)}, \boldsymbol{\lambda}=\lambda^{(1)}$

$$
d \Omega\left(\zeta^{(2)} ; \lambda^{(1)}\right)=\frac{\sqrt{h(\zeta)}-\sqrt{h(\lambda)}}{\zeta-\lambda} \frac{d \zeta}{2 \sqrt{h(\zeta)}},
$$

т.е. в этом случае при $\zeta=\lambda$ дифференциал особенности не имеет. Кроме того, под действием операции инволюции $\boldsymbol{\lambda}^{*}=(\lambda, \mp w)$ при $\boldsymbol{\lambda}=(\lambda, \pm w)$ дифференциал (А.10) преобразуется следующим образом: $d \Omega\left(\boldsymbol{\zeta}^{*}, \boldsymbol{\lambda}\right)=d \Omega\left(\boldsymbol{\zeta}, \boldsymbol{\lambda}^{*}\right)$. Следовательно,

$$
d \Omega\left(\zeta^{(1)}, \lambda^{(2)}\right)=d \Omega\left(\zeta^{(2)}, \lambda^{(1)}\right)=\frac{\sqrt{h(\zeta)}-\sqrt{h(\lambda)}}{\zeta-\lambda} \frac{d \zeta}{2 \sqrt{h(\zeta)}} .
$$

\section{Приложение В. Доказательство существования и вывод явных формул для $\Psi$-функции}

Единственность. Для доказательства единственности решения задачи $(\mathscr{R})$ предположим, что существуют два решения этой 
задачи: $\Psi$ с дивизором свободных нулей $d=\boldsymbol{\lambda}_{1}+\cdots+\boldsymbol{\lambda}_{g}$ и $\widetilde{\Psi}$ с дивизором свободных нулей $\widetilde{d}=\widetilde{\boldsymbol{\lambda}}_{1}+\cdots+\widetilde{\boldsymbol{\lambda}}_{g}$. Так как $\boldsymbol{\lambda}_{j}, \widetilde{\boldsymbol{\lambda}}_{j} \in \mathbf{L}_{j}$, то каждый из дивизоров $d$ и $\widetilde{d}-$ неспециальный. Функция $F(\boldsymbol{\lambda}):=\Psi(\boldsymbol{\lambda}) / \widetilde{\Psi}(\boldsymbol{\lambda})$ - однозначная мероморфная функция на всей римановой поверхности $\mathfrak{R}$ с дивизором $(F)=d-\widetilde{d}=$ $\boldsymbol{\lambda}_{1}+\cdots+\boldsymbol{\lambda}_{g}-\widetilde{\boldsymbol{\lambda}}_{1}-\cdots-\widetilde{\boldsymbol{\lambda}}_{g}$. Из приведенного в п. 4 приложения А свойства эквивалентных дивизоров, заданных на гиперэллиптической римановой поверхности, вытекает, что $\boldsymbol{\lambda}_{j}=\widetilde{\boldsymbol{\lambda}}_{j}$, $j=1,2, \ldots, g$, тем самым, $(F)=0$ и $F \equiv$ const.

Существование. Доказательство существования $\Psi$-функции проведем по следующей схеме. Предположив сначала, что все $\lambda_{j} \in\left(e_{2 j}, e_{2 j+1}\right)$, найдем явный вид этой функции в терминах абелевых интегралов на $\mathfrak{R}$. После чего можно непосредственно проверить, что найденная функция является решением задачи $(\mathscr{R})$, а явная формула дает искомое решение задачи и в случае, когда хотя бы одна точка $\lambda_{j}$ совпадает с концом лакуны $e_{2 j}$ или $e_{2 j+1}$. При этом окажется, что точки $\boldsymbol{\lambda}_{1}, \ldots, \boldsymbol{\lambda}_{g}$ удовлетворяют проблеме обращения Якоби (А.2) с правой частью, имеющей специальный вид. Будет показано, такая специальная задача всегда имеет единственное решение притом такое, что все $\lambda_{j} \in\left[e_{2 j}, e_{2 j+1}\right]$.

1. Итак, пусть $\Psi$ - решение задачи $(\mathscr{R})$, причем такое, что все $\lambda_{j} \in\left(e_{2 j}, e_{2 j+1}\right)$. Положим $v(\lambda):=\Psi\left(\lambda^{(1)}\right) \Psi\left(\lambda^{(2)}\right)$. Нетрудно увидеть, что эта функция продолжается на всю $\widehat{\mathbb{C}}$ как (однозначная) мероморфная функция с полюсом порядка $g$ в точке $\lambda=\infty$ и нулях в точках $\lambda_{1}, \ldots, \lambda_{g}$. Следовательно, $v(\lambda)$ - полином степени $g$, точнее $v(\lambda) \equiv$ const $\prod_{j=1}^{g}\left(\lambda-\lambda_{g}\right)$, где const $\neq 0$. Для последующих рассуждений удобно считать, что const $=1$, тем самым,

$$
\Psi\left(\lambda^{(1)}\right) \Psi\left(\lambda^{(2)}\right) \equiv \prod_{j=1}^{g}\left(\lambda-\lambda_{g}\right) .
$$

Этим соотношением $\Psi$-функция определена однозначно с точностью до знака \pm . В дальнейшем мы уточним выбор знака. Отметим, что фактически $X_{g}(\lambda)=\prod_{j=1}^{g}\left(\lambda-\lambda_{j}\right)$ является неизвестным "полиномиальным параметром" задачи. Для функции $\Psi(\lambda)=\Psi\left(\lambda^{(1)}\right)$ на $S=\bigsqcup_{j=1}^{g+1} \Delta_{j}$ выполняется следующее краевое 
условие $^{8}$ :

$$
\rho(x) \Psi^{+}(x) \Psi^{-}(x)=\prod_{j=1}^{g}\left(x-\lambda_{j}\right), \quad x \in S .
$$

Действительно, пусть $\lambda \rightarrow x \in \Delta_{j}, j \in\{1, \ldots, g+1\}$, причем, $\operatorname{Im} \lambda>0$. Тогда $\Psi\left(\lambda^{(1)}\right) \rightarrow \Psi^{+}(x)$ и, в силу краевого условия $\rho(x) \Psi^{+}(\mathbf{x})=\Psi^{-}(\mathbf{x}), \mathbf{x} \in \boldsymbol{\Gamma}$, имеем: $\Psi\left(\lambda^{(2)}\right) \rightarrow \rho(x) \Psi^{-}(x)$. Теперь (В.2) вытекает непосредственно из (В.1).

2. Рассмотрим $a$-нормированные абелевы дифференциалы третьего рода $d \Omega\left(\infty^{(1)}, \boldsymbol{\lambda}_{j} ; \boldsymbol{\lambda}\right), j=1, \ldots, g$, и $d \Omega\left(\infty^{(1)}, \infty^{(2)} ; \boldsymbol{\lambda}\right)$. Непосредственно из явного представления (см. приложение $\mathrm{A}$, п. 2) для таких абелевых дифференциалов вытекает, что

$$
\begin{aligned}
d \Omega\left(\infty^{(1)}, \boldsymbol{\lambda}_{j} ; \boldsymbol{\lambda}\right)=- & \frac{w(\boldsymbol{\lambda})+w\left(\boldsymbol{\lambda}_{j}\right)}{\lambda-\lambda_{j}} \frac{d \lambda}{2 w(\boldsymbol{\lambda})} \\
& -\left(\lambda^{g}+p(\lambda)\right) \frac{d \lambda}{2 w(\boldsymbol{\lambda})}, \\
d \Omega\left(\infty^{(1)}, \infty^{(2)} ; \boldsymbol{\lambda}\right)= & -\left(\lambda^{g}+q(\lambda)\right) \frac{d \lambda}{2 w(\boldsymbol{\lambda})},
\end{aligned}
$$

где $p, q-$ полиномы степени $\leqslant g-1$. На $\mathfrak{R} \backslash \boldsymbol{\Gamma}$ дифференциалы (В.3) имеют периоды, кратные $2 \pi i$. Следовательно, абелевы интегралы

$$
\begin{aligned}
\Omega\left(\infty^{(1)}, \boldsymbol{\lambda}_{j} ; \boldsymbol{\lambda}\right) & =\int_{e_{2 g+2}}^{\boldsymbol{\lambda}} d \Omega\left(\infty^{(1)}, \boldsymbol{\lambda}_{j} ; \boldsymbol{\zeta}\right), \quad j=1, \ldots, g, \\
\Omega\left(\infty^{(1)}, \infty^{(2)} ; \boldsymbol{\lambda}\right) & =\int_{e_{2 g+2}}^{\boldsymbol{\lambda}} d \Omega\left(\infty^{(1)}, \infty^{(2)} ; \boldsymbol{\zeta}\right),
\end{aligned}
$$

где интегрирование ведется по кривым, целиком лежащим или в $D^{(1)}$, или в $D^{(2)}$, однозначны по модулю $2 \pi i$ на $\mathfrak{R} \backslash \boldsymbol{\Gamma}$. Тем самым функции

$$
\begin{aligned}
& F_{1}(\boldsymbol{\lambda})=\exp \left\{\Omega\left(\infty^{(1)}, \infty^{(2)} ; \boldsymbol{\lambda}\right)\right\} \\
& F_{2}(\boldsymbol{\lambda})=\exp \left\{\sum_{j=1}^{g} \Omega\left(\infty^{(1)}, \boldsymbol{\lambda}_{j} ; \boldsymbol{\lambda}\right)\right\}
\end{aligned}
$$

\footnotetext{
${ }^{8} \mathrm{~B}$ классическом случае $g=0$ непосредственно из этого краевого условия уже вытекает нужное представление функций $\Psi$.
} 
- однозначные (кусочно) мероморфные функции при $\boldsymbol{\lambda} \in \mathfrak{R} \backslash \boldsymbol{\Gamma}$, для дивизоров которых имеем: $\left(F_{1}\right)=\infty^{(1)}-\infty^{(2)},\left(F_{2}\right)=g \infty^{(1)}-$ $\boldsymbol{\lambda}_{1}-\cdots-\boldsymbol{\lambda}_{g}$; кроме того, $F_{1}\left(e_{2 g+2}\right)=F_{2}\left(e_{2 g+2}\right)=1$. Таким образом, функция $F_{3}(\boldsymbol{\lambda})=F_{1}^{n-g}(\boldsymbol{\lambda}) F_{2}(\boldsymbol{\lambda})$ мероморфна в $\mathfrak{R} \backslash \boldsymbol{\Gamma}$ и дивизор $\left(F_{3}\right)=-(\Psi)$. Следовательно, функция $F(\boldsymbol{\lambda})=\Psi(\boldsymbol{\lambda}) F_{3}(\boldsymbol{\lambda})=$ $\Psi(\boldsymbol{\lambda}) F_{1}^{n-g}(\boldsymbol{\lambda}) F_{2}(\boldsymbol{\lambda})$ голоморфна и не имеет нулей в $\mathfrak{R} \backslash \boldsymbol{\Gamma}$.

Предположим теперь, что $\boldsymbol{\lambda}=\lambda^{(1)}$, и найдем явное представление функции $\Psi\left(\lambda^{(1)}\right)$.

Непосредственно из представлений (В.3) вытекает, что при $x \in S$ выполняются следующие краевые условия:

$$
\begin{aligned}
d \Omega^{+}\left(\infty^{(1)}, \infty^{(2)} ; x\right)+d \Omega^{-}\left(\infty^{(1)}, \infty^{(2)} ; x\right) & =0, \\
d \Omega^{+}\left(\infty^{(1)}, \boldsymbol{\lambda}_{j} ; x\right)+d \Omega^{-}\left(\infty^{(1)}, \boldsymbol{\lambda}_{j} ; x\right) & =-\frac{d x}{x-\lambda_{j}} .
\end{aligned}
$$

Тем самым, для функций $F_{1}$ и $F_{2}$ имеем:

$$
\begin{aligned}
& F_{1}^{+}(x) F_{1}^{-}(x)=C_{1, k}, \\
& F_{2}^{+}(x) F_{2}^{-}(x)=\frac{C_{2, k}}{\prod_{j=1}^{g}\left(x-\lambda_{j}\right)}, \quad x \in \Delta_{k}, \quad k=1, \ldots, g+1,
\end{aligned}
$$

где постоянные равны: $C_{1, g+1}=1$,

$$
\begin{aligned}
& C_{1, k}=\exp \left\{-\int_{\mathbf{b}_{k}} d \Omega\left(\infty^{(1)}, \infty^{(2)} ; \boldsymbol{\zeta}\right)\right\}, \\
& C_{2, k}=C_{2, g+1} \cdot \exp \left\{-\int_{\mathbf{b}_{k}} \sum_{j=1}^{g} d \Omega\left(\infty^{(1)}, \boldsymbol{\lambda}_{j} ; \boldsymbol{\zeta}\right)\right\}, \quad k=1, \ldots, g,
\end{aligned}
$$

a $C_{2, g+1}=\prod_{j=1}^{g}\left(e_{2 g+2}-\lambda_{j}\right)$. Отсюда вытекают краевые условия для функции $F_{3}=F_{1}^{n-g} F_{2}$ :

$$
F_{3}^{+}(x) F_{3}^{-}(x)=\frac{C_{k}}{\prod_{j=1}^{g}\left(x-\lambda_{j}\right)}, \quad x \in \Delta_{k}, \quad k=1, \ldots, g+1,
$$

где постоянные

$$
\begin{aligned}
C_{k}=C_{g+1} & \exp \left\{-(n-g) \int_{\mathbf{b}_{k}} d \Omega\left(\infty^{(1)}, \infty^{(2)} ; \boldsymbol{\zeta}\right)\right\} \\
& \times \exp \left\{-\int_{\mathbf{b}_{k}} \sum_{j=1}^{g} d \Omega\left(\infty^{(1)}, \boldsymbol{\lambda}_{j} ; \boldsymbol{\zeta}\right)\right\}, \quad k=1, \ldots, g,
\end{aligned}
$$


a $C_{g+1}=\prod_{j=1}^{g}\left(e_{2 g+2}-\lambda_{j}\right)$.

3. Воспользуемся теперь граничным условием $\rho(\zeta) \Psi^{+}(\mathbf{x})=$ $\Psi^{-}(\mathbf{x}), \mathbf{x} \in \boldsymbol{\Gamma}$, и равенством $\Psi\left(\lambda^{(1)}\right) \Psi\left(\lambda^{(2)}\right)=\prod_{j=1}^{g}\left(\lambda-\lambda_{j}\right)$ при $\lambda \in D$. Из этих соотношений получаем $\rho(x) \Psi^{+}(x) \Psi^{-}(x)=\prod_{j=1}^{g}\left(x-\lambda_{j}\right)$, $x \in S$.

Положим $f_{1}(\lambda):=F\left(\lambda^{(1)}\right)=\Psi\left(\lambda^{(1)}\right) F_{3}\left(\lambda^{(1)}\right)$. Тогда функция $f_{1}(\lambda)$ голоморфна и отлична от нуля в области $D$ и для нее выполняются следующие краевые условия:

$$
\begin{aligned}
& f_{1}^{+}(x) f_{1}^{-}(x)= \Psi^{+}(x) \Psi^{-}(x) F_{3}^{+}(x) F_{3}^{-}(x) \\
&= \frac{\prod_{j=1}^{g}\left(x-\lambda_{j}\right)}{\rho(x)} \frac{C_{k}}{\prod_{j=1}^{g}\left(x-\lambda_{j}\right)}=\frac{C_{k}}{\rho(x)}, \\
& x \in \Delta_{k}, \quad k=1, \ldots, g+1,
\end{aligned}
$$

где постоянные $C_{k}$ заданы соотношениями (В.5). Функция $u_{1}(\lambda)=\log f_{1}(\lambda)$ определена при всех $\lambda \in D$, но вообе говоря многозначна в $D$; ее многозначность обусловлена только многосвязностью $D$, а периоды - целые кратные $2 \pi i$. Ясно, что при надлежащем выборе целых чисел $m_{j}, j=1, \ldots, g$, функция $2 \pi i \sum_{j=1}^{g} m_{j} \Omega_{j}(\lambda)$ имеет те же периоды, что и функция $u_{1}$. Тем самым, для функции

$$
u_{2}(\lambda)=u_{1}(\lambda)-2 \pi i \sum_{j=1}^{g} m_{j} \Omega_{j}(\lambda)
$$

имеем:

(i) $u_{2}(\lambda)$ - голоморфная функция в $D$;

(ii) на $S$ выполняются краевые условия:

$$
\begin{gathered}
u_{2}^{+}(x)+u_{2}^{-}(x)=-\log \rho(x)+c_{g+1}+2 \pi i n_{g+1}, \quad x \in \Delta_{g+1}, \\
u_{2}^{+}(x)+u_{2}^{-}(x)=-\log \rho(x)+c_{k}+2 \pi i n_{k}+2 \pi i \sum_{j=1}^{g} m_{j} B_{k j}, \\
x \in \Delta_{k}, \quad k=1, \ldots, g,
\end{gathered}
$$


где $\left(\right.$ см. (B.5)), $c_{g+1}=\log C_{g+1}$,

$$
\begin{aligned}
c_{k} & =\log C_{k}=c_{g+1}+\left\{-(n-g) \int_{\mathbf{b}_{k}} d \Omega\left(\infty^{(1)}, \infty^{(2)} ; \boldsymbol{\zeta}\right)\right\} \\
& +\left\{-\int_{\mathbf{b}_{k}} \sum_{j=1}^{g} d \Omega\left(\infty^{(1)}, \boldsymbol{\lambda}_{j} ; \boldsymbol{\zeta}\right)\right\}, \quad k=1, \ldots, g
\end{aligned}
$$

в дальнейшем (см. ниже п. 5) будет показано, что при условии $\lambda_{j} \in\left[e_{2 j}, e_{2 j+1}\right]$ все $n_{k}=n_{g+1}, k=1, \ldots, g$. В таком случае для функции $u_{3}(\lambda)=u_{2}(\lambda) / w(\lambda)$, голоморфной в $D$ и имеющей в точке $\lambda=\infty$ нуль кратности $g+1$, выполняются следующие краевые условия

$$
\begin{aligned}
u_{3}^{+}(x)-u_{3}^{-}(x) & =-\frac{\log \rho(x)}{w^{+}(x)}+\frac{c_{g+1}}{w^{+}(x)}+\frac{2 \pi i n_{g+1}}{w^{+}(x)}, \\
x \in \Delta_{g+1} & \\
u_{3}^{+}(x)-u_{3}^{-}(x) & =-\frac{\log \rho(x)}{w^{+}(x)}+\frac{c_{k}}{w^{+}(x)}+\frac{2 \pi i n_{k}}{w^{+}(x)} \\
+2 \pi i & \sum_{j=1}^{g} \frac{m_{j} B_{k j}}{w^{+}(x)}, \quad x \in \Delta_{k}, \quad k=1, \ldots, g,
\end{aligned}
$$

Функция $u_{3}(\lambda)$ восстанавливается в области $D$ по этим краевым условиям интегралом типа Коши, а нужная кратность нуля этого интеграла в бесконечно удаленной точке достигается при выполнении $g$ условий ортогональности:

$$
\begin{aligned}
& -\frac{1}{\pi i} \int_{S} \log \rho(x) \frac{x^{\ell-1}}{w^{+}(x)} d x \\
& \quad+\sum_{k=1}^{g+1}\left(c_{k} \frac{1}{\pi i} \int_{\Delta_{k}} \frac{x^{\ell-1}}{w^{+}(x)} d x+2 n_{k} \int_{\Delta_{k}} \frac{x^{\ell-1}}{w^{+}(x)} d x\right) \\
& \quad+\sum_{k=1}^{g} \sum_{j=1}^{g} 2 m_{j} B_{k j} \int_{\Delta_{k}} \frac{x^{\ell-1}}{w^{+}(x)} d x=0, \quad \ell=1, \ldots, g .
\end{aligned}
$$

Заменяя в левой части последней формулы дифференциалы $x^{\ell-1} d \zeta / w(x)$ на нормированные дифференциалы $d \Omega_{\ell}$ и пользуясь тем, что

$$
\int_{\Delta_{k}} d \Omega_{\ell}^{+}=\frac{1}{2} \delta_{k \ell}, \quad \int_{\Delta_{g+1}} d \Omega_{\ell}^{+}=-\frac{1}{2}, \quad k, \ell=1, \ldots, g,
$$


получаем

$$
\begin{aligned}
& \frac{1}{\pi i} \int_{S} \log \rho(x) d \Omega_{\ell}^{+}(x) \\
& \quad=\frac{1}{2 \pi i}\left(c_{\ell}-c_{g+1}\right)+\left(n_{\ell}-n_{g+1}\right)+\sum_{j=1}^{g} m_{j} B_{\ell j} \\
& \quad=\frac{1}{2 \pi i}\left(c_{\ell}-c_{g+1}\right) \quad(\bmod \text { периодов }), \ell=1, \ldots, g .
\end{aligned}
$$

Воспользуемся теперь в очередной раз соотношением Римана (А.4) и преобразуем выражение (В.6) к виду:

$$
c_{\ell}=c_{g+1}+2 \pi i\left\{\sum_{j=1}^{g} \int_{\infty^{(1)}}^{\boldsymbol{\lambda}_{j}} d \Omega_{\ell}(\boldsymbol{\zeta})+(n-g) \int_{\infty^{(1)}}^{\infty^{(2)}} d \Omega_{\ell}(\boldsymbol{\zeta})\right\} .
$$

Наконец, из (В.8) и (В.9) получаем

$$
\begin{aligned}
\sum_{j=1}^{g} \int_{\infty(1)}^{\boldsymbol{\lambda}_{j}} d \Omega_{\ell}(\boldsymbol{\zeta}) & \\
= & \frac{1}{\pi i} \int_{S} \log \rho(\zeta) d \Omega_{\ell}^{+}(\zeta)-(n-g) \int_{\infty^{(1)}}^{\infty^{(2)}} d \Omega_{\ell}(\boldsymbol{\zeta}) \\
& -\left(n_{\ell}-n_{g+1}\right)-\sum_{j=1}^{g} m_{j} B_{\ell j} \\
\equiv & \frac{1}{\pi i} \int_{S} \log \rho(\zeta) d \Omega_{\ell}^{+}(\zeta) \\
& -(n-g) \int_{\infty}^{\infty^{(1)}} d \Omega_{\ell}(\zeta), \quad \ell=1, \ldots, g .
\end{aligned}
$$

Займемся теперь преобразованием к нужному виду последнего интеграла в выражении (В.10).

4. Хорошо известна $([30, \S 4,(4.3)])$ следующая формула для $a$-периодов дифференциала комплексной функции Грина: $\oint_{\mathbf{a}_{k}} d G(\boldsymbol{\zeta})=-2 \pi i \omega_{k}(\infty)$, где $\omega_{k}(\infty), k=1, \ldots, g,-$ гармоническая мера $k$-го отрезка $\Delta_{k}$ относительно области $D$ в бесконечно удаленной точке, $\omega_{k}(\infty)>0, \sum_{k=1}^{g+1} \omega_{k}(\infty)=1$. Непосредственно из 
этой формулы для $a$-периодов дифференциала $d G(\boldsymbol{\zeta})$ вытекает, что справедливо следующее тождество:

$$
d G(\boldsymbol{\zeta})+2 \pi i \sum_{k=1}^{g} \omega_{k}(\infty) d \Omega_{k}(\boldsymbol{\zeta})+d \Omega\left(\infty^{(1)}, \infty^{(2)} ; \boldsymbol{\zeta}\right)=0 .
$$

Действительно, слева в последней формуле стоит голоморфный абелев дифференциал, все $a$-периоды которого равны нулю; следовательно, этот дифференциал равен нулю тождественно. Вычисляя теперь (нулевые) b- периоды этого дифференциала и пользуясь соотношением Римана (А.4), получим (напомним, что интегрирование ведется по путям, лежащим в рассеченной римановой поверхности)

$$
\int_{\infty(1)}^{\infty^{(2)}} d \Omega_{j}(\boldsymbol{\zeta})=\frac{1}{2 \pi i} \oint_{\mathbf{b}_{j}} d G(\boldsymbol{\zeta})+\sum_{k=1}^{g} \omega_{k}(\infty) B_{k j}, \quad j=1, \ldots, g,
$$

где в силу того, что все $e_{j} \in \mathbb{R}$, имеем $\oint_{\mathbf{b}_{j}} d G(\boldsymbol{\zeta})=0$. Тем самым,

$$
\int_{\infty(1)}^{\infty^{(2)}} d \Omega_{j}(\zeta)=\sum_{k=1}^{g} \omega_{k}(\infty) B_{k j}, \quad j=1, \ldots, g .
$$

Следовательно, $x$-координаты вектора $v_{j}=\int_{\infty^{(1)}}^{\infty^{(2)}} d \Omega_{j}(\boldsymbol{\zeta}), j=$ $1, \ldots, g$, в многообразии Якоби Јас $\Re$ равны 0 , а $y$-координаты равны $\omega_{j}(\infty) \in(0,1), j=1, \ldots, g$. Соответственно, вектор с компонентами $v_{j}(n)=(n-g) \int_{\infty(1)}^{\infty^{(2)}} d \Omega_{j}(\boldsymbol{\zeta})$ имеет нулевые $x$ координаты и $y$-координаты, равные $\left\{(n-g) \omega_{j}(\infty)\right\}$; здесь и ниже $\{\cdot\}$ обозначает дробную часть числа. Из (В.10) вытекает, что

$$
\begin{gathered}
\sum_{j=1}^{g} \int_{\infty(1)}^{\boldsymbol{\lambda}_{j}} d \Omega_{\ell}(\boldsymbol{\zeta})=\frac{i}{\pi} \int_{S} \log \rho(x) d \Omega_{\ell}^{+}(x)-\sum_{j=1}^{g}(n-g) \omega_{j}(\infty) B_{\ell j} \\
\quad-\left(n_{\ell}-n_{g+1}\right)-\sum_{j=1}^{g} m_{j} B_{\ell j} \\
=\frac{i}{\pi} \int_{S} \log \rho(x) d \Omega_{\ell}^{+}(x)-\sum_{j=1}^{g} \theta_{j}(n) B_{\ell j}-\left(n_{\ell}-n_{g+1}\right) \equiv
\end{gathered}
$$




$$
\begin{aligned}
& \equiv \frac{i}{\pi} \int_{S} \log \rho(\zeta) d \Omega_{\ell}^{+}(\zeta) \\
& \quad-\sum_{j=1}^{g}\left\{(n-g) \omega_{j}(\infty)\right\} B_{\ell j}, \quad \ell=1, \ldots, g,
\end{aligned}
$$

где $\theta_{k}=\theta_{k}(n)=\ell_{k}(n)+\left\{(n-g) \omega_{k}(\infty)\right\}$, причем целые числа $\ell_{k}(n) \in \mathbb{Z}$ равномерно ограничены при $n \rightarrow \infty$. Последнее свойство вытекает из того, что левая часть (В.12) равномерно ограничена при $n \rightarrow \infty$, так как $\lambda_{j} \in\left[e_{2 j}, e_{2 j+1}\right]$, а $n_{\ell}=n_{g+1}, \ell=1, \ldots, g$. Из (В.12) в силу (А.5) и соотношения

$$
\int_{\infty(1)}^{e_{2 g+2}} d \Omega_{\ell}=\frac{1}{2} \sum_{j=1}^{g} \omega_{j}(\infty) B_{j \ell}
$$

получаем

$$
\begin{aligned}
\sum_{j=1}^{g} \Omega_{k}\left(\boldsymbol{\lambda}_{j}\right)= & \frac{i}{\pi} \int_{S} \log \rho(x) d \Omega_{\ell}^{+}(x)-\sum_{j=1}^{g}\left(n-g+\frac{1}{2}\right) \omega_{j}(\infty) B_{\ell j} \\
& -\left(n_{\ell}-n_{g+1}\right)-\sum_{j=1}^{g} m_{j} B_{\ell j} \\
\equiv & \frac{i}{\pi} \int_{S} \log \rho(\zeta) d \Omega_{\ell}^{+}(\zeta)-\sum_{j=1}^{g}\left\{\left(n-g+\frac{1}{2}\right) \omega_{k}(\infty)\right\} B_{k j},
\end{aligned}
$$

(напомним, что начальная точка интегрирования дифференциалов равна $\left.e_{2 g+2}\right)$ Это и есть та проблема обращения Якоби, которую мы имели ввиду в п. $3 \S 2$ (ср. с [35]). Доказательство существования при каждом $n$ решения этой задачи при условии, что функция $\widehat{\mu}-$ марковская, т.е. $i \rho(x) / w^{+}(x)=\rho(x) / \sqrt{-h(x)}>0$ на $S$, а решение ищется такое, что все $\lambda_{j} \in\left[e_{2 j}, e_{2 j+1}\right]$, будет дано ниже в п. 5; при этом будет показано, что все $n_{\ell}=n_{g+1}$, $\ell=1, \ldots, g$. Предположим пока, что при $n \in \mathbb{N}$ существует решение задачи (В.12), притом такое, что все $\lambda_{j}$ лежат внутри соответствующих лакун. Продолжим при этом условии вывод явной формулы для функции $\Psi\left(\lambda^{(1)}\right)$.

Запишем формулы (В.7) для скачка функции $u_{3}(x)$ в следующем виде

$$
u_{3}^{+}(x)-u_{3}^{-}(x)=-\frac{\log \rho(x)}{w^{+}(x)}+\frac{c_{g+1}}{w^{+}(x)}+\frac{2 \pi i n_{g+1}}{w^{+}(x)}, \quad x \in \Delta_{g+1},
$$




$$
\begin{gathered}
u_{3}^{+}(x)-u_{3}^{-}(x)=-\frac{\log \rho(x)}{w^{+}(x)}+\frac{c_{g+1}}{w^{+}(x)}+\frac{2 \pi i n_{g+1}}{w^{+}(x)}+\frac{c_{k}-c_{g+1}}{w^{+}(x)} \\
+\frac{2 \pi i\left(n_{k}-n_{g+1}\right)}{w^{+}(x)}+\sum_{j=1}^{g} \frac{2 \pi i m_{j} B_{k j}}{w^{+}(x)} \\
x \in \Delta_{k}, k=1, \ldots, g .
\end{gathered}
$$

Первая часть (В.8) - это точное равенство, поэтому последние краевые условия приводятся к виду

$$
\begin{gathered}
u_{3}^{+}(x)-u_{3}^{-}(x)=-\frac{\log \rho(x)}{w^{+}(x)}+\frac{c_{g+1}}{w^{+}(x)}+\frac{2 \pi i n_{g+1}}{w^{+}(x)}, x \in \Delta_{g+1}, \\
u_{3}^{+}(x)-u_{3}^{-}(x)=-\frac{\log \rho(x)}{w^{+}(x)}+\frac{c_{g+1}}{w^{+}(x)}+\frac{2 \pi i n_{g+1}}{w^{+}(x)}+\frac{v_{k}}{w^{+}(x)}, \\
x \in \Delta_{k}, k=1, \ldots, g,
\end{gathered}
$$

где $v_{k}=2 \int_{S} \log \rho(t) d \Omega_{k}^{+}(t)$. Непосредственно из (В.13) вытекает следующее представление для функции $u_{3}$ :

$$
u_{3}(\lambda)=\frac{1}{2 \pi i} \int_{S} \frac{\log \rho(x) d x}{(x-\lambda) w^{+}(x)}+\frac{c_{g+1}}{2 w(\lambda)}+\frac{\pi i n_{g+1}}{w(\lambda)}+v(\lambda),
$$

где

$$
v(\lambda)=\sum_{j=1}^{g} \frac{v_{j}}{2 \pi i} \int_{\Delta_{j}} \frac{d x}{(\lambda-x) w^{+}(x)},
$$

a $e^{c_{g+1}}=C_{g+1}=\prod_{j=1}^{g}\left(e_{2 g+2}-\lambda_{j}\right)$. Отсюда получаем

$$
\begin{aligned}
u_{2}(\lambda) & =w(\lambda) u_{3}(\lambda) \\
& =\frac{w(\lambda)}{2 \pi i} \int_{S} \frac{\log \rho(x) d x}{(x-\lambda) w^{+}(x)}+\frac{c_{g+1}}{2}+\pi i n_{g+1}+v(\lambda) w(\lambda) .
\end{aligned}
$$

Так как $u_{1}(\lambda)=u_{2}(\lambda)+2 \pi i \sum_{j=1}^{g} m_{j} \Omega_{j}(\lambda)$, то для функции $f_{1}(\lambda)$ имеем:

$$
\begin{aligned}
f_{1}(\lambda) & =e^{u_{1}(\lambda)}=\exp \left\{\frac{w(\lambda)}{2 \pi i} \int_{S} \frac{\log \rho(x) d x}{(x-\lambda) w^{+}(x)}+\frac{c_{g+1}}{2}+\pi i n_{g+1}\right. \\
& \left.+v(\lambda) w(\lambda)+2 \pi i \sum_{j=1}^{g} m_{j} \Omega_{j}(\lambda)\right\},
\end{aligned}
$$


где $e^{c_{g+1} / 2}=\sqrt{C_{g+1}}=\sqrt{\prod_{j=1}^{g}\left(e_{2 g+2}-\lambda_{j}\right)}>0$. Нормировкой $\Psi\left(\lambda^{(1)}\right) \Psi\left(\lambda^{(2)}\right)=\prod_{j=1}^{g}\left(\lambda-\lambda_{j}\right)$ функция $\Psi$ определяется однозначно с точностью до сомножителя \pm 1 , этому факту соответствует величина $\exp \left\{\pi i n_{g+1}\right\}$ в представлении (В.14). Тем самым, в дальнейшем мы можем считать, что $n_{g+1}=0$.

Проинтегрируем теперь (тождественно нулевой) дифференциал, стоящий в левой части формулы (В.11), по пути, ведущему от точки $e_{2 g+2}$ до произвольной точки $\boldsymbol{\lambda} \in \mathfrak{R}$ и лежащему в рассеченной римановой поверхности $\widetilde{\Re}$. Получим следующее равенство

$$
\Omega\left(\infty^{(1)}, \infty^{(2)} ; \boldsymbol{\lambda}\right)=-G(\boldsymbol{\lambda}, \infty)-2 \pi i \sum_{j=1}^{g} \omega_{j}(\infty) \Omega_{j}(\boldsymbol{\lambda}) .
$$

Напомним, что $f_{1}(\lambda)=F\left(\lambda^{(1)}\right)=\Psi\left(\lambda^{(1)}\right) F_{3}\left(\lambda^{(1)}\right)$, где $F_{3}=$ $F_{1}^{n-g} F_{2}$, а функции $F_{1}$ и $F_{2}$ заданы (B.4). Учитывая (B.4) и (В.15), получаем из (В.14) следующее представление для функции $\Psi\left(\lambda^{(1)}\right)$, справедливое при условии, что все $\lambda_{j} \in\left(e_{2 j}, e_{2 j+1}\right)$ :

$$
\begin{aligned}
\Psi\left(\lambda^{(1)}\right)= & e^{(n-g) G(\lambda, \infty)} \exp \left\{-\sum_{j=1}^{g} \Omega\left(\infty^{(1)}, \boldsymbol{\lambda}_{j} ; \lambda^{(1)}\right)\right\} \\
& \times \exp \left\{\frac{w(\lambda)}{2 \pi i} \int_{S} \frac{\log \rho(x) d x}{(x-\lambda) w^{+}(x)}+\frac{c_{g+1}}{2}+v(\lambda) w(\lambda)\right\} \\
& \times \exp \left\{2 \pi i \sum_{j=1}^{g}\left((n-g) \omega_{j}(\infty)+m_{j}\right) \Omega_{j}\left(\lambda^{(1)}\right)\right\} \\
= & \Phi^{n-g}(\lambda) \exp \left\{\sum_{j=1}^{g} \Omega\left(\boldsymbol{\lambda}_{j}, \infty^{(1)} ; \lambda^{(1)}\right)\right\} \times \\
& \times \exp \left\{\frac{w(\lambda)}{2 \pi i} \int_{S} \frac{\log \rho(x) d x}{(x-\lambda) w^{+}(x)}+\frac{c_{g+1}}{2}+v(\lambda) w(\lambda)\right\} \\
& \times \exp \left\{2 \pi i \sum_{j=1}^{g} \theta_{j}(n) \Omega_{j}\left(\lambda^{(1)}\right)\right\},
\end{aligned}
$$

где $\Phi(\lambda)=e^{G(\lambda, \infty)}-$ (многозначная) отображающая функция, где $\theta_{k}=\theta_{k}(n)=\ell_{k}(n)+\left\{(n-g) \omega_{k}(\infty)\right\}$, целые числа $\ell_{k}(n) \in \mathbb{Z}$ равномерно ограничены при $n \rightarrow \infty$. 
Положим теперь $f_{2}(\lambda)=F\left(\lambda^{(2)}\right)=\Psi\left(\lambda^{(2)}\right) F_{3}\left(\lambda^{(2)}\right)$. Нетрудно видеть, что для функции $\Psi_{2}(\lambda)=\Psi\left(\lambda^{(2)}\right), \lambda \in D$, выполняется краевое условие: $\Psi_{2}^{+}(x) \Psi_{2}^{-}(x)=\rho(x) X_{g}(x), x \in S$ (ср. с (В.2)). Используя это краевое условие нетрудно доказать, что для функции $f_{2}$, голоморфной и отличной от нуля в области $D$, выполняется равенство $f_{2}(\lambda)=1 / f_{1}(\lambda), \lambda \in D$. Отсюда уже легко вытекает следующее представление для $\Psi$-функции при $\boldsymbol{\lambda}=\lambda^{(2)}$ :

$$
\begin{aligned}
\Psi\left(\lambda^{(2)}\right)= & e^{(g-n) G(\lambda, \infty)} \exp \left\{-\sum_{j=1}^{g} \Omega\left(\infty^{(1)}, \boldsymbol{\lambda}_{j} ; \lambda^{(2)}\right)\right\} \\
& \times \exp \left\{\frac{w(\lambda)}{2 \pi i} \int_{S} \frac{\log \rho(x) d x}{(\lambda-x) w^{+}(x)}-\frac{c_{g+1}}{2}-v(\lambda) w(\lambda)\right\} \\
& \times \exp \left\{2 \pi i \sum_{j=1}^{g}\left((n-g) \omega_{j}(\infty)+m_{j}\right) \Omega_{j}\left(\lambda^{(2)}\right)\right\} \\
= & \frac{1}{\Phi^{n-g}(\lambda)} \exp \left\{\sum_{j=1}^{g} \Omega\left(\boldsymbol{\lambda}_{j}, \infty^{(1)} ; \lambda^{(2)}\right)\right\} \\
& \times \exp \left\{\frac{w(\lambda)}{2 \pi i} \int_{S} \frac{\log \rho(x) d x}{(\lambda-x) w^{+}(x)}-\frac{c_{g+1}}{2}-v(\lambda) w(\lambda)\right\} \\
& \times \exp \left\{2 \pi i \sum_{j=1}^{g} \theta_{j}(n) \Omega_{j}\left(\lambda^{(2)}\right)\right\} .
\end{aligned}
$$

Подведем итог. Мы показали, что при условии $\lambda_{j} \in\left(e_{2 j}, e_{2 j+1}\right)$, $j=1, \ldots, g$, существование решения задачи $(\mathscr{R})$ эквивалентно тому, что точки $\boldsymbol{\lambda}_{1}, \ldots, \boldsymbol{\lambda}_{g}$ являются решением проблемы обращения Якоби (В.12) со специальной правой частью и одновременно нашли явное представление (B.16)-(B.17) функции $\Psi(\boldsymbol{\lambda})$ для $\boldsymbol{\lambda} \in \mathfrak{R} \backslash \boldsymbol{\Gamma}$ и при нормировке $\Psi\left(\lambda^{(1)}\right) \Psi\left(\lambda^{(2)}\right)=\prod_{j=1}^{g}\left(\lambda-\lambda_{j}\right)$. Теперь уже нетрудно проверить непосредственно, что эти явные формулы сохраняют смысл, если при некотором $k \in\{1, \ldots, g\}$ точка $\lambda_{k}$ совпадает с одним из концов $k$-й лакуны $L_{k}$. Каждую такую точку $\boldsymbol{\lambda}_{k}$ будем считать как нулем предельного значения $\Psi^{+}(\mathbf{x})$, так и нулем предельного значения $\Psi^{-}(\mathbf{x}), \mathbf{x} \in \mathbf{\Gamma}$. При этом соглашении кусочно мероморфная на $\mathfrak{R}$ функция (В.16)-(В.17) дает решение задачи $(\mathscr{R})$ в общем случае. Отметим, что так как вес $\rho$ - голоморфная на $\boldsymbol{\Gamma}$ функция, то как функция $\Psi\left(\lambda^{(1)}\right), \lambda^{(1)} \in D^{(1)}$, так и функция $\Psi\left(\lambda^{(2)}\right), \lambda^{(1)} \in D^{(2)}$ голоморфно продолжаются через 
$\boldsymbol{\Gamma}$ на другой лист римановй поверхности. Так как, вообще говоря, $\rho \not \equiv 1$, то на $\boldsymbol{\Gamma}$ эти два голоморфных продолжения не совпадают.

Отметим, что классическому случаю $g=0$ и $S=[-1,1]$ соответствуют $\mathscr{F}(\boldsymbol{\lambda}) \equiv 1$ и

$$
D(\lambda ; \rho)=\exp \left\{A\left(\lambda^{(1)} ; \rho\right)\right\}=\exp \left\{\frac{\sqrt{\lambda^{2}-1}}{2 \pi} \int_{-1}^{1} \frac{\log \rho(x)}{\lambda-x} \frac{d x}{\sqrt{1-x^{2}}}\right\}
$$

- классическая функция Сегё. Случай, когда $S$ есть объединение непересекающихся интервалов вещественной оси, а вес $\rho$ такой, что $i \rho / \sqrt{h}>0$ и выполняются условия ортогональности

$$
\int_{S} \log \rho(x) d \Omega_{k}^{+}(x)=0, \quad k=1, \ldots, g,
$$

рассматривался Ахиезером [34]-[36], который для такого веса ввел оператор

$$
\exp \left\{\frac{\sqrt{h(\lambda)}}{2 \pi} \int_{S} \frac{\log \rho(x)}{\lambda-x} \frac{d x}{\sqrt{-h(x)}}\right\} .
$$

5. Докажем теперь, что если вес $\rho$ - марковский, т.е. $\rho(x) / \sqrt{-h(x)}>0$ на $S$, то проблема обращения Якоби (В.12) всегда имеет решение $\boldsymbol{\lambda}_{1}, \ldots, \boldsymbol{\lambda}_{g}$ такое, что все $\lambda_{j} \in\left[e_{2 j}, e_{2 j+1}\right]$. Так как такой дивизор $\boldsymbol{\lambda}_{1}+\cdots+\boldsymbol{\lambda}_{g}$ неспециальный, то отсюда сразу получим, что это решение единственно.

Итак, пусть все $\lambda_{j} \in\left[e_{2 j}, e_{2 j+1}\right]$. Нетрудно видеть, что при этом условии

$$
\int_{\infty(1)}^{\boldsymbol{\lambda}_{j}} d \Omega_{\ell}^{+}(\zeta)=\frac{1}{2} \sum_{k=1}^{g} \omega_{k}(\infty) B_{\ell k}+\frac{1}{2}-\frac{1}{2} B_{j \ell}-\frac{1}{2} \theta_{\ell j}+\int_{e_{2 j}}^{\boldsymbol{\lambda}_{j}} d \Omega_{\ell},
$$

где

$$
\theta_{\ell j}=\sum_{k=j+1}^{g} \delta_{\ell k}=\left\{\begin{array}{ll}
1, & \ell>j, \\
0, & \ell \leqslant j,
\end{array} \quad \sum_{j=1}^{g} \theta_{\ell j}=\ell-1, \quad 1 \leqslant \ell \leqslant g,\right.
$$

а путь интегрирования в последнем интеграле берется на первом листе по части отрезка $\left[e_{2 j}, e_{2 j+1}\right]$, если $\boldsymbol{\lambda}_{j}=\lambda_{j}^{(1)}$, и - на втором 
листе, если $\boldsymbol{\lambda}_{j}=\lambda_{j}^{(2)}$. Следовательно, из (В.18) получаем

$$
\begin{aligned}
& \sum_{j=1}^{g} \int_{\infty(1)}^{\boldsymbol{\lambda}_{j}} d \Omega_{\ell}^{+}(\zeta) \\
& \quad=\frac{g}{2} \sum_{k=1}^{g} \omega_{k}(\infty) B_{\ell k}+\frac{g}{2}-\frac{1}{2} \sum_{j=1}^{g} B_{j \ell}-\frac{1}{2} \sum_{j=1}^{g} \theta_{\ell j}+\sum_{j=1}^{g} \int_{e_{2 j}}^{\boldsymbol{\lambda}_{j}} d \Omega_{\ell} \\
& \quad=\frac{g}{2} \sum_{k=1}^{g} \omega_{k}(\infty) B_{\ell k}-\frac{1}{2} \sum_{j=1}^{g} B_{j \ell}+\frac{g-\ell+1}{2}+\sum_{j=1}^{g} \int_{e_{2 j}}^{\boldsymbol{\lambda}_{j}} d \Omega_{\ell}
\end{aligned}
$$

Так как вес $\rho$ - марковский, то $i \rho / w^{+}=\rho(x) / \sqrt{-h(x)}>0$ на $S$. Тем самым для выбранной выше ветви корня $\sqrt{h(\lambda)}$ (напомним, что $\sqrt{h(\lambda)}>0$ при $\left.\lambda>e_{2 g+2}\right)$ имеем: $\arg \rho(\zeta)=\arg \sqrt{h(\zeta)}-\pi / 2$, $\zeta \in S$; в частности, $\arg \rho(\zeta)=0$ при $\zeta \in \Delta_{g+1}$. Следовательно,

$$
\begin{aligned}
\int_{S} \log \rho(\zeta) d \Omega_{\ell}^{+}(\zeta) \\
\quad=\int_{S} \log |\rho(\zeta)| d \Omega_{\ell}^{+}(\zeta)+\sum_{k=1}^{g+1}(g+1-k) \pi i \int_{\Delta_{k}} d \Omega_{\ell}^{+}(\zeta) \\
\quad=\int_{S} \log |\rho(\zeta)| d \Omega_{\ell}^{+}(\zeta)+\frac{g-\ell+1}{2} \pi i
\end{aligned}
$$

Таким образом, система (В.12) эквивалентна следующей системе относительно точек $\boldsymbol{\lambda}_{j}=\boldsymbol{\lambda}_{j}(n), j=1, \ldots, g$ :

$$
\begin{aligned}
& \sum_{j=1}^{g} \int_{e_{2 j}}^{\boldsymbol{\lambda}_{j}} d \Omega_{\ell}(\boldsymbol{\zeta}) \\
& =-\frac{g}{2} \sum_{j=1}^{g} \omega_{j}(\infty) B_{\ell j}+\frac{1}{2} \sum_{j=1}^{g} B_{\ell j}+\frac{i}{\pi} \int_{S} \log |\rho(\zeta)| d \Omega_{\ell}^{+}(\zeta)- \\
& \quad-\sum_{j=1}^{g}(n-g) \omega_{j}(\infty) B_{\ell j}-\left(n_{\ell}-n_{g+1}\right)-\sum_{j=1}^{g} m_{j} B_{\ell j} \\
& \equiv-\frac{g}{2} \sum_{j=1}^{g} \omega_{j}(\infty) B_{\ell j}+\frac{1}{2} \sum_{j=1}^{g} B_{\ell j} \\
& \quad+\frac{i}{\pi} \int_{S} \log |\rho(\zeta)| d \Omega_{\ell}^{+}(\zeta)-\sum_{j=1}^{g}\left\{(n-g) \omega_{j}(\infty)\right\} B_{\ell j}
\end{aligned}
$$


$\ell=1, \ldots, g$. Нетрудно видеть, что из условия каноничности базиса дифференциалов $d \Omega_{\ell}$ (относительно заданных базисных циклов $\left.\left\{\mathbf{a}_{j}, \mathbf{b}_{j}\right\}\right)$ вытекает, что каждый дифференциал $d \Omega_{\ell}(x)$ вещественнозначен на $S$ и имеет ровно один нуль в каждом интервале $\left(e_{2 j-1}, e_{2 j}\right), j=1, \ldots, g, j \neq \ell$. В лакунах $\left(e_{2 j}, e_{2 j+1}\right), j=1, \ldots, g$, эти дифференциалы принимают чисто мнимые значения, отличные от нуля. Отсюда вытекает, во-первых, что все $B_{\ell j}$ чисто мнимые и, во-вторых, что и левая, и правая части (В.19) также чисто мнимые. Следовательно, поскольку первая часть (В.19) - точное равенство, то $n_{\ell}-n_{g+1}=0, \ell=1, \ldots, g$. Так как все точки $\boldsymbol{\lambda}_{j} \in \mathbf{L}_{j}$, то левая часть (В.19) равномерно ограничена при $n \rightarrow \infty$. Тем самым, ограничена и правая часть (В.19). Значит, равномерно ограничены величины $1 / 2-m_{j}-(n-g) \omega_{j}(\infty)-g \omega_{j}(\infty) / 2$. Поэтому $m_{j}=-\left[(n-g) \omega_{j}(\infty)\right]+\widetilde{m}_{j}(n)$, где $\widetilde{m}_{j}(n) \in \mathbb{Z}$ - равномерно ограничены; здесь [·] - целая часть числа. Кроме того, матрица $\left\|B_{\ell j}\right\|$ невырождена. Следовательно, существуют такие величины $\rho_{1}, \ldots, \rho_{g}$, что все $\rho_{j} \in \mathbb{R}$ и

$$
\sum_{j=1}^{g} \rho_{j} B_{\ell j}=\frac{i}{\pi} \int_{S} \log |\rho(\zeta)| d \Omega_{\ell}^{+}(\zeta), \quad \ell=1, \ldots, g .
$$

Значит, (В.19) записывается в виде:

$$
\sum_{j=1}^{g} \int_{e_{2 j}}^{\boldsymbol{\lambda}_{j}} d \Omega_{\ell}(\boldsymbol{\zeta})=\sum_{j=1}^{g} y_{j} B_{\ell j} \quad(\bmod \text { периодов }),
$$

где

$$
\begin{aligned}
y_{j} & =\frac{1}{2}-m_{j}-\frac{g}{2} \omega_{j}(\infty)+\rho_{j}-(n-g) \omega_{j}(\infty) \quad(\bmod 1) \\
& =\frac{1}{2}-\frac{g}{2} \omega_{j}(\infty)+\frac{1}{2}+\rho_{j}-(n-g) \omega_{j}(\infty) \quad(\bmod 1) \\
& =\frac{1}{2}-\frac{g}{2} \omega_{j}(\infty)+\rho_{j}-\left\{(n-g) \omega_{j}(\infty)\right\} \quad(\bmod 1),
\end{aligned}
$$

$y_{j} \in[0,1)$. В отличие от случая общего отображения Абеля (А.1), в соотношениях (В.20) точки $\boldsymbol{\lambda}_{1}, \ldots, \boldsymbol{\lambda}_{g}$ уже упорядочены тем, что интегрирование ведется от левого края лакуны $e_{2 j}$ до соответствующей точки $\boldsymbol{\lambda}_{j}$ и $\lambda_{1}<\cdots<\lambda_{g}$. Тем самым, соотношения (В.20) задают отображение вещественного $g$-мерного тора $\mathbb{T}^{g}=\mathbf{L}_{1} \times \cdots \times \mathbf{L}_{g}$ в тор $\mathbb{T}^{g}=[0,1) \times \cdots \times[0,1)$. Это отображение 
непрерывно и инъективно, следовательно, оно биективно (см. п. 4 приложения A, а также [37, гл. X]). Тем самым, при любых вещественных $y_{k} \in[0,1)$ система (В.20) имеет (единственное) решение $\boldsymbol{\lambda}_{1}, \ldots, \boldsymbol{\lambda}_{g}$ такое, что все $\boldsymbol{\lambda}_{j} \in \mathbf{L}_{j}$.

6. Рассмотрим в Jас $\mathfrak{R}$ множество точек с координатами

$$
x_{k}=x_{k}^{0}, \quad y_{k}=\tau_{k}, \quad k=1, \ldots, g, \quad \text { где } \tau_{k} \in[0,1),
$$

изоморфное $g$-мерному вещественному тору $\mathbb{T}^{g}$. Пусть $\mathbb{S}=\mathbb{S}\left(x^{0}\right)$ - соответствующее ему множество в $S^{g} \mathfrak{R}$ :

$$
\begin{aligned}
\mathbb{S}= & \left\{d=\boldsymbol{\lambda}_{1}+\cdots+\boldsymbol{\lambda}_{g}:\right. \\
& \left.\sum_{j=1}^{g} \Omega_{k}\left(\boldsymbol{\lambda}_{j}\right) \equiv x_{k}^{0}+\sum_{j=1}^{g} \tau_{j} B_{k j}, \tau_{j} \in[0,1), k=1, \ldots, g\right\} .
\end{aligned}
$$

Если координаты $x_{k}^{0}$ дивизора $d^{0}=\boldsymbol{\lambda}_{1}^{0}+\cdots+\boldsymbol{\lambda}_{g}^{0}$ таковы, что всякий дивизор $d$ из $\mathbb{S}$ - неспециальный, то каждый дивизор $d \in \mathbb{S}$ однозначно определяется заданными $\tau_{j} \in[0,1): d=d\left(\tau_{1}, \ldots, \tau_{g}\right)$, т.е. $\mathbb{S}$ также изоморфно $\mathbb{T}^{g}$. Пусть

$$
\begin{aligned}
& \mathbb{S}_{1}=\left\{d \in \mathbb{S}: \tau_{j}=y^{0}+t \omega_{j}(\infty) \quad(\bmod 1), t \in \mathbb{R}_{+}, j=1, \ldots, g\right\}, \\
& \mathbb{S}_{0}=\left\{d \in \mathbb{S}: \tau_{j}=y^{0}+n \omega_{j}(\infty) \quad(\bmod 1), n \in \mathbb{N}, j=1, \ldots, g\right\},
\end{aligned}
$$

$\mathbb{S}_{0} \subset \mathbb{S}_{1} \subset \mathbb{S} ; \mathbb{S}_{1}=\left\{d(t)=\boldsymbol{\lambda}_{1}(t)+\cdots+\boldsymbol{\lambda}_{g}(t)\right\}-$ прямолинейная обмотка тора $\mathbb{S}, \mathbb{S}_{0}=\left\{d(n)=\boldsymbol{\lambda}_{1}(n)+\cdots+\boldsymbol{\lambda}_{g}(n)\right\}-$ счетное множество. Движение дивизора $d(t)=\boldsymbol{\lambda}_{1}(t)+\cdots+\boldsymbol{\lambda}_{g}(t)$ на тоpe $\mathbb{S}-$ квазипериодическое с группой периодов $\omega_{1}(\infty), \ldots, \omega_{g}(\infty)$ (см., например, [38], глава 10, §51). С точки зрения изучения асимптотического поведения нулей $Q_{n}$ и $R_{n}$ нас интересует множество предельных точек $\mathbb{S}_{0}^{\prime}$. Отметим следующий хорошо известный факт [38, гл. 10, §51]: если величины $\omega_{1}(\infty), \ldots, \omega_{g}(\infty)$ рационально независимы, то $\mathbb{S}_{1}^{\prime}=\mathbb{S}$, т.е. траектория $\mathbb{S}_{1}$ дивизора $d(t)$ всюду плотна в $\mathbb{S}$; если же $\omega_{1}(\infty), \ldots, \omega_{g}(\infty)$ и 1 рационально независимы $^{9}$, то и $\mathbb{S}_{0}^{\prime}=\mathbb{S}$.

Функции $\lambda_{j}=\lambda_{j}(t)-$ вещественно-аналитические функции переменной $t \in \mathbb{R}$, поэтому для $d \in \mathbb{S}_{1}$ получаем:

$$
\sum_{j=1}^{g} \Omega_{k}^{\prime}\left(\boldsymbol{\lambda}_{j}\right) \dot{\lambda}_{j}=\sum_{j=1}^{g} \omega_{j}(\infty) B_{j k}, \quad k=1, \ldots, g,
$$

\footnotetext{
${ }^{9} \mathrm{~B}$ силу соотношения $\omega_{1}(\infty)+\cdots+\omega_{g+1}(\infty)=1$ это условие эквивалентно тому, что $\omega_{1}(\infty), \ldots, \omega_{g+1}(\infty)$ рационально независимы.
} 
где $\dot{\lambda}_{k}=d \lambda_{k} / d t$. Рассмотрим (В.22) как линейную систему относительно $\dot{\lambda}_{j}$. Нетрудно видеть, что

$$
\operatorname{det}\left\|\Omega_{k}^{\prime}\left(\boldsymbol{\lambda}_{j}\right)\right\|=\frac{\text { const }}{\prod_{j=1}^{g} w\left(\boldsymbol{\lambda}_{j}\right)} v\left(\lambda_{1}, \ldots, \lambda_{g}\right),
$$

где const зависит только от точек $e_{1}, \ldots, e_{2 g+2}, V$ - определитель Вандермонда от $g$ переменных. Пусть $D_{k}$ - определитель матрицы, полученной из матрицы системы (В.22) заменой $k$-го столбца на столбец правых частей. С помощью соотношения (А.5) получаем:

$$
D_{k}=\mathrm{const} \cdot \int_{e_{2 g+2}}^{+\infty} \frac{V_{k}\left(\lambda_{1}, \ldots, x, \ldots, \lambda_{g}\right)}{\prod_{j \neq k} w\left(\boldsymbol{\lambda}_{j}\right)} \frac{d x}{w(x)}
$$

где const - та же, что и в (В.23), путь интегрирования - луч от $e_{2 g+2}$ до $+\infty, V_{k}$ - определитель Вандермонда от $g$ переменных, в котором $\lambda_{k}$ заменено на $x$. Разрешая теперь систему (В.22) относительно величин $\dot{\lambda}_{j}$ и используя только что выведенные формулы (В.23)-(В.24), а также явную формулу для определителя Вандермонда, получаем динамическую систему (26). Система Дубровина [39] (см. также [37], глава VIII, формула (8.2.15))

$$
\dot{\lambda}_{k}= \pm \frac{2 \sqrt{-h\left(\lambda_{k}\right)}}{\prod_{j \neq k}\left(\lambda_{k}-\lambda_{j}\right)}, \quad k=1, \ldots, g
$$

описывающая динамику нулей конечно-зонных решений уравнения Шрёдингера, получается из (26) заменой сомножителя $\left(\lambda-e_{2 g+2}\right)$ на $\left(\lambda / e_{2 g+2}-1\right)$ и последующим (формальным) предельным переходом при $e_{2 g+2} \rightarrow \infty$.

Положим

$$
\vec{\Omega}(\lambda)=\left(\Omega_{1}(\lambda), \ldots, \Omega_{g}(\lambda)\right)^{T}, \quad \vec{\omega}(\lambda)=\left(\omega_{1}(\lambda), \ldots, \omega_{g}(\lambda)\right)^{T},
$$

где $\lambda \in D, \omega_{j}(\lambda)=\omega\left(\lambda ; \Delta_{j}, D\right)$. Нетрудно проверить, что справедливо следующее представление

$$
\operatorname{Re}\left\{\mathbf{B}^{-1} \vec{\Omega}(\lambda)\right\}=-\frac{1}{2} \vec{\omega}(\lambda), \quad \lambda \in D .
$$


$\mathrm{C}$ учетом этой формулы соотношения (26) для дивизора $d(t)=$ $\boldsymbol{\lambda}_{1}(t)+\cdots+\boldsymbol{\lambda}_{g}(t) \in \mathbb{S}_{1}$ переписываются в терминах гармонических мер так:

$$
\sum_{j=1}^{g} \varepsilon_{j} \omega_{k}\left(\lambda_{j}\right)=(g-2 t) \omega_{k}(\infty)+\rho_{k} \quad(\bmod 2)
$$

где $\varepsilon_{j}= \pm 1$ в зависимости от точки $\boldsymbol{\lambda}_{j}=\left(\lambda_{j}, \pm\right)$, а для величин $\rho_{k}$ справедливо представление

$$
\rho_{k}=\frac{1}{\pi} \int_{S} \log |\rho(\zeta)| \frac{\partial \omega_{k}(\zeta)}{\partial n_{\zeta}^{+}} d \zeta, \quad k=1, \ldots, g
$$

здесь $\partial / \partial n_{\zeta}$ означает производную по внутренней нормали к границе области $D$. В частности, если при переходе от $t=n \mathrm{k} t=n+1$ знаки всех величин $\varepsilon_{j}$ сохраняются, то имеем

$$
\sum_{j=1}^{g} \varepsilon_{j} \Delta \omega_{k}\left(\lambda_{j}\right)=-2 \omega_{k}(\infty) \quad(\bmod 2)
$$

(ср. [29], лемма 3). Соотношения (В.26) означают, что движение дивизора $d(t)=\boldsymbol{\lambda}_{1}(t)+\cdots+\boldsymbol{\lambda}_{g}(t)$ на торе $\mathbf{L}_{1} \times \cdots \times \mathbf{L}_{g}$ происходит с постоянной "угловой скоростью" (см. ниже п. 7).

Подчеркнем, что здесь фактически доказано, что система сравнений относительно величин $\varepsilon_{j}= \pm 1$ и $\lambda_{j}$

$$
\begin{aligned}
\sum_{j=1}^{g} \varepsilon_{j} \omega_{k}\left(\lambda_{j}\right)= & (g-2 n) \omega_{k}(\infty) \\
& -\frac{2}{\pi} \int_{S} \log |\rho(\zeta)| \frac{\partial \omega_{k}(\zeta)}{\partial n_{\zeta}^{+}} d \zeta \quad(\bmod 2), k=1, \ldots, g
\end{aligned}
$$

всегда имеет единственное решение $\lambda_{1}, \ldots, \lambda_{g}$, притом такое, что все $\lambda_{j} \in\left[e_{2 j}, e_{2 j+1}\right]$.

Соотношение (В.25) приводит к следующей формуле для $y$ координат $\tau_{k}, k=1, \ldots, g$, дивизора $d=\boldsymbol{\lambda}_{1}+\cdots+\boldsymbol{\lambda}_{g} \in \mathbb{S}$ :

$$
\tau_{k}=-\frac{1}{2} \sum_{j=1}^{g} \varepsilon_{j} \omega_{k}\left(\lambda_{j}\right) \quad(\bmod 1)
$$


и следующей связи между комплексными гармоническими мерами $\omega_{k}(\lambda)+i \omega_{k}^{*}(\lambda)$ и абелевыми интегралами:

$$
\Omega_{j}(\lambda)=-\frac{1}{2} \sum_{k=1}^{g} B_{k j}\left(\omega_{k}(\lambda)+i \omega_{k}^{*}(\lambda)\right), \quad \lambda \in D .
$$

7. Напомним геометрический смысл гармонической меры (см., например, [40]). В этом пункте предполагается, что $D$ - односвязная ограниченная область в $\mathbb{C}$, граница $\partial D$ которой - аналитическая кривая. Пусть $\ell=\ell\left(\zeta_{1}, \zeta_{2}\right) \subset \partial D-$ открытая дуга на $\partial D$; см. рис. 2 .

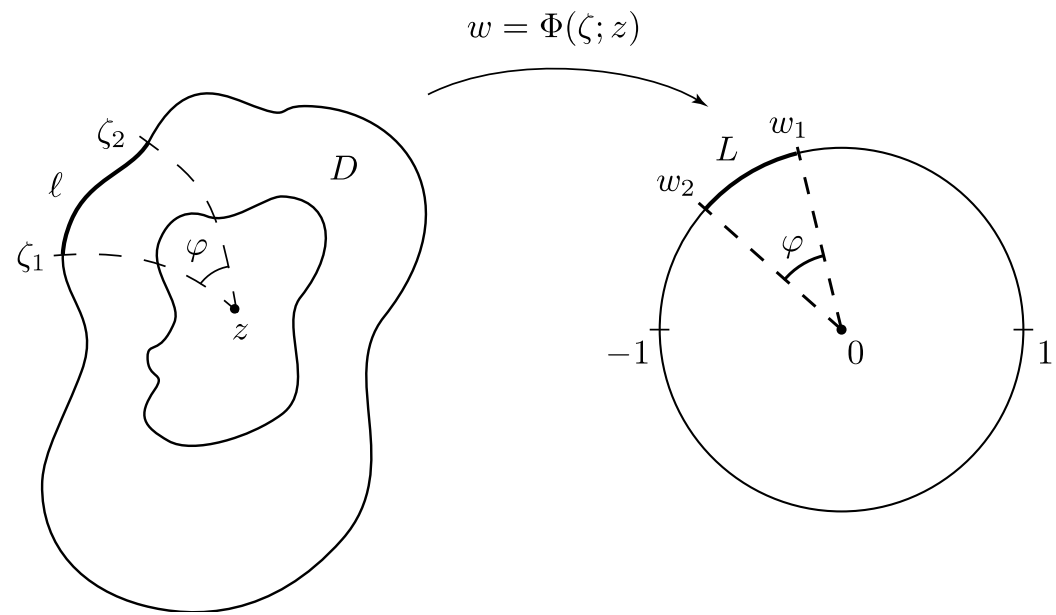

Рис. 2. Функция $w=\Phi(\zeta ; z)=\exp \{-G(\zeta, z)\}$ отображает конформно и однолистно область $D$ на внутренность единичного круга $\mathbb{D}:|w|<1$ так, что точка $\zeta=z$ переходит в точку $w=0$. При этом по принципу соответствия границ, дуге $\ell=\ell\left(\zeta_{1}, \zeta_{2}\right) \subset \partial D$ соответствует дуга $L=L\left(w_{2}, w_{1}\right) \subset \partial \mathbb{D}, w_{j}=\exp \left\{-i g^{*}\left(\zeta_{j}, z\right)\right\}$.

Функция $\omega(z)=\omega(z ; \ell, D)-$ ограниченная гармоническая функция в области $D$, предельные значения которой удовлетворяют следующим условиям:

$$
\lim _{z \rightarrow \zeta} \omega(z)= \begin{cases}1, & \zeta \in \ell \\ 0, & \zeta \in \partial D \backslash \bar{\ell}\end{cases}
$$


Зафиксируем точку $z \in D$. Пусть $g(\zeta, z), \zeta \in D,-$ функция Грина области $D$ с особенностью в точке $\zeta=z, g^{*}(\zeta, z)$ - гармонически сопряженная ей функция, $G(\zeta, z)=g(\zeta, z)+i g^{*}(\zeta, z)-($ многозначная) комплексная функция Грина. Функция $w=\Phi(\zeta ; z)=$ $\exp \{-G(\zeta, z)\}$ отображает конформно и однолистно область $D$ на внутренность единичного круга $\mathbb{D}:|w|<1$ так, что точка $\zeta=z$ переходит в точку $w=0$. При этом по принципу соответствия границ, дуге $\ell \subset \partial D$ соответствует дуга $L=L\left(w_{2}, w_{1}\right) \subset \partial \mathbb{D}$, $w_{j}=\exp \left\{-i g^{*}\left(\zeta_{j}, z\right)\right\}$. Напишем для функции $\omega(z)$ формулу Грина:

$$
\omega(z)=\frac{1}{2 \pi} \int_{\ell} \frac{\partial g(\zeta, z)}{\partial n_{\zeta}} d s_{\zeta},
$$

где $\partial / \partial n_{\zeta}-$ производная по внутренней нормали к $\ell$, и воспользуемся тем, что $\partial g(\zeta, z) / \partial n_{\zeta}=\partial g^{*}(\zeta, z) / \partial t_{\zeta}$, где $\partial / \partial t_{\zeta}-$ производная по касательной к кривой $\ell$ в точке $\zeta$, причем вектор касательной $t_{\zeta}$ получается из вектора нормали $n_{\zeta}$ поворотом против часовой стрелки на угол $\pi / 2$. Тогда (В.28) примет вид:

$$
\begin{aligned}
\omega(z) & =\frac{1}{2 \pi} \int_{\ell} \frac{\partial g^{*}(\zeta, z)}{\partial t_{\zeta}} d s_{\zeta}=\frac{g^{*}\left(\zeta_{2}, z\right)-g^{*}\left(\zeta_{1}, z\right)}{2 \pi} \\
& =\frac{\arg w_{2}-\arg w_{1}}{2 \pi}=\frac{\varphi}{2 \pi},
\end{aligned}
$$

где $\varphi=\arg w_{2}-\arg w_{1}-$ угол, под которым дуга $\ell$ "видна" из точки $z$.

Рассмотрим теперь случай многосвязной области $D$, соответствующий ситуации, когда в носителе меры $\mu$ имеется конечное число $g \geqslant 1$ лакун $\left(\alpha_{j}, \beta_{j}\right), j=1,2, \ldots, g$, т.е. $\operatorname{supp} \mu=$ $[-1,1] \backslash \bigcup_{j=1}^{g}\left(\alpha_{j}, \beta_{j}\right)$. Пусть $g(z, \infty)=g_{D}(z, \infty)-$ функция Грина области $D, g^{*}(z, \infty)$ - гармонически сопряженная ей функция, $G(z, \infty)=g(z, \infty)+i g^{*}(z, \infty)$ - многозначная комплексная функция Грина. В верхней полуплоскости $\Pi_{+}: \operatorname{Im} z>0$ эта функция однозначна. Нормируем ее условием $g^{*}(1, \infty)=0$. Тогда $g^{*}(-1, \infty)=\pi$ и функция $w=\Phi(z)=\exp \{-G(z, \infty)\}$ отображает верхнюю полуплоскость П + конформно и однолистно на нижний полукруг $K_{-}:|w|<1, \operatorname{Im} w<0$, так, что $\Phi(1)=1, \Phi(\infty)=0$, $\Phi(-1)=-1$. При этом отрезки $\Delta_{j}=\left[\beta_{j-1}, \alpha_{j}\right], j=1, \ldots, g+1$, где $\beta_{0}=-1, \alpha_{g+1}=1$, переходят в дуги $\ell_{j}$ единичной полуокружности $w=e^{i \varphi},-\pi \leqslant \varphi \leqslant 0$, а лакуны $\left(\alpha_{j}, \beta_{j}\right), j=1,2, \ldots, g,-$ 
в радиальные отрезки $I_{j}$ длины $\left|I_{j}\right|<1$, выходящие из концов соответствующих дуг; см. рис. 3.

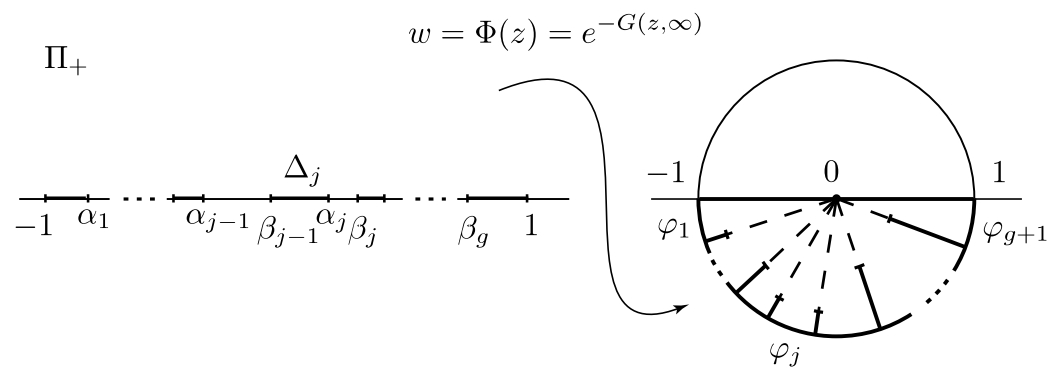

Рис. 3. Функция $w=\Phi(z)=\exp \{-G(z, \infty)\}$ отображает верхнюю полуплоскость $\Pi_{+}$конформно и однолистно на нижний полукруг $K_{-}:|w|<1, \operatorname{Im} w<0$, так, что $\Phi(1)=1, \Phi(\infty)=0, \Phi(-1)=-1$. При этом отрезки $\Delta_{j}=\left[\beta_{j-1}, \alpha_{j}\right], j=1, \ldots, g+1$, где $\beta_{0}=-1$, $\alpha_{g+1}=1$, переходят в дуги $\ell_{j}$ единичной полуокружности $w=$ $e^{i \varphi},-\pi \leqslant \varphi \leqslant 0$, а лакуны $\left(\alpha_{j}, \beta_{j}\right), j=1,2, \ldots, g,-$ в радиальные отрезки $I_{j}$ длины $\left|I_{j}\right|<1$, выходящие из концов соответствующих дуг. Величина $j$-го угла $\varphi_{j}$ равна $\pi \omega_{j}(\infty)$.

Используя формулу Грина (В.28) для гармонической меры и соотношение $\partial g(\zeta, \infty) / \partial n_{\zeta}=\partial g^{*}(\zeta, \infty) / \partial t_{\zeta}$, нетрудно вычислить величину $j$-го угла $\varphi_{j}$ :

$$
\varphi_{j}=g^{*}\left(\beta_{j-1}, \infty\right)-g^{*}\left(\alpha_{j}, \infty\right) \pi \omega_{j}(\infty), \quad j=1, \ldots, g+1,
$$

где $\omega_{j}(\infty)=\omega\left(\infty ; \Delta_{j}, D\right)$. Тем самым, длина дуги $\bigcup_{k=j+1}^{g+1} \ell_{k}$ равна $\theta_{j} *=\sum_{k=j+1}^{g+1} \varphi_{k}, j=g, \ldots, 0 ; \quad \theta_{0}=\pi\left(\omega_{1}(\infty)+\cdots+\omega_{g+1}(\infty)\right)=\pi$.

Так как в каждой лакуне $\left(\alpha_{j}, \beta_{j}\right)$ располагается ровно по одному нулю $\zeta_{j}$ производной комплексной функции Грина, то длины соответствующих лакунам радиальных отрезков, исходящих из точек $w_{j}=e^{-\theta_{j}}, j=1, \ldots, g$, вычисляются по следующей формуле:

$$
\left|I_{j}\right|=1-\Phi\left(\zeta_{j}\right) e^{\theta_{j}}=1-e^{-g\left(\zeta_{j}, \infty\right)}<1
$$


Непосредственно из (В.29) вытекает, что случай равных углов $\varphi_{j}$ в точности соответствует случаю равных гармонических мер $\omega_{j}(\infty)$; см. [41].

\section{Приложение C}

Задача, рассматриваемая Чебышёвым в [1], состоит в следующем. Пусть некоторая функция $F$ задана на $\mathbb{R}$ в конечном (притом достаточно большом) числе узлов $x_{j}, j=1,2, \ldots, N$. Требуется по значениям $F_{j}=F\left(x_{j}\right)$ в этих узлах построить приближенное представление функции $F$ полиномом фиксированной "малой" степени $n, n \ll N$, которое является в определенном смысле оптимальным. Оптимальность представления понимается как минимизация среднеквадратичного уклонения значений полинома в узлах $x_{j}$ от значений функции $F_{j}$, притом - с некоторыми неотрицательными весами $\theta_{j}^{2} \geqslant 0, \theta_{1}^{2}+\cdots+\theta_{N}^{2}=1$, заданными в точках $x_{j}$. Поставленная задача была решена Чебышёвым следующим образом. Рассмотрим марковскую функцию ${ }^{10}$ (ср. с (1))

$$
f(\lambda)=\sum_{j=1}^{N} \frac{\theta_{j}^{2}}{\lambda-x_{j}}, \quad \text { где } \lambda \neq x_{j} .
$$

Разложим ее в (конечную) непрерывную дробь:

$$
f(\lambda)=\frac{1}{\lambda-b_{1}-\frac{a_{1}^{2}}{\lambda-b_{2}-\frac{a_{2}^{2}}{\lambda-b_{N-1}-\frac{a_{N-1}^{2}}{\lambda-b_{N}}}} .}
$$

${ }^{10}$ Отметим, что как Чебышёв, так и Марков при выводе формальных соотношений пользовались обозначением $\sum_{t} \frac{\theta^{2}(t)}{\lambda-t}$, достаточно свободно, трактуя в зависимости от контекста в этом выражении знак $\sum_{t}$ и как знак суммирования для $t=x_{j}$ и $j=1,2, \ldots, N$ с весом $\theta_{j}^{2}=\theta^{2}\left(x_{j}\right)$, и как знак интегрирования при $t \in[a, b] \Subset \mathbb{R}$ с весовой функцией $\theta^{2}(t)$. 
Тогда $n$-я подходящая дробь $P_{n} / O_{n}, n=1, \ldots, N$,

$$
\frac{P_{n}}{Q_{n}}(\lambda)=\frac{1}{\lambda-b_{1}-\frac{a_{1}^{2}}{\lambda-b_{2}-\frac{a_{2}^{2}}{\lambda-b_{n-1}-\frac{a_{n-1}^{2}}{\lambda-b_{n}}}}}
$$

к непрерывной дроби (C.1) обладает следующим характеристическим свойством в классе $\mathscr{R}_{n}$ рациональных дробей вида $r=p / q$, где $\operatorname{deg} p, \operatorname{deg} q \leqslant n, q \not \equiv 0$ :

$$
f(\lambda)-\frac{P_{n}}{Q_{n}}(\lambda)=O\left(\frac{1}{\lambda^{2 n+1}}\right), \quad \lambda \rightarrow \infty .
$$

Многочлены $Q_{n}$ имеют степень ровно $n$, удовлетворяют условиям ортогональности с весами $\left\{\theta_{j}^{2}\right\}_{j=1, \ldots, N}$ :

$$
\sum_{j=1}^{N} Q_{n}\left(x_{j}\right) x_{j}^{k} \theta_{j}^{2}=0, \quad k=0,1, \ldots, n-1,
$$

нормировкой $Q_{n}(\lambda)=\lambda^{n}+\cdots$ определены однозначно и удовлетворяют следующим трехчленным рекуррентным соотношениям:

$$
\begin{gathered}
Q_{n}(\lambda)=\left(\lambda-b_{n}\right) Q_{n-1}(\lambda)-a_{n-1}^{2} Q_{n-2}(\lambda), \quad n=2,3, \cdots, N \\
Q_{0}(\lambda) \equiv 1, \quad Q_{1}(\lambda)=\lambda-b_{1} .
\end{gathered}
$$

Многочлены $P_{n}$ - степени $n-1$, определяются непосредственно по $Q_{n}$ :

$$
P_{n}(\lambda)=\sum_{j=1}^{N} \frac{Q_{n}(\lambda)-Q_{n}\left(x_{j}\right)}{\lambda-x_{j}} \theta_{j}^{2}
$$

и называются многочленами второго рода.

Пусть $q_{n}(\lambda)=k_{n} Q_{n}(\lambda)=k_{n} \lambda^{n}+\cdots, k_{n}>0, n=0,1, \ldots, N,-$ соответствующие узлам $\left\{x_{j}, \theta_{j}^{2}\right\}_{j=1, \ldots, N}$ ортонормированные многочлены:

$$
\sum_{j=1}^{N} q_{n}\left(x_{j}\right) q_{m}\left(x_{j}\right) \theta_{j}^{2}=\delta_{n m} .
$$


Для них выполняется следующее трехчленное рекуррентное соотношение:

$$
a_{n} q_{n}(\lambda)=\left(\lambda-b_{n}\right) q_{n-1}(\lambda)-a_{n-1} q_{n-2}(\lambda)
$$

где $n=2,3, \ldots, N, q_{0}(\lambda) \equiv 1, a_{1} q_{1}(\lambda)=\lambda-b_{1}$. В [1] Чебышёв сопоставил функции $F$ ее "разложение" по ортонормированной системе $\left\{q_{n}(\lambda)\right\}_{n=0}^{N}$ :

$$
F(\lambda) \sim \sum_{k=1}^{N} A_{k} q_{k}(\lambda), \quad A_{k}=A_{k}(F)=\sum_{j=1}^{N} F\left(x_{j}\right) q_{k}\left(x_{j}\right) \theta_{j}^{2} .
$$

Тем самым, $A_{k}(F)$ есть фактически $k$-й коэффициент Фурье ${ }^{11}$ функции $F$ по системе $\left\{q_{k}(x)\right\}_{k=0}^{N}$. Отсюда уже легко следует, что искомый оптимальный многочлен заданной степени $n$ есть соответствующая разложению (С.6) n-я частичная сумма

$$
S_{n}(\lambda)=\sum_{k=1}^{n} A_{k} q_{k}(\lambda)
$$

наименее уклоняющаяся от $F$ в среднем квадратичном с заданными весами $\left\{\theta^{2}\left(x_{j}\right)\right\}_{j=1}^{N}$. В [1] Чебышёв нашел и другое подставление для оптимального многочлена, использующее так называемое воспроизводящее ядро Сегё. Вместе с тем он особо отмечает, что найденное им представление (С.7) обладает тем преимуществом, что при необходимости перейти от приближения степени $n$ к приближению степени $n+1$ не надо искать заново $(n+1)$-й оптимальный многочлен, а нужно лишь к $n$-му добавить одно слагаемое $A_{n+1} q_{n+1}(\lambda)$.

${ }^{11}$ Подчеркнем, что в [1] Чебышёв отмечает аналогию с обычными коэффициентами Фурье совершенно отчетливо. 


\section{Список литературы}

[1] П.Л. Чебышёв, “О непрерывных дробях", Ученые записки Имп. Академии Наук, III (1855), С. 636-664 (На франц. языке: Tchébycheff P. Sur les fractions continues. - Journ. de math. pures et appl. Sér. 2, 1858, Vol. 3, P. 289-323; Полное собрание сочинений. Т. ІІ. М.-Л.: Изд-во АН СССР, 1948, С. 103-126)

[2] Н. И. Ахиезер, “Чебышёвское направление в теории функций”, $M a$ тематика XIX века, ред. А. Н. Колмогоров и А. П. Юшкевич, Наука, М., 1987, 9-79, MR 0916262.

[3] Т.И. Стильтьес, Исследования о непрерывных дробях, ОНТИ, Харьков-Киев, 1936.

[4] P. Tchébycheff, "Sur le dévelopment des fonctions à une seule variable", Bull. Acad. Sci. St.-Pétersb. Cl. Phys.-Math., 1 (1860), 193-200, (На русском языке: П. Л. Чебышёв, О разложении функций одной переменной. Полное собрание сочинений, Т. II. М.-Л.: Изд-во АН CCCP, 1948, С. 335-341).

[5] Л. Д. Фаддеев, О. А. Якубовский, Лекиии по квантовой механике для студентов-математиков, РХД, Москва-Ижевск, 2001.

[6] Г. Сегё, Ортогональные многочлень, Физматгиз, М., 1962.

[7] Е. М. Никишин, “Дискретный оператор Штурма-Лиувилля и некоторые задачи теории функций”, Труды семинара имени И. Г. Петровского., 10 (1984), 3-77, MR 778879, Zbl 0573.34023.

[8] Damanik D., Killip R., and Simon B., "Necessary and sufficient conditions in the spectral theory of Jacobi matrices and Schrödinger operators", arXiv: math.SP/0309206 Vol. 1, Sept. 12, 2003, MR 2041649, ADS 2003math.....9206D.

[9] K.M. Case, "Orthogonal polynomials from the viewpoint of scattering theory", J. Math. Phys., 15:12 (1974), 2166-2174, doi 10.1063/1.1666597, MR 353860, Zbl 0288.42009.

[10] J. S. Geronimo and K. M. Case, "Scattering theory and polynomials orthogonal on the real line", Trans. Amer. Math. Soc., 258:2 (1980), 467-494, doi 10.2307/1998068, MR 558185, Zbl 0436.42018.

[11] B. Simon, "The classical moment problem as a self-adjoint finite difference operator", Adv. Math., 137 (1998), 82-203, doi 10.1006/aima.1998.1728, MR 1627806, Zbl 0910.44004.

[12] А. И. Аптекарев, Е. М. Никишин, "Задача рассеяния для дискретного оператора Штурма-Лиувилля", Матем. сб., 121(163):3(7) (1983), 327-358, Mi sm2205, MR 708000, Zbl 0527.34024.

[13] А. И. Аптекарев, "Асимптотические свойства многочленов, ортогональных на системе контуров, и периодические движения цепочек Тода", Матем. сб., 125(167):2(10) (1984), 231-258, Mi sm2080, MR 764479. 
[14] В.А. Калягин, "Аппроксимации Эрмита-Паде и спектральный анализ несимметричных разностных операторов", Матем. сб., 185:6 (1994), 79-100, Mi sm903, Zbl 0840.47026.

[15] D. Damanik and B. Simon, "Jost functions and Jost solutions for Jacobi matrices, I. A necessary and sufficient condition for Szegö asymptotics", arXiv: math.SP/0502486 Vol. 1, Feb. 23, 2005, MR 2221136, ADS 2005math.....2486D.

[16] R. Killip and B. Simon, "Sum rules for Jacobi matrices and their applications to spectral theory", Ann. of Math. (2), 158 (2003), 253321, MR 1999923, Zbl 1050.47025.

[17] I. Egorova and L. Golinskii, "Discrete spectrum for complex perturbations of periodic Jacobi matrices", J. Difference Equ. Appl., 11:14 (2005), 1185-1203, MR 2182247.

[18] А. А. Гончар, “О сходимости аппроксимаций Паде для некоторых классов мероморфных функций", Матем. сб., 97 (139) (1975), 607-629, Zbl 0341.30029.

[19] С. П. Суетин, "Спектральные свойства некоторого класса дискретных операторов Штурма-Лиувилля”, УМН, 61:2 (2006), 171-172.

[20] А. А. Гончар, "О равномерной сходимости диагональных аппроксимаций Паде”, Матем. сб., 118 (160):4 (1982), 535-556, Mi sm2831, MR 671708, Zbl 0529.41016.

[21] Е. А. Рахманов, “Об асимптотике отношения ортогональных многочленов", Матем. сб., 103(145):2(6) (1977), 237-252, Mi sm2806, MR 445212, Zbl 0373.30034.

[22] С. П. Суетин, "Об асимптотических свойствах полюсов диагональных аппроксимаций Паде для некоторых обобщений марковских функций", Матем. сб., 193:12 (2002), 103-155, MR 1992106.

[23] А. А. Гончар, С. П. Суетин, "Об аппроксимациях Паде мероморфных функций марковского типа", Современные проблемы математики, 5, Математический ин-т им. В. А. Стеклова РАН, Москва, 2004, 3-65.

[24] С. П. Суетин, "Об интерполяционных свойствах диагональных аппроксимаций Паде эллиптических функций”, УМН, 59:4 (2004), 201-202, MR 2106654.

[25] С. П. Суетин, "О равномерной сходимости диагональных аппроксимаций Паде для гиперэллиптических функций”, Матем. сб., 191:9 (2000), 81-114, Mi sm508, MR 1805599, Zbl 0980.41015.

[26] С. П. Суетин, "О динамике "блуждающих" нулей полиномов, ортогональных на нескольких отрезках", УМН, 57:2 (2002), 199-200, Mi rm507, MR 1918205, Zbl 02076326.

[27] С.П. Суетин, "Аппроксимации Паде и эффективное аналитическое продолжение степенного ряда", УМН, 57:1 (2002), 45-142, Mi rm475, MR 1914542, Zbl 1056.41005. 
[28] J. Nuttall, "Pade polynomial asymptotics from a singular integral equation", Constr. Approx., 6:2 (1990), 157-166, doi 10.1007/BF01889355, MR 1036606, Zbl 0685.41014.

[29] Е.А. Рахманов, "О сходимости диагональных аппроксимаций Паде", Матем. сб., 104 (146):2 (1977), 271-291, MR 1687726, $\mathrm{Zbl} 0376.30011$.

[30] H. Widom, "Extremal polynomials associated with a system of curves in the complex plane", Adv. Math., 3 (1969), 127-232, doi 10.1016/0001-8708(69)90005-X, MR 239059, Zbl 0183. 07503.

[31] Э.И. Зверович, "Краевые задачи теории аналитических функций в гёльдеровских классах на римановых поверхностях", УМH, 26:1 (1971), 113-180, MR 409841.

[32] Дж. Спрингер, Введение в теорию римановых поверхностей, ИЛ, M., 1960, MR 0122987.

[33] Б.А. Дубровин, "Тэта-функции и нелинейные уравнения", УМH, 36:2 (1981), 11-80, Mi rm2891, MR 616797, Zbl 0478.58038.

[34] Н. И. Ахиезер, “Об ортогональных многочленах на нескольких интервалах", Докл. АН ССCP, 134:1 (1960), 9-12, zbl 0101.29205.

[35] Н.И. Ахиезер, Ю.Я. Томчук, “К теории ортогональных многочленов на нескольких интервалах", Докл. АН СССР, 138:4 (1961), 743-746, Zbl 0109.29601.

[36] Н. И. Ахиезер, “Континуальные аналоги ортогональных многочленов на системе интервалов", Докл. АН СССР, 141:2 (1961), 263266, Zbl 0109.29602.

[37] Б. М. Левитан, Обратные задачи Штурма-Лиувилля, Наука, М., 1984, MR 0771843.

[38] В. И. Арнольд, Математические методъ классической механики, Наука, М., 1989, MR 1037020.

[39] Б. А. Дубровин, "Периодические задачи для уравнения Кортевегаде Фриза в классе конечно-зонных потенциалов", Функи. анализ и его приложен., 9:3 (1975), 41-52, MR 486780.

[40] Г. М. Голузин, Геометрическая теория функиий комплексного переменного, Наука, М., 1966, MR 0219714.

[41] J.S. Jeronimo and W. Van Assche, "Orthogonal polynomials with asymptotically periodic recurrrence coefficients", J. Approx. Theory, 46 (1986), 251-283, doi 10.1016/0021-9045(86)90065-1, MR 840395. 
Научное издание

\section{Современные проблемы математики}

\section{Выпуск 6}

\section{Сергей Павлович Суетин \\ Сравнительная асимптотика решений и формулы следов для некоторого класса разностных уравнений}

Компьютерная верстка: B. М. Музафаров

Сдано в набор 15.06.2006. Подписано в печать 06.09.2006.

Формат $60 \times 90 / 16$. Усл. печ. л. 4,500. Тираж 200 экз.

Отпечатано в Математическом институте им. В. А. Стеклова РАН Москва, 119991, ул. Губкина, 8.

http://www.mi.ras.ru/spm/ e-mail: spm@mi.ras.ru 